\title{
A STUDY OF QUARK FRAGMENTATION USING KAONS PRODUCED IN ASSOCIATION WITH PROMPT $D_{S}^{ \pm} / D^{ \pm}$MESONS
}

\author{
A Dissertation \\ Submitted to the Faculty \\ of \\ Purdue University \\ by \\ Niharika Ranjan Singh \\ In Partial Fulfillment of the \\ Requirements for the Degree \\ of \\ Doctor of Philosophy
}

May 2012

Purdue University

West Lafayette, Indiana 
Dedicated to my parents, Vasudha and Awni Ranjan. 


\section{ACKNOWLEDGMENTS}

I would like to sincerely thank my adviser, Prof. Matthew Jones for his guidance, encouragement and patience throughout the course of my doctoral education. He has always inspired me to achieve the very best in my work and consistently supported me in good and difficult times alike.

I would like to thank the staff in the Physics department at Purdue University for making my years in graduate school comfortable and pleasant. I am also thankful to Prof. Daniela Bortoletto, Prof. Fuqiang Wang and Prof. Thomas E. Clark for agreeing to be on my Ph.D. committee.

I would also like to thank the Fermilab staff, the CDF collaboration and all the people who have contributed towards building the laboratory and the CDF detector. Without their effort the research presented in this dissertation would not have been possible. I am grateful to Dr. Guillelmo Gomez-Ceballos, Dr. Aart Heijboer, Dr. Azizur Rahaman and Dr. Gene Flanagan for helping me during my initial days, while I was based at Fermilab. They familiarized me with the work environment and jargon used at CDF which can be quite over-whelming for a beginner. I would also like to thank Prof. Robert Harr and Dr. Diego Tonelli for many insightful discussions and suggestions that helped in understanding the details and development of the analysis.

It would not have been possible for me to achieve any measure of success, in any realm of life, without the unconditional love and support of my family. My mother, Vasudha Ranjan, has always encouraged me to strive for the best and keeping a positive attitude. I still remember all the childhood days when she would wake up at wee hours in the morning and teach me the various subjects with utmost patience. I undoubtedly own my interest in Physics to my father, Awni Ranjan, who showed me how interesting and exciting science can be. I will always be immensely grateful to my parents for providing me with a very comfortable childhood and cultivating my 
attitude towards education and life in general. I would also like to thank my younger brother, Salleen Ranjan, for being such a fun, loving and supportive sibling.

I met my husband, Dr. Sarvjeet Singh, at Purdue University during the course of my graduate school. I am grateful to him for being immensely thoughtful and supportive during our time apart while I was completing my doctoral degree at Purdue. I feel extremely fortunate that I met and married such a wonderful person.

I would also like to thank my grandmother, Ahilya Devi Gautam, for her unconditional love. I can not express in words how grateful I am to her for everything she has done for me. Although born and raised in a patriarchal society, she has always supported me in all my decisions including my career choice - and for this I will always admire her. I would like to mention that my career choice has been greatly influenced by my late grandfather, Prof. Narendra Deo Gautam. I remember watching him work on his research with such perseverance and enthusiasm during my early childhood days. I wish that he would have been present to see me complete my doctoral degree.

I have also received lots of encouragement from my parents-in-law, Gurudev Singh and Parkash Kaur. I am thankful to them for their generous support and feel very happy that they are part of my family. Lastly but not the least, I would also like to thank my dear friends and relatives for their support and encouragement throughout the course of my doctoral education. 


\section{TABLE OF CONTENTS}

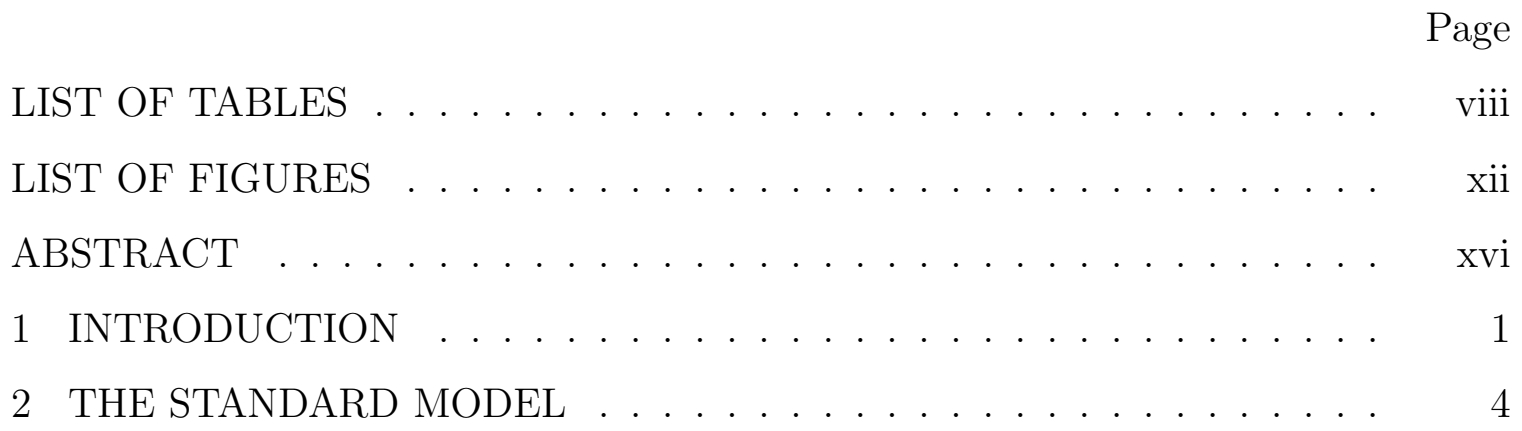

2.1 Quantum Chromodynamics . . . . . . . . . . . . . . 7

2.2 The Electroweak Theory . . . . . . . . . . . . . . . . . . . . . . . . . 9

2.2 .1 The Higgs Mechanism . . . . . . . . . . . . . . . . 11

2.2.2 The Cabibbo-Kobayashi-Maskawa (CKM) matrix . . . . . . 12

2.3 Summary . . . . . . . . . . . . . . . . . . . . . . 17

3 QUARK FRAGMENTATION . . . . . . . . . . . . . . . . . . 18

3.1 Initial parton shower . . . . . . . . . . . . . . . . . . . 18

3.2 Quark fragmentation . . . . . . . . . . . . . . . . . . . . 21

3.2 .1 The Feynman-Field Model . . . . . . . . . . . . . . . . 22

3.2 .2 The Lund String Model . . . . . . . . . . . . . . . . . . 24

3.2 .3 Peterson Fragmentation Model . . . . . . . . . . . . . . 26

3.2 .4 Cluster Fragmentation Model . . . . . . . . . . . . . . 28

3.3 Summary . . . . . . . . . . . . . . . . . . . . . . . . . . . . . . 29

4 EXPERIMENTAL APPARATUS . . . . . . . . . . . . . . . . 31

4.1 The Accelerator Complex . . . . . . . . . . . . . . . . 31

4.1.1 Pre-Accelerator . . . . . . . . . . . . . . . . 31

4.1 .2 Linear Accelerator . . . . . . . . . . . . . . . . . . . 32

4.1 .3 Booster . . . . . . . . . . . . . . . . . . . 33

4.1.4 Main Injector . . . . . . . . . . . . . . . . . . . . 34

4.1.5 Anti-Proton Production . . . . . . . . . . . . . . 34

4.1 .6 Tevatron . . . . . . . . . . . . . . . . 36

4.2 The Collider Detector at Fermilab . . . . . . . . . . . . 36

4.2.1 Conventions and definitions used at CDF . . . . . . . 37

4.2 .2 Tracking at CDF . . . . . . . . . . . . . . 39

4.2 .3 Silicon Detectors . . . . . . . . . . . . . . . 40

4.2 .4 Central Outer Tracker . . . . . . . . . . . . . . . . . . 41

4.2 .5 Time-of-Flight detector . . . . . . . . . . . . . . . 46

4.2 .6 Calorimeters and Muon Chambers . . . . . . . . . 47 
Page

4.2.7 Overview of the Trigger system at CDF . . . . . . . . . . 50

4.2.8 The two track trigger . . . . . . . . . . . . . . . . . 54

4.2 .9 Computing and Software . . . . . . . . . . . 58

5 PARTICLE IDENTIFICATION TECHNIQUES . . . . . . . . . . . . . . . 61

5.1 Particle identification using Time-of-Flight . . . . . . . . . . 61

5.1.1 TOF residual distribution . . . . . . . . . . . . . . 62

5.1.2 Calibrating the TOF residual distribution . . . . . . . . 63

5.2 Particle identification using $d E / d x$ measured in the COT . . . . . 68

5.2.1 Calibrating the COT $d E / d x$ response . . . . . . . . . . 69

5.3 Separation power of the particle identification techniques . . . . . . 72

5.4 Testing the combined particle identification techniques . . . . . . 78

5.4.1 Study using a pure sample of pions . . . . . . . . . . . . . 78

5.4.2 Study using a pure sample of kaons . . . . . . . . . . . . . . 79

5.4.3 Summary of particle identification test studies . . . . . . . . 81

5.5 Model to account for bias due to particle mis-identification . . . . . 81

5.5.1 Bias matrix elements obtained using a pion sample from $D^{*}$ decays ...................... . . . 82

5.5.2 Bias matrix elements obtained using a $\phi \rightarrow K^{+} K^{-}$sample . 83

5.5.3 Correcting for the bias in a generic sample . . . . . . . . . 84

5.6 Effect of kaons decaying in flight . . . . . . . . . . . . . . . . . . 84

5.7 Summary . . . . . . . . . . . . . . . . . . . 88

6 THE ANALYSIS TECHNIQUE . . . . . . . . . . . . . . . . . . . . 89

6.1 Identification of prompt $D_{s}^{ \pm} / D^{ \pm}$components in the sample . . . . . 90

6.1.1 Separation of $D_{s}^{ \pm} / D^{ \pm}$signal . . . . . . . . . . . . . . . . 90

6.1.2 Broad peak in the $K K \pi$ invariant mass distribution . . . . . 90

6.1.3 Likelihood fit using the $K K \pi$ invariant mass distribution . . 92

6.1.4 Separation of prompt and secondary $D_{s}^{ \pm} / D^{ \pm}$components . . 93

6.1.5 Shape of secondary $D_{s}^{ \pm} / D^{ \pm}$impact parameter distribution . 94

6.1.6 Fits to the $K K \pi$ invariant mass and impact parameter distributions......................... 99

6.2 Measurement of the kaon fraction around prompt $D_{s}^{ \pm} / D^{ \pm}$mesons . 104

6.2.1 Likelihood fitting procedure for measuring charged particle fractions .......................... 104

6.2.2 Kaon fraction extracted from the likelihood fitting procedure 107

7 OVERVIEW OF SYSTEMATIC STUDIES . . . . . . . . . . . . 110

7.1 Systematic uncertainty due to effect of kaons decaying in flight . . . 110

7.2 Systematic error due to mis-identification of particle types . . . . . 111

7.3 Systematic uncertainty due to TOF and $d E / d x$ parameterization . 112

7.4 Systematic uncertainty due to parametrization of $K K \pi$ mass and $d_{0}$ distributions . . . . . . . . . . . . . . . . 113

7.5 Summary of systematic studies . . . . . . . . . . . . . 115 
8 DISCUSSION OF RESULTS . . . . . . . . . . . . . . . . . . 117

8.1 Cross check using displaced tracks around secondary $D_{s}^{ \pm} \ldots \ldots$. . 136

9 SUMMARY . . . . . . . . . . . . . . . . . . . . . 139

LIST OF REFERENCES . . . . . . . . . . . . . . . . . . . 141

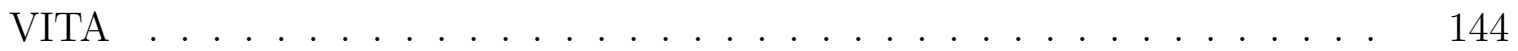




\section{LIST OF TABLES}

Table

Page

2.1 Properties of elementary particles described by the Standard Model theory. Each fermion has a corresponding anti-particle which has the same mass but opposite electric charge. . . . . . . . . . . . . . . . . .

2.2 An overview of the processes used for the determination of the CKM matrix elements. . . . . . . . . . . . . . . . . .

5.1 Particle fractions measured in the signal region of the pion sample from $D^{* \pm} \rightarrow D^{0} \pi^{ \pm}$decays. . . . . . . . . . . . . . . .

5.2 Particle fractions measured in the signal component of the $\phi \rightarrow K^{+} K^{-}$ sample. . . . . . . . . . . . . . . . . .

5.3 Probability that a kaon will be reconstructed track for different $p_{T}$ ranges. The table also lists the probability that a reconstructed kaon contributes to the measured kaon or pion fraction. . . . . . . . . . . . . .

5.4 Fraction of kaons reconstructed as charged tracks that are subsequently identified as pions. . . . . . . . . . . . . . . . .

5.5 Results of the linear fit performed on the distribution of fraction $w$ using the function $w\left(p_{T}\right)=a_{0}+a_{1} p_{T}$. The correlation coefficient is also listed in the table. . . . . . . . . . . . . . . . . . . . .

6.1 Yields of $D_{s}^{ \pm}$and $D^{ \pm}$mesons in ranges of transverse momentum $p_{T}$ of the reconstructed $K K \pi$ candidates. . . . . . . . . . . . . . . .

6.2 Yields of exclusive samples of B decays that were generated for studying the impact parameter distributions of secondary $D$ mesons. The table also lists the number of $K^{+} K^{-} \pi$ candidates that satisfy track quality criteria, offline selection cuts and SVT-like pair requirement with $7<$

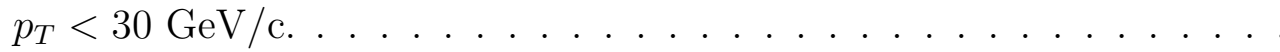

6.3 Values for prompt $D_{s}^{ \pm}$and prompt $D^{ \pm}$fraction in the data sample in ranges of transverse momentum $p_{T}$ of the reconstructed $K K \pi$ candidates. The systematic uncertainty in the prompt fraction listed in the table is calculated by propagating the statistical uncertainties in the secondary templates that are obtained using Monte Carlo samples of exclusive B decays. . . . . . . . . . . . . . . . . . 
7.1 List of the groups in which the parameters describing the various components/features in data are sorted. . . . . . . . . . . . . . . .

8.1 Measured kaon fraction around prompt $D_{s}^{ \pm}$and prompt $D^{ \pm}$mesons in ranges of transverse momentum $\left(p_{T}\right)$ of the maximum- $p_{T}$ track found in the cone, for the opposite sign charge combination. The first uncertainty is statistical and the second is the combination of all the systematic uncertainties. . . . . . . . . . . . . . . . .

8.2 Measured kaon fraction around prompt $D_{s}^{ \pm}$and prompt $D^{ \pm}$mesons in ranges of transverse momentum $\left(p_{T}\right)$ of the maximum- $p_{T}$ track found in the cone, for the same sign charge combination. The first uncertainty is statistical and the second is the combination of all the systematic uncertainties. . . . . . . . . . . . . . . . .

8.3 Measured kaon fraction $\left(f_{K}\right)$ around prompt $D_{s}^{ \pm}$and prompt $D^{ \pm}$mesons in ranges of transverse momentum $\left(p_{T}\right)$ of the maximum- $p_{T}$ track found in the cone, for the opposite sign charge combination. The first uncertainty is the statistical error (stat) followed by the systematic uncertainties due to sources such as: Effect of kaons decaying in flight (DIF), probability density functions used to separate prompt and secondary D components (PDF), calibration of Time-of-Flight and $d E / d x$ distributions for particle identification (calib) and mis-identification of particle species (mis-ID).

8.4 Measured kaon fraction $\left(f_{K}\right)$ around prompt $D_{s}^{ \pm}$and prompt $D^{ \pm}$mesons in ranges of transverse momentum $\left(p_{T}\right)$ of the maximum- $p_{T}$ track found in the cone, for the same sign charge combination. The first uncertainty is the statistical error (stat) followed by the systematic uncertainties due to sources such as: Effect of kaons decaying in flight (DIF), probability density functions used to separate prompt and secondary D components (PDF), calibration of Time-of-Flight and $d E / d x$ distributions for particle identification (calib) and mis-identification of particle species (mis-ID).

8.5 Measured kaon fraction around prompt $D_{s}^{ \pm}$and prompt $D^{ \pm}$mesons in ranges of the invariant mass of the $\mathrm{D}$ candidate and the maximum- $p_{T}$ track found in the cone $\left(m_{D K}\right)$, for the opposite sign charge combination. The first uncertainty is statistical and the second is the combination of all the systematic uncertainties. . . . . . . . . . . . . .

8.6 Measured kaon fraction around prompt $D_{s}^{ \pm}$and prompt $D^{ \pm}$mesons in ranges of the invariant mass of the $\mathrm{D}$ candidate and the maximum- $p_{T}$ track found in the cone $\left(m_{D K}\right)$, for the same sign charge combination. The first uncertainty is statistical and the second is the combination of all the systematic uncertainties. . . . . . . . . . . . . . . . 
8.7 Measured kaon fraction $\left(f_{K}\right)$ around prompt $D_{s}^{ \pm}$and prompt $D^{ \pm}$mesons in ranges of the invariant mass of the $\mathrm{D}$ candidate and the maximum- $p_{T}$ track found in the cone $\left(m_{D K}\right)$, for the opposite sign charge combination. The first uncertainty is the statistical error (stat) followed by the systematic uncertainties due to sources such as: Effect of kaons decaying in flight (DIF), probability density functions used to separate prompt and secondary D components (PDF), calibration of Time-of-Flight and $d E / d x$ distributions for particle identification (calib) and mis-identification of particle species (mis-ID) . . . . . . . . . . . . . . .

8.8 Measured kaon fraction $\left(f_{K}\right)$ around prompt $D_{s}^{ \pm}$and prompt $D^{ \pm}$mesons in ranges of the invariant mass of the $\mathrm{D}$ candidate and the maximum- $p_{T}$ track found in the cone $\left(m_{D K}\right)$, for the same sign charge combination. The first uncertainty is the statistical error (stat) followed by the systematic uncertainties due to sources such as: Effect of kaons decaying in flight (DIF), probability density functions used to separate prompt and secondary D components (PDF), calibration of Time-of-Flight and $d E / d x$ distributions for particle identification (calib) and mis-identification of particle species (mis-ID) . . . . . . . . . . . . . . . .

8.9 Measured kaon fraction around prompt $D_{s}^{ \pm}$and prompt $D^{ \pm}$mesons in ranges of the rapidity difference between the $\mathrm{D}$ candidate and the maximum$p_{T}$ track found in the cone along the direction of the $\mathrm{D}$ momentum axis $(\Delta y)$, for the opposite sign charge combination. The first uncertainty is statistical and the second is the combination of all the systematic uncertainties. . . . . . . . . . . . . . . . .

8.10 Measured kaon fraction around prompt $D_{s}^{ \pm}$and prompt $D^{ \pm}$mesons in ranges of the rapidity difference between the $\mathrm{D}$ candidate and the maximum$p_{T}$ track found in the cone along the direction of the $\mathrm{D}$ momentum axis $(\Delta y)$, for the same sign charge combination. The first uncertainty is statistical and the second is the combination of all the systematic uncertainties.

8.11 Measured kaon fraction $\left(f_{K}\right)$ around prompt $D_{s}^{ \pm}$and prompt $D^{ \pm}$mesons in ranges of the rapidity difference between the $\mathrm{D}$ candidate and the maximum- $p_{T}$ track found in the cone along the direction of the $\mathrm{D}$ momentum axis $(\Delta y)$, for the opposite sign charge combination. The first uncertainty is the statistical error (stat) followed by the systematic uncertainties due to sources such as: Effect of kaons decaying in flight (DIF), probability density functions used to separate prompt and secondary D components (PDF), calibration of Time-of-Flight and $d E / d x$ distributions for particle identification (calib) and mis-identification of particle species (mis-ID). . . . . . . . . . . . . . . . . 
8.12 Measured kaon fraction $\left(f_{K}\right)$ around prompt $D_{s}^{ \pm}$and prompt $D^{ \pm}$mesons in ranges of the rapidity difference between the $\mathrm{D}$ candidate and the maximum- $p_{T}$ track found in the cone along the direction of the $\mathrm{D}$ momentum axis $(\Delta y)$, for the same sign charge combination. The first uncertainty is the statistical error (stat) followed by the systematic uncertainties due to sources such as: Effect of kaons decaying in flight (DIF), probability density functions used to separate prompt and secondary D components (PDF), calibration of Time-of-Flight and $d E / d x$ distributions for particle identification (calib) and mis-identification of particle species (mis-ID).

8.13 Measured kaon fraction in a sample of displaced tracks found in a cone around secondary $D_{s}^{ \pm}$mesons. The table also shows predictions of the PYTHIA event generator. . . . . . . . . . . . . . . 


\section{LIST OF FIGURES}

Figure

Page

1.1 Naive picture of the quark fragmentation process depicting the production of lighter bound states such as kaons $K^{ \pm}$and pions $\pi^{ \pm}$in association with heavier bound states such as $D_{s}^{ \pm}$and $D^{ \pm}$via the combination of charm $c$ quarks with lighter quarks such as down $d$ and strange $s$ quarks. . . . .

2.1 A sketch of the unitary triangle resulting from the relation in Equation $2.20 \ldots \ldots \ldots \ldots \ldots \ldots$

3.1 Schematic representation of the production of bottom quarks in a $p \bar{p}$ interaction, which subsequently fragment to form b-hadrons that undergo strong and then weak decays to lighter mesons. . . . . . . . . . . .

3.2 Feynman diagrams for some leading order QCD processes resulting in the creation of heavy quark-antiquark pairs $Q \bar{Q}$. . . . . . . . . . . .

3.3 Feynman diagrams for some higher order QCD processes resulting in the

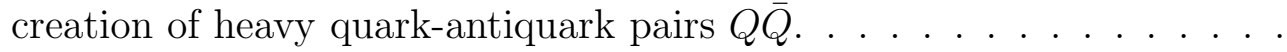

3.4 Lines of force of the gluon field between a $q_{1} \bar{q}_{1}$ pair created in a high energy interaction. The gluon flux lines fragment as $q_{1}$ and $\bar{q}_{1}$ move apart, creating quark antiquark pair $q_{2} \bar{q}_{2}$ at the breaking point. . . . . . . . .

3.5 The fragmentation of a heavy quark $Q$ into a meson $H(Q \bar{q})[14]$. . . .

3.6 Fragmentation functions $D_{c}(z)$ and $D_{b}(z)$ for charm and bottom quarks. The functional form is shown in Equation 3.17. The distributions are made using $\epsilon_{c}=0.15$ and $\epsilon_{b}=0.15\left(m_{c} / m_{b}\right)^{2}=0.016$ [14] . . . . . . .

3.7 Example diagram showing evolution of jets using cluster fragmentation model, in a typical high energy interaction. Final partons $q_{v}, \overline{q_{1}} \ldots \overline{q_{v}}$ have energy of the order of few $\mathrm{GeV}$ and combine to form color singlet clusters

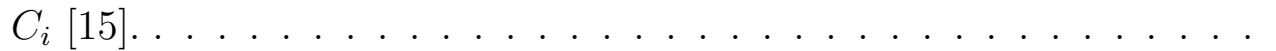

4.1 Overview of the accelerator complex at the Fermi National Accelerator Laboratory (Fermilab) [16]. . . . . . . . . . . . . . . 32

4.2 An isometric view of the Collider Detector at Fermilab [19]. . . . . . .

4.3 A schematic view showing the passage and detection of different particles in the various sub-systems of CDF II [19]. . . . . . . . . . . . . . 
4.4 An overview of the tracking layout at CDF II showing the different subdetector systems [19]. . . . . . . . . . . . . .

4.5 A cross-sectional view of the silicon detector at CDF II showing the three sub-detector systems in the $r-\phi$ plane $[19] \ldots . . . . . . . .$.

4.6 A cross-sectional view of the silicon detector at CDF II showing the three

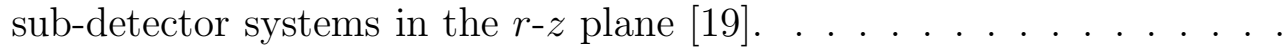

4.7 Layout of the field sheet and sense wire planes in $1 / 6$ th section of the

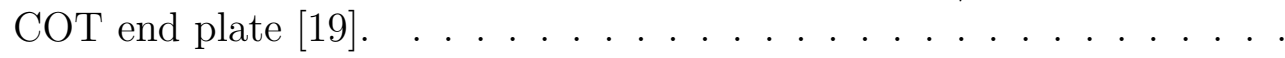

4.8 Layout of wires in three supercells in $2^{\text {nd }}$ superlayer of the COT which

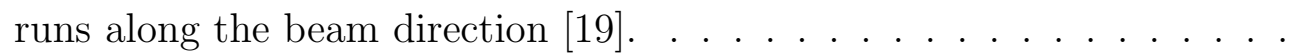

4.9 Location of the Time-of-Flight system in the Collider Detector at Fermilab $[19] \ldots \ldots \ldots \ldots \ldots \ldots$

4.10 A schematic diagram of the CDF II trigger system. The $p \bar{p}$ bunch crossing interval is $396 \mathrm{~ns}$ although the Tevatron and CDF II are designed to handle a bunch crossing interval of $132 \mathrm{~ns}$. The nominal data storage frequency is $100 \mathrm{~Hz}[19] \ldots \ldots \ldots \ldots \ldots$

4.11 Block diagram illustrating the flow of information from the CDF II detector elements through the different components of the trigger system [19].

5.1 (1) Invariant mass difference distribution, $\Delta m=m(K \pi \pi)-m(K \pi)$, for the reconstructed $K \pi \pi$ candidates (2) TOF residual distribution for the pion track, assuming the pion mass hypothesis. The data corresponds to a momentum range of $0.4<p_{T}(\pi)<0.45 \mathrm{GeV} /$ c. . . . . . . . . . . . .

5.2 Energy loss per unit length $d E / d x$ for various particles traversing a drift chamber: (a) Response of a drift chamber filled with gas at a pressure of 8.5 atm, optimized for $d E / d x$. (b) Response of the CDF-II drift chamber that is not particularly optimized for $d E / d x$ measurements. . . . . .

$5.3 d E / d x$ distribution in data and projections of the parameterization used to describe the shape of the universal curve. . . . . . . . . . . . . .

$5.4 Z(d E / d x)$ distribution and projections from the likelihood fit, plotted assuming the pion mass hypothesis. . . . . . . . . . . .

5.5 Measured separation power of the particle identification technique using Time-of-Flight. . . . . . . . . . . . . . . . . 76

5.6 Measured separation power of the particle identification using $d E / d x$ for positively and negatively charged tracks. . . . . . . . . . . . 
$5.7 p_{T}$ distribution of fraction of reconstructed kaons subsequently identified as pions. . . . . . . . . . . . . . . . . . . 88

$6.1 K K \pi$ invariant mass distribution in data. . . . . . . . . . . . . . . 91

$6.2 K K \pi$ invariant mass distribution of mis-reconstructed $D^{+} \rightarrow K^{-} \pi^{-} \pi^{+}$ decays in Monte Carlo events. . . . . . . . . . . . . . . . . .

6.3 $K K \pi$ invariant mass distribution in data and projections from the likelihood fit for $7<p_{T}<8 \mathrm{GeV} /$ c. . . . . . . . . . . . .

6.4 A secondary $D$ meson can have non zero impact parameter $\left(d_{0}\right)$ with respect to the primary vertex, whereas, a prompt $D$ meson will point back to the primary vertex and should ideally have zero impact parameter. .

6.5 Impact parameter distribution of $K K \pi$ candidates found in Monte Carlo samples of B decays, superimposed with projections obtained from the likelihood fit.

6.6 Impact parameter distribution of the $K K \pi$ candidates in the $D_{s}^{ \pm} / D^{ \pm}$ signal region and projections from the likelihood fit showing the prompt, secondary and background components. . . . . . . . . . . . .

6.7 The fraction of kaons in the sample of maximum- $p_{T}$ tracks found in a cone around $D_{s}^{ \pm}$mesons in events generated using PYTHIA. The $p_{T}$ distribution is plotted for PYTHIA samples generated by including and excluding the tuning of the underlying event in the simulation. As we can see in the plot, the tuning does not affect the $p_{T}$ distribution of the maximum- $p_{T}$ tracks found in the cone. The two tunes use different sets of parameters to describe the underlying event. . . . . . . . . . . . . . . .

6.8 Distribution of kaon fraction measured around prompt $D_{s}^{ \pm} / D^{ \pm}$mesons in ranges of transverse momentum $\left(p_{T}\right)$ of the maximum- $p_{T}$ track found in the cone. The error bars depict only statistical uncertainty obtained from the likelihood fit. . . . . . . . . . . . . . . . . .

8.1 Kaon fraction measured around prompt $D_{s}^{ \pm} / D^{ \pm}$mesons in ranges of transverse momentum of the maximum- $p_{T}$ track found in the cone. . . . . .

8.2 Kaon fraction measured around prompt $D_{s}^{ \pm} / D^{ \pm}$mesons in ranges of invariant mass of the the maximum- $p_{T}$ track in the cone and the $D$ candidate. In graph $(a)$, the kaon fraction peaks around $m_{D K}=2.46 \mathrm{GeV} / \mathrm{c}^{2}$, which corresponds to correct mass hypothesis in cases where the maximum$p_{T}$ track is likely to be an opposite sign kaon produced in the second branch. . . . . . . . . . . . . . . . . . 
Figure

8.3 Kaon fraction measured around prompt $D_{s}^{ \pm} / D^{ \pm}$mesons in ranges of rapidity difference between the maximum- $p_{T}$ track and the $D$ candidate along the $D$ momentum axis. In graph $(a)$, the kaon fraction in the opposite sign combination peaks around $\Delta y=0$, which implies that the maximum- $p_{T}$ track with almost similar rapidity as compared to the $D_{s}^{ \pm}$meson is more likely to be a kaon. . . . . . . . . . . . . . . . . .

8.4 Distribution of kaon fraction measured around secondary $D_{s}^{ \pm}$mesons as a function of the transverse momentum of the maximum- $p_{T}$ displaced track found in the cone. . . . . . . . . . . . . . . . . . . . . 


\begin{abstract}
Singh, Niharika Ranjan. Ph.D., Purdue University, May 2012. A Study of Quark Fragmentation using Kaons Produced in Association with Prompt $D_{s}^{ \pm} / D^{ \pm}$Mesons. Major Professor: Matthew Jones.
\end{abstract}

Quarks are considered to be the fundamental constituents of hadronic matter, but they have never been observed as free particles. When quarks are produced at high energy colliders, they quickly form bound colorless states, which then decay to produce the particles observed in experiments. The process by which an initially free quark combines with other quarks to form a hadronic particle is called quark fragmentation and has been described using phenomenological models since quarks were first proposed. Since then, several models have been developed to describe the quark fragmentation phenomenon, and these have been tuned to reproduce many average properties of hadrons produced in high energy collisions.

In this dissertation, we describe an analysis that probes the properties of particles produced in association with a hadron containing a charm quark that provides a way, for the first time, to study what is thought of as the second particle produced in the process of heavy quark fragmentation. Data from proton anti-proton collisions was used to carry out this research, which were collected with the CDF II detector at the Fermilab Tevatron and corresponds to $360 p b^{-1}$ of integrated luminosity. We reconstruct $D_{s}^{ \pm}$and $D^{ \pm}$mesons, which contain charm quarks, and identify the kaons produced in association with them. The kinematic properties of these kaons are compared with predictions of the fragmentation models implemented in the PYTHIA and HERWIG event generators. We find that kaon production in association with $D_{s}^{ \pm}$ mesons is enhanced at levels that are in agreement with the fragmentation models but observe differences in production rates of kaons that are produced later in the fragmentation process. 


\section{INTRODUCTION}

The charm quark was discovered almost 40 years via the observation of the $J / \psi$ particle that is a bound state containing a charm quark/anti-quark pair. This discovery was significant because bound excited states of the charm quarks emitted photons with regular energy spacing. Hence, precision spectroscopy of the two body charmonium system provided a good method to probe the physics of the strong interaction, similar to the case of the Hydrogen where studying atomic transitions significantly contributed to the understanding of the electromagnetic (EM) interaction.

It was discovered that the coupling constant that determines the strength of the strong interaction varies with separation or momentum transfer between quarks. In the limit of infinitely small separation or larger momentum transfer, the strong interaction becomes weaker and the quarks are nearly free at very short distances. This phenomenon is known as asymptotic freedom. Conversely, as the separation between quarks increases (order $\sim 1 \mathrm{fm}$ ), the strength of the strong interaction increases leading to quark confinement leading to the impossibility of observing free quarks.

Perturbation theory can be used to describe the properties of quarks at small distances or high momentum transfers but it fails to describe the properties of low momentum phenomenon. Hence, we mainly rely on models to emulate the features of low momentum phenomena. An example of such a process is the quark fragmentation phenomena by which quarks combine to form bound states.

Since the description of the fragmentation process is provided using models, it is interesting to check these models with measurements in data. A unique way to probe the quark fragmentation phenomenon is to study the production of charged bound states containing lighter quarks in association with production of bound states containing heavier quarks. In the analysis presented in this dissertation, we study the production of charged kaons (bound states of up and strange quarks) in association 
with the production of $D_{s}^{ \pm}$(bound state of charm and strange quarks) and $D^{ \pm}$(bound state of charm and down quarks) mesons. A naive picture of the $D_{s}^{ \pm} / D^{ \pm}$production via the fragmentation process is shown in Figure 1.1. As illustrated in the figure, a kaon with opposite sign charge is produced in the second branch of the fragmentation process when a $D_{s}^{ \pm}$is produced, whereas a pion is produced when a $D^{ \pm}$meson is created. Kaons with same sign charge are likely to be produced at similar rates around both $D_{s}^{ \pm}$and $D^{ \pm}$mesons as shown in the figure. Hence by studying the properties of kaons produced in association with $D_{s}^{ \pm} / D^{ \pm}$mesons we can extract information about the various stages of the fragmentation phenomenon. Studying the production of opposite sign kaons around $D_{s}^{ \pm}$mesons can provide information specifically about the second branch in the fragmentation process. Information about distribution of kaons produced in subsequent branches can be extracted by studying properties of same sign kaons around both $D_{s}^{ \pm} / D^{ \pm}$mesons.
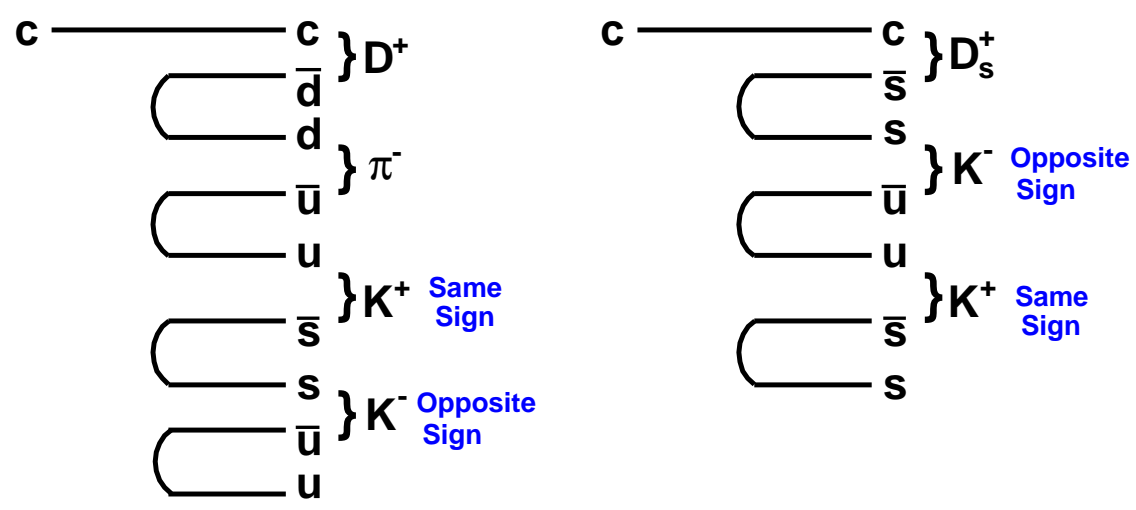

Figure 1.1.: Naive picture of the quark fragmentation process depicting the production of lighter bound states such as kaons $K^{ \pm}$and pions $\pi^{ \pm}$in association with heavier bound states such as $D_{s}^{ \pm}$and $D^{ \pm}$via the combination of charm $c$ quarks with lighter quarks such as down $d$ and strange $s$ quarks. 
The outline of rest this dissertation is as follows. In Chapter 2, we discuss the Standard Model in particle physics that is a theory describing the elementary particles and their interactions via the fundamental forces in nature. A brief overview of the models used to describe the quark fragmentation phenomena is presented in Chapter 3. We use particle identification techniques to identify lighter charged bound states such as kaons and pions using the techniques described in Chapter 5. The overview analysis technique used to extract the distributions of kaons produced in association with $D_{s}^{ \pm}$and $D^{ \pm}$mesons is described in Chapter 6. We study various sources that can introduce systematic uncertainties in the measurements, which are described in Chapter 7. Chapter 8 provides a discussion of the results of the charm quark fragmentation analysis. A summary of the analysis and the results is provided in Chapter 9. 


\section{THE STANDARD MODEL}

The Standard Model in particle physics attempts to describe three of the four known fundamental forces in nature and the properties of the elementary particles that interact via these forces, which are listed in Table 2.1. The fundamental forces that are described extremely well by the Standard Model theory are the electromagnetic, the weak and the strong force. The fourth fundamental force, gravity, is very weak at the energies currently accessible in particle physics experiments and is excluded from the Standard Model. The elementary particles described by the theory include quarks and leptons, which constitute all the atomic matter that comprises about $4.6 \%$ of the universe, and the force carriers that are mediators of the fundamental forces. About $23 \%$ of the universe is composed of dark matter, which may have a small proportion, known as baryonic dark matter, that is composed of quarks and leptons.

The quarks and leptons are fermions with spin 1/2, whereas the force carrier particles are bosons with spin one. Table 2.1 lists some properties of the 12 elementary fermions and the force mediating bosons [1]. Each fermion has a corresponding antiparticle, which has the same mass but opposite electric charge. Both leptons and quarks can experience the weak interaction mediated via $W^{ \pm}$and $Z$ bosons. The electromagnetic (EM), force mediated via photons, is only experienced by charged particles, and therefore, it affects quarks and charged leptons but not neutrinos. The strong force, mediated via gluons, is only experienced by quarks since leptons do not posses a color charge. The concept of color charge is similar to the concept of electric charge described in the EM interaction, but is fundamentally distinct in principle. Unlike the electric charge that can only take one value, namely positive (or negative) charge, the color charge can take either of the three values, red (or anti-red), blue (or anti-blue) and green (or anti-green). Bound states of quarks are known as hadrons and can undergo the strong, weak and electromagnetic interactions. The hadrons are 
Table 2.1: Properties of elementary particles described by the Standard Model theory. Each fermion has a corresponding anti-particle which has the same mass but opposite electric charge.

\begin{tabular}{|c|c|c|c|}
\hline & Particle & Mass & Charge \\
\hline \multirow{6}{*}{$\begin{array}{l}\text { Quarks } \\
\text { (Fermions) }\end{array}$} & $\operatorname{up}(u)$ & $1.7-3.3 \mathrm{MeV} / c^{2}$ & $+2 / 3$ \\
\hline & down $(d)$ & $4.1-5.8 \mathrm{MeV} / c^{2}$ & $-1 / 3$ \\
\hline & $\operatorname{charm}(c)$ & $1.27_{-0.09}^{+0.07} \mathrm{GeV} / c^{2}$ & $+2 / 3$ \\
\hline & strange $(s)$ & $101_{-21}^{+29} \mathrm{MeV} / c^{2}$ & $-1 / 3$ \\
\hline & top $(t)$ & $172.0 \pm 0.9 \pm 1.3 \mathrm{GeV} / c^{2}$ & $+2 / 3$ \\
\hline & bottom $(b)$ & $4.19_{-0.06}^{+0.18} \mathrm{GeV} / c^{2}$ & $-1 / 3$ \\
\hline \multirow{6}{*}{$\begin{array}{l}\text { Leptons } \\
\text { (Fermions) }\end{array}$} & electron $(e)$ & $0.511 \mathrm{MeV} / c^{2}$ & -1 \\
\hline & electron-neutrino $\left(\nu_{e}\right)$ & $<2 \mathrm{eV} / c^{2}$ & 0 \\
\hline & muon $(\mu)$ & $105.66 \mathrm{MeV} / c^{2}$ & -1 \\
\hline & muon-neutrino $\left(\nu_{\mu}\right)$ & $<0.19 \mathrm{MeV} / c^{2}$ & 0 \\
\hline & $\operatorname{tau}(\tau)$ & $1.78 \mathrm{GeV} / c^{2}$ & -1 \\
\hline & tau-neutrino $\left(\nu_{\tau}\right)$ & $<18.2 \mathrm{MeV} / c^{2}$ & 0 \\
\hline \multirow{4}{*}{$\begin{array}{l}\text { Force Carriers } \\
\text { (Bosons) }\end{array}$} & gluon $(g)$ & 0 & 0 \\
\hline & photon $(\gamma)$ & $<1 \times 10^{-18} \mathrm{eV}$ & 0 \\
\hline & $W^{ \pm}$ & $80.40 \pm 0.02 \mathrm{GeV} / c^{2}$ & \pm 1 \\
\hline & $Z^{0}$ & $91.188 \pm 0.002 \mathrm{GeV} / c^{2}$ & 0 \\
\hline
\end{tabular}

further classified into baryons, which are made up of three quarks, and mesons, which are comprised of a quark anti-quark pair.

The fundamental laws of nature do not seem to change if the fermions are relabeled in different ways. For example, the three color charges carried by quarks can be re-defined independently at each point in space without changing any physical observables. This freedom is an example of a symmetry transformation under which 
the theory is invariant, which is an idea central to the construction of the Standard Model.

The mathematical formulation of the Standard Model is described by a nonabelian gauge theory with the $S U(3) \times S U(2) \times U(1)$ symmetry group, and is based on relativistic quantum field theory. In a gauge field theory, fields are represented by quantum mechanical operators that are used to describe particles such as quarks, leptons and force carriers. Quantum Chromodynamics (QCD) forms the $S U(3)$ component that describes the strong interaction, and the $S U(2) \times U(1)$ component forms the electroweak theory, which is a unified theory describing both the weak and the electromagnetic interactions.

As an example of a gauge field theory, a brief description of the gauge symmetry in the theory of Quantum Electrodynamics (QED) is provided below, which was the first gauge theory to be formulated [2]. QED describes the electromagnetic interaction and the Lagrangian is given by

$$
\mathcal{L}=\bar{\psi}\left[i D_{\mu} \gamma_{\mu}-m\right] \psi-\frac{1}{4} F_{\mu \nu} F_{\mu \nu}
$$

where $D_{\mu}$ is the covariant derivative given by $D_{\mu}=\partial_{\mu}-i e A_{\mu}$, and $F_{\mu \nu}=\partial_{\mu} A_{\nu}-\partial_{\nu} A_{\mu}$ is the operator of the electromagnetic field strength. The notations $m$ and $e$ represent the mass of an electron and the electric charge, $\gamma_{\mu}$ are four Dirac matrices. The operators $A_{\mu}(x)$ and $\psi(x)$ represent the photon field and the positron $\left(e^{+}\right)$fields respectively. The electron field is given by $\bar{\psi}(x)$.

Since QED is a local gauge theory, the Lagrangian is invariant under the following local gauge transformation of the field operators:

$$
\begin{gathered}
\psi(x) \rightarrow e^{i e \Lambda(x)} \psi(x) \\
\bar{\psi}(x) \rightarrow \bar{\psi}(x) e^{-i e \Lambda(x)} \\
A_{\mu}(x) \rightarrow A_{\mu}(x)+\partial_{\mu} \Lambda(x)
\end{gathered}
$$

where $\Lambda(x)$ is an arbitrary real valued function of space and time. Other charged fermion fields, such as those representing quarks transform similarly to $\psi(x)$. The 
phase factors $e^{i e \Lambda(x)}$ belong to the symmetry group of unitary transformations in one dimension. The general principles used in QED are extended to the other fundamental forces by exploring other possible symmetry groups, such as the $S U(3)$ group in Quantum Chromodynamics, which is briefly described below.

\subsection{Quantum Chromodynamics}

QCD is the theory of the strong color interactions between quarks and is based on the postulate that the $S U(3)$ color symmetry is locally gauge invariant. The color charge in the strong interactions is analogous to the electric charge in the electromagnetic interactions and both the strong and electromagnetic forces are mediated by massless gauge bosons. However, gauge invariance in QCD is more complicated than in QED and requires eight gauge bosons, namely the gluons, which also carry color charge. This in contrast to QED, which has only one gauge boson, namely the photon, which is electrically neutral. In addition, the local gauge invariance of the QCD Lagrangian requires terms that result in self-coupling of the gluon field octet. The gluon field operator $A_{\mu}$ is given by a matrix in color space:

$$
A_{\mu}=\sum_{i} A_{\mu}^{i} \lambda_{i} / 2
$$

where $A_{\mu}^{i}$ are field operators of eight gluon fields, $\lambda_{i}$ are the eight Gell-Mann matrices and $g_{s}$ is the strong coupling constant. In QCD, the covariant derivative takes the form:

$$
D_{\mu}=\partial_{\mu}-i g_{s} A_{\mu}
$$

In case of gluons, the field tensor $F_{\mu \nu}$ is expressed through $A_{\mu}$ by a more complex form than in the case of the photons:

$$
F_{\mu \nu}=\partial_{\mu} A_{\nu}-\partial_{\nu} A_{\mu}-i g_{s}\left[A_{\mu} A_{\nu}-A_{\nu} A_{\mu}\right]
$$

which is different from the operator of the photon field shown in Equation 2.1 and contains terms that result in self-coupling of the gluon fields, which represents the 
self-interaction of gluons. The eight colored gluon fields can be represented as combinations of the red $(r)$, blue $(b)$ and green $(g)$ color and anti-color states [3]: Quarks $(q)$ can have either of the three $r, b, g$ color (or anti-color) charges and the gluons carry pairs of color charges such as $r \bar{b}, b \bar{g}$. As a consequence of the self-coupling between gluons, the strong force allows quarks to form bound hadronic states only in colorless $q q q$ or $q \bar{q}$ combinations of quarks. Other combinations such as $q q$ or $q q \bar{q} \bar{q}$ do not form bound states since the strong interaction is found to be repulsive.

Another important consequence of the self-coupling of gluons is that the strength of the strong interaction between quarks diminishes at small distances, a phenomenon known as asymptotic freedom. At short distances $(r \ll 1 \mathrm{fm})$, the color potential between quarks is like a Coulomb potential and varies as $\sim 1 / r$. The running coupling constant $\alpha_{s}(r)=g_{s}^{2}(r) / 4 \pi$ decreases at very small distances where the momentum transfer $q$ increases, and at sufficiently large momentum transfer, the first order perturbative expression for $\alpha_{s}$ is given by:

$$
\alpha_{s}(q) \approx \frac{2 \pi}{b \ln (q / \Lambda)}
$$

where $b=11-(2 / 3) n_{f}$ depends on the number of quark flavors $n_{f}$ and the constant $\Lambda$ is of the order of $0.1 \mathrm{GeV}$. Conversely, as the separation between quarks increases to the order of $r \sim 1 \mathrm{fm}$, the strength of the strong interaction increases, leading to quark confinement which prohibits the existence of free quarks. At distances of $r \sim 1 \mathrm{fm}$, the strong coupling constant becomes large and perturbative QCD calculations do not converge rapidly. Instead, at such large distances or low momentum regimes, the description of the interactions between quarks is provided using various phenomenological models. One important low momentum phenomena that is of primary interest in this dissertation, is referred to as quark fragmentation, which is discussed in Chapter 3. 


\subsection{The Electroweak Theory}

The electroweak theory differs from QED and QCD in two characteristic features. Firstly, the gauge theory is spontaneously broken resulting in massive gauge bosons as discussed in Section 2.2.1. Secondly, the theory violates parity [2]. The parity violation is exhibited by the fact that $W^{ \pm}$bosons, which mediate the charged weak interactions, only couple to left handed fermions that have spins aligned opposite to the direction of the momentum vector. On the other hand, the $Z^{0}$ bosons, which mediate the neutral weak interactions, couple to both left handed and right handed fermions, but with different coupling strength. Furthermore, we have no experimental evidence of the existence of right handed neutrinos or left handed anti-neutrinos, which are absent in the Standard Model. This is based on the fact that processes like $\beta$ decay are observed to involve only left handed neutrinos.

The eigenstates of fermion fields in the electroweak theory are classified into left handed doublets and right handed singlets as follows:

$$
\begin{gathered}
\left(\begin{array}{l}
u \\
d^{\prime}
\end{array}\right)_{L}\left(\begin{array}{l}
c \\
s^{\prime}
\end{array}\right)_{L}\left(\begin{array}{l}
t \\
b^{\prime}
\end{array}\right)_{L} \\
\left(\begin{array}{l}
e \\
\nu_{e}
\end{array}\right)_{L}\left(\begin{array}{l}
\mu^{-} \\
\nu_{\mu}
\end{array}\right)_{L}\left(\begin{array}{l}
\tau^{-} \\
\nu_{\tau}
\end{array}\right)_{L} \\
(u)_{R}(c)_{R}(t)_{R} \\
(d)_{R}(s)_{R}(b)_{R} \\
\left(e^{-}\right)_{R}\left(\mu^{-}\right)_{R}\left(\tau^{-}\right)_{R}
\end{gathered}
$$

The formalism of the unified electroweak gauge theory is given by the $S U(2) \times U(1)$ component in the Standard Model. The symmetry in the $S U(2)$ group refers to the symmetry of the left-handed doublets in Equation 2.9. The gauge symmetry in the 
$U(1)$ component is similar to but distinct from that of QED. Local gauge invariance in the $S U(2)$ group requires three gauge bosons, two of which correspond to the $W^{+} / W^{-}$bosons. These charged gauge bosons are responsible for the dominant flavor changing electroweak transitions between the three generations of leptons/quarks. The local gauge invariance in the $U(1)$ symmetry group requires another gauge boson field, referred to as $B^{0}$, which mixes with the third gauge boson $W^{0}$ from the $S U(2)$ symmetry group. The mixing of the gauge boson fields corresponding to $B^{0}$ and $W^{0}$ produce the physical fields corresponding to the $Z^{0}$ boson and the photon $(\gamma)$. The gauge boson fields and the physical fields are related by the following transformation:

$$
\left(\begin{array}{c}
\gamma \\
Z^{0}
\end{array}\right)=\left(\begin{array}{cc}
\cos \theta_{W} & \sin \theta_{W} \\
-\sin \theta_{W} & \cos \theta_{W}
\end{array}\right)\left(\begin{array}{c}
B^{0} \\
W^{0}
\end{array}\right)
$$

where $\theta_{W}$ is the weak mixing angle.

The gauge symmetries in the Standard Model appear to be fundamental nature and moreover, they provide a theory that is renormalizable. In a renormalizable theory, infinities arising in the calculations of physical observables can be absorbed into the definition of masses, coupling constants or other parameters of the theory that must be determined experimentally. According to the theoretical predictions, the $W^{ \pm}$and $Z^{0}$ bosons should be massless like the photon. However, experimental results showed that the $W^{ \pm}$and $Z^{0}$ bosons are actually extremely massive. To overcome this discrepancy between theoretical predictions and experimental results, the $S U(2) \times$ $U(1)$ gauge symmetry is spontaneously broken via the Higgs mechanism discussed in the section below. 


\subsubsection{The Higgs Mechanism}

In the unbroken electroweak theory, the massless gauge bosons consist of a weakisospin triplet, $\vec{W}$, and an isospin singlet $B$. The Higgs mechanism introduces an additional weak isospin doublet of scalar Higgs fields that is denoted by:

$$
\Phi=\left(\begin{array}{c}
\phi^{+} \\
\phi^{0}
\end{array}\right)
$$

A renormalizable form for the scalar potential $V$ is given by $[3,4]$ :

$$
V=\frac{1}{2} \mu^{2}|\Phi|^{2}+\frac{1}{4} \lambda|\Phi|^{4}
$$

where $\mu$ is the particle mass and $\lambda$ is a dimensionless coupling constant. The minimum value of $V$ occurs when $\partial V / \partial \Phi=0$ i.e.

$$
\Phi\left(\mu^{2}+\lambda \Phi^{2}\right)=0
$$

If $\mu^{2}>0$, the lowest energy vacuum state with $V=0$ is when $\Phi=0$. However, if $\mu^{2}<0$ then the lowest energy state is given by $V=-\mu^{4} / 4 \lambda$ at $\Phi= \pm \sqrt{\frac{-\mu^{2}}{\lambda}}$. The quantity $v=\sqrt{\frac{-\mu^{2}}{\lambda}}$ is called the vacuum expectation value of the scalar boson field $\Phi$. For most fields the energy is minimized when the field is set to zero. The construction of the Higgs field is unusual in this respect since at the lowest energy state the field $\Phi$ has a non-zero constant value. The potential $V$ is symmetric with respect to $\Phi$ for both $\mu^{2}<0$ and $\mu^{2}>0$ scenarios. However, for $\mu^{2}<0$ the distribution has two minima at $\pm v$. In weak interactions, calculations can be done by evaluating small perturbations about either of the two minima, $\Phi=+v$ or $\Phi=-v$. When a particular value of the minimum is arbitrarily chosen, the symmetry of the scalar potential $V$ is broken, and is known as spontaneous symmetry breaking.

The complex fields representing the scalar Higgs doublet can be written as $\phi^{+}=$ $\frac{1}{\sqrt{2}}\left(\phi_{1}+\phi_{2}\right)$ and $\phi^{0}=\frac{1}{\sqrt{2}}\left(\phi_{3}+\phi_{4}\right)$, and the anti-particle states are given by $\phi^{-}=\left(\phi^{+}\right)^{*}$ and $\bar{\phi}^{0}=\left(\phi^{0}\right)^{*}$. Three of the four components, $\phi^{+}, \phi^{-}, \phi^{0}$ and $\bar{\phi}^{0}$ couple with the $W^{ \pm}$and the $Z$ bosons, assigning masses to the weak gauge bosons in the process. 
The fourth component manifests itself as the Higgs boson that is yet to be discovered. Recent search results for the Higgs Boson hint that, if it exists, the mass is likely to be in the range $124-126 \mathrm{GeV} / \mathrm{c}^{2}[5,6]$, however, the results are not yet sufficiently significant to claim a discovery.

As mentioned earlier, the phenomenon of spontaneous symmetry breaking assigns masses to the gauge bosons, $W^{ \pm}$and $Z^{0}$, of the electroweak interaction. The masses of the electroweak gauge bosons are related by:

$$
M_{W}^{2}=\rho M_{Z}^{2} / \cos ^{2} \theta_{W}
$$

where $\theta_{W}$ is the weak mixing angle, $M_{W}$ is the mass of the $W^{ \pm}$and $M_{Z}$ denotes the mass of the $Z^{0}$ boson. The parameter $\rho$ is unity to first order but higher order corrections can be calculated using perturbation theory.

Another important consequence of the phenomenon is that the masses of the charged fermions are related to their coupling to the Higgs field. A stronger coupling of a fermion to the Higgs field results in a higher mass of the fermion. In addition, the mass eigenstates of the down, strange and bottom quarks are different from their electroweak eigenstates, and these two states are related via the CKM matrix as discussed in the next section.

\subsubsection{The Cabibbo-Kobayashi-Maskawa (CKM) matrix}

The electroweak eigenstates $d_{L}^{\prime}, s_{L}^{\prime}$ and $b_{L}^{\prime}$ shown in Equation 2.9 are not the same as the mass eigenstates $d, s$ and $b$ described by QCD. The two eigenstates are related by a unitary transformation that can be expressed using the unitary CabibboKobayashi-Maskawa (CKM) matrix:

$$
\left(\begin{array}{l}
d^{\prime} \\
s^{\prime} \\
b^{\prime}
\end{array}\right)=\left(\begin{array}{ccc}
V_{u d} & V_{u s} & V_{u b} \\
V_{c d} & V_{c s} & V_{c b} \\
V_{t d} & V_{t s} & V_{t b}
\end{array}\right)\left(\begin{array}{l}
d \\
s \\
b
\end{array}\right)
$$


For the first two generations of quarks, the unitary transformation can be described using a $2 \times 2$ matrix that can be expressed in terms of a single parameter, the Cabibbo mixing angle $\theta_{C}$ as:

$$
\left(\begin{array}{l}
d^{\prime} \\
s^{\prime}
\end{array}\right)=\left(\begin{array}{cc}
\cos \theta_{C} & \sin \theta_{C} \\
-\sin \theta_{C} & \cos \theta_{C}
\end{array}\right)\left(\begin{array}{l}
d \\
s
\end{array}\right)
$$

The full CKM matrix describing quark mixing in all the three generations can be parametrized using three mixing angles $\theta_{12}, \theta_{23}$ and $\theta_{13}$ and a complex phase $\delta$. Out of the many possible conventions, a standard choice [7] for expressing the parameterization is:

$$
V_{C K M}=\left(\begin{array}{ccc}
c_{12} c_{13} & s_{12} c_{13} & s_{13} e^{-i \delta} \\
-s_{12} c_{23}-c_{12} s_{23} s_{13} e^{i \delta} & c_{12} c_{23}-s_{12} s_{23} s_{13} e^{i \delta} & s_{23} c_{13} \\
s_{12} s_{23}-c_{12} c_{23} s_{13} e^{i \delta} & -c_{12} s_{23}-s_{12} c_{23} s_{13} e^{i \delta} & c_{23} c_{13}
\end{array}\right)
$$

where $s_{i j}=\sin \theta_{i j}$ and $c_{i j}=\cos \theta_{i j}$. The angles $\theta_{i j}$ can be chosen to lie in the first quadrant such that $s_{i j}, c_{i j} \geq 0$. The complex phase $\delta$ gives rise to the chargeparity $(\mathrm{CP})$ violation effects observed in flavor changing process in the Standard Model. Several experimental results have shown that $s_{13} \ll s_{23} \ll s_{12} \ll 1$, and it is convenient to express this hierarchy using the Wolfenstein parameterization [8], in which each element of the matrix is expanded as a power series in $\lambda=\sin \theta_{C}$ :

$$
V_{C K M}=\left(\begin{array}{ccc}
1-\frac{\lambda^{2}}{2} & \lambda & A \lambda^{3}(\rho-i \eta) \\
-\lambda & 1-\frac{\lambda^{2}}{2} & A \lambda^{2} \\
A \lambda^{3}(1-\rho-i \eta) & -A \lambda^{2} & 1
\end{array}\right)+\mathcal{O}\left(\lambda^{4}\right)
$$

where the following definitions are used:

$$
\begin{gathered}
s_{12}=\lambda=\sin \theta_{C}=\frac{\left|V_{u s}\right|}{\sqrt{\left|V_{u d}\right|^{2}+\left|V_{u s}\right|^{2}}} \\
s_{23}=A \lambda^{2}=\lambda\left|\frac{V_{c b}}{V_{u s}}\right| \\
s_{13} e^{i \delta}=A \lambda^{3}(\rho+i \eta)=V_{u b}^{*}
\end{gathered}
$$


The Wolfenstein parameterization is accurate to third order in $\lambda$ and is defined by four independent parameters $\lambda, A, \rho$ and $\eta$ as shown in Equation 2.17. The diagonal terms in the matrix are of order unity whereas the off diagonal terms are smaller by factors of $\lambda$ which implies that flavor changing weak decays between generations are suppressed by factors of $\lambda$. The unitarity of the CKM matrix requires that:

$$
\begin{aligned}
& \sum_{i} V_{i j} V_{i k}^{*}=\delta_{j k} \\
& \sum_{j} V_{i j} V_{k j}^{*}=\delta_{i k}
\end{aligned}
$$

where $\delta_{i i}=1$ and $\delta_{i j}=0$. The six vanishing combinations can be represented as triangles in a complex plane. All the triangles have the same area which represents the magnitude of the CP violating effects in the Standard Model. One of the unitary triangles can be constructed using the following condition:

$$
\begin{gathered}
V_{u d} V_{u b}^{*}+V_{c d} V_{c b}^{*}+V_{t d} V_{t b}^{*}=0 \\
\frac{V_{u d} V_{u b}^{*}}{V_{c d} V_{c b}^{*}}+\frac{V_{t d} V_{t b}^{*}}{V_{c d} V_{c b}^{*}}+1=0
\end{gathered}
$$

The triangle is approximately equilateral, with vertices given by $(0,0),(1,0)$ and $(\bar{\rho}, \bar{\eta})=\left(1-\lambda^{2} / 2\right)(\rho, \eta)$ as shown in Figure 2.1. The angles in the triangle are given by:

$$
\alpha=\arg \left[-\frac{V_{t d} V_{t b}^{*}}{V_{u d} V_{u b}^{*}}\right], \beta=\arg \left[-\frac{V_{c d} V_{c b}^{*}}{V_{t d} V_{t b}^{*}}\right], \quad \gamma=\arg \left[\frac{V_{u d} V_{u b}^{*}}{V_{c d} V_{c b}^{*}}\right]
$$

The CKM matrix elements are fundamental parameters of the electroweak theory and their precise determination is important as it could be sensitive to physics beyond the Standard Model. Therefore, an important goal of flavor physics has been to overconstrain the unitarity triangle shown in Figure 2.1. For example, if there were more than three generations of quarks in nature, the triangle would not be closed. It would in fact be a polygon with the number of sides equal to the number of generations. Since CP violation involves the complex phases of CKM matrix elements, various measurements of $\mathrm{CP}$ violating observables can be used to constrain the angles of the unitary triangle and the $(\bar{\rho}, \bar{\eta})$ parameters. 


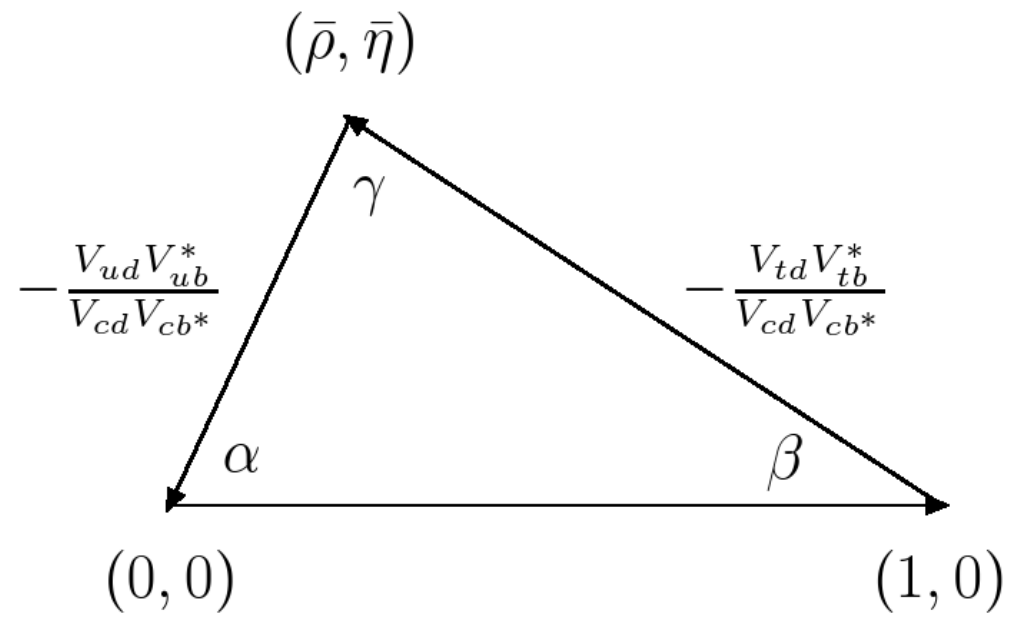

Figure 2.1.: A sketch of the unitary triangle resulting from the relation in Equation 2.20.

The elements of the CKM matrix can be measured by studying weak interactions between hadrons. However, the elements are generally measured by studying semi-leptonic decays of hadrons. Pure hadronic decays have a disadvantage that the momentum distribution of quarks bound inside hadrons needs to be well understood in order to make precise measurements. The momentum distribution is difficult to obtain since perturbative QCD calculations are not applicable at momentum scales of quarks constituting the hadrons. This problem is partly solved by using semi-leptonic decays since the leptonic states are described very well by the electroweak theory.

The amplitude [4,9] for a typical semi-leptonic decay such as $q \rightarrow q^{\prime} l^{-} \bar{\nu}_{l}$ is given by:

$$
\mathcal{M} \sim\left[\bar{u}(l) \gamma_{\mu}\left(1-\gamma_{5}\right) u\left(\bar{\nu}_{l}\right)\right] \frac{1}{M_{W}^{2}-q^{2}}\left[\bar{u}\left(\bar{q}^{\prime}\right) \gamma^{\mu}\left(1-\gamma_{5}\right) V_{q q^{\prime}} u(q)\right]
$$

where $V_{q q^{\prime}}$ is the relevant CKM matrix element, $u(q), \bar{u}(q)$ are the quark creation and annihilation operators, and $u(l), \bar{u}(l)$ are the leptonic creation and annihilation operators. The hadronic vertex in the interaction depends on the momentum transfer $q^{2}$ of the process that is parametrized using a function called the form factor. Table 2.2 
gives an overview of the processes that have been used for the determination $[1,9]$ of the CKM matrix elements.

Table 2.2: An overview of the processes used for the determination of the CKM matrix elements.

\begin{tabular}{|c|l|}
\hline Element & Determination process \\
\hline$V_{u d}$ & Nuclear $\beta$ decays and $\pi^{+} \rightarrow \pi^{0} e^{+} \nu$ \\
$V_{u s}$ & Semi-leptonic kaon and hyperon decays \\
& $\left(K_{L}^{0}, K^{ \pm}, K_{s}^{0} \rightarrow \pi e \nu, \pi \mu \nu\right)$ \\
$V_{u b}$ & Inclusive $B \rightarrow X_{u} l \bar{\nu}$ and $B \rightarrow \pi l \bar{\nu}$ exclusive decays \\
$V_{c d}$ & $D \rightarrow K l \nu, D \rightarrow \pi l \nu$ and $\nu-\bar{\nu}$ interactions \\
$V_{c s}$ & $W^{+} \rightarrow c \bar{s}$ decays, \\
& semi-leptonic $D$ or leptonic $D_{s}$ decays \\
$V_{c b}$ & Exclusive and inclusive semi-leptonic \\
& decays of $B$ mesons to charm \\
$V_{t d}$ & $B^{0}-\bar{B}^{0}$ oscillations and $\left|V_{t d} / V_{t s}\right|$ ratio \\
$V_{t s}$ & $B_{s}^{0}-\bar{B}_{s}^{0}$ oscillations and $\left|V_{t d} / V_{t s}\right|$ ratio \\
$V_{t b}$ & Top decays $(t \rightarrow W q)$ and single-top \\
& quark production cross section measurement \\
\hline
\end{tabular}

The most precise values of the CKM matrix elements are determined by performing a global fit that uses all the available measurements and imposes Standard Model constraints such as unitarity of the three generation CKM matrix. The current best estimates of the CKM matrix elements are:

$$
V_{C K M}=\left(\begin{array}{ccc}
0.97428 \pm 0.00015 & 0.2253 \pm 0.0007 & 0.00347_{-0.00012}^{+0.00016} \\
0.2252 \pm 0.0007 & 0.97345_{-0.00016}^{+0.00015} & 0.0410_{-0.0007}^{+0.0011} \\
0.00862_{-0.00020}^{+0.00026} & 0.0403_{-0.0007}^{+0.0011} & 0.999152_{-0.000045}^{+0.000030}
\end{array}\right)
$$


The CKM matrix elements are important parameters as they influence the rates of the flavor transitions between the three generations of quarks. These flavor changing electroweak interactions play a vital role in the determination of the properties of $\mathrm{B}$ hadrons such as their lifetimes and branching fractions of various decay modes.

\subsection{Summary}

The Standard Model is a gauge theory that provides an excellent description of the properties of elementary particles and three of the four known forces in nature. The gauge theory describes the interactions between quarks at very short distances or high momentum transfers extremely well. However, we are unable to use the theory to make calculations for describing low momentum transfer processes such the quark fragmentation phenomenon, which is the primary focus of the study presented in this dissertation. Various phenomenological models based on QCD have been developed that are used to describe the quark fragmentation phenomenon as discussed in the Chapter 3 . 


\section{QUARK FRAGMENTATION}

QCD is a well established theory that describes the strong interaction but there are some aspects where the theory only provides qualitative predictions. The QCD Lagrangian describes the fundamental properties of quarks and gluons but experimentally we can only directly observe hadrons in a detector. Perturbative QCD calculations can be used to describe the properties of quarks and gluons at small distances or high momentum transfers, but since the strong coupling constant becomes large at large distances, perturbation theory breaks down and fails to describe the interaction between quarks and gluons as they move apart. This leaves a gap in the theoretical description of the process by which partons produced in a high energy interaction are transformed into the final observable hadrons.

A schematic representation of a high energy interaction showing the various phases that lead to the formation of final state hadrons from initial state partons is shown in Figure 3.1. The phenomenological picture of a typical high energy interaction consists of an initial phase involving parton production, which can be described using perturbative QCD. This is followed by a non-perturbative phase in which partons recede from each other and are combined via the strong force to form colorless hadrons. This non-perturbative phenomenon is known as quark fragmentation or hadronization. The colorless hadrons formed in the fragmentation process subsequently decay to lighter mesons via the strong and then the electroweak interactions as illustrated in Figure 3.1.

\subsection{Initial parton shower}

Hadronic collisions, such as $p \bar{p}$ collisions, involve interactions of their constituent quarks and gluons with high momentum transfers. At these energy scales the strong 


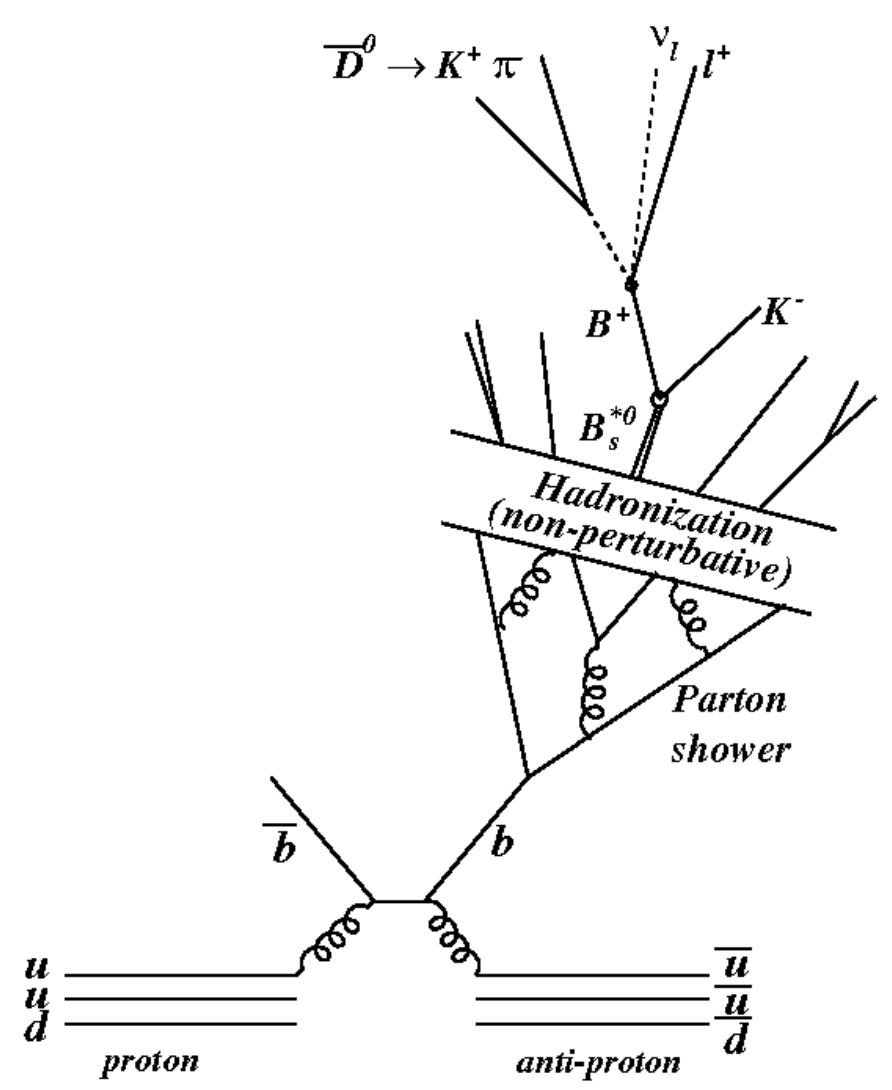

Figure 3.1.: Schematic representation of the production of bottom quarks in a $p \bar{p}$ interaction, which subsequently fragment to form b-hadrons that undergo strong and then weak decays to lighter mesons.

coupling constant, $\alpha_{s}$, has a value of about 0.1 , which is small enough that perturbative QCD can be used to describe the interactions [9]. The Feynman diagrams for some of the lowest order QCD processes that result in the creation of a $b \bar{b}$ or $c \bar{c}$, via annihilation of two lighter quarks or fusion of two gluons are shown in Figure 3.2. The generic expression for calculating the cross-section for the lowest order $2 \rightarrow 2$ processes, such as $q \bar{q} \rightarrow Q \bar{Q}$ can be written as [4]:

$$
\frac{d \hat{\sigma}}{d \hat{t}}(q \bar{q} \rightarrow Q \bar{Q}) \sim|\mathcal{M}|^{2} /\left(16 \pi \hat{s}^{2}\right)
$$


where $\hat{s}$ is the center-of-mass energy squared of the $q \bar{q}$ system and $\hat{t}$ is the square of the difference in the energy of quarks $q$ and $Q$ quarks involved in the $q \bar{q} \rightarrow Q \bar{Q}$ interaction. An important point is that $\hat{s}$ is the center-of-mass energy squared of the colliding quarks, which is not measured directly. It is related to the center-of-mass energy squared $s$ of the colliding hadrons by $\hat{s}=x \cdot s$, where $x$ is the fraction of energy of the colliding hadrons taken up by the colliding quarks. The notation $\mathcal{M}$ represents the amplitude of the $q \bar{q} \rightarrow Q \bar{Q}$ interaction.

The net cross section for all the $2 \rightarrow 2$ interactions between constituent quarks of the colliding hadrons $h_{1} h_{2}$ is obtained by summing over all pairs of initial quarks $q \bar{q}$ and subprocess channels $q \bar{q} \rightarrow Q \bar{Q}$ :

$$
\frac{d \sigma}{d t}\left(h_{1} h_{2} \rightarrow 2 j e t s\right) \sim \sum_{q \bar{q} Q \bar{Q}} \iint d x_{h_{1}} d x_{h_{2}} f_{q / h_{1}}\left(x_{h_{1}}\right) f_{\bar{q} / h_{2}}\left(x_{h_{2}}\right) \frac{d \hat{\sigma}}{d \hat{t}}(q \bar{q} \rightarrow Q \bar{Q})
$$

where $f_{q / h_{1}}\left(x_{h_{1}}\right)$ and $f_{\bar{q} / h_{2}}\left(x_{h_{2}}\right)$ represent the parton densities in the initial colliding hadrons $h_{1}$ and $h_{2}$ respectively.

In addition to the two jet processes, higher order processes such as gluon emission and flavor excitation can also lead to heavy quark production. Figure 3.3 shows the Feynman diagrams for some of the higher order processes that can contribute to the creation of a heavy flavor $Q \bar{Q}$ pair. The overall cross section for these higher order processed can be obtained similar to the 2 -jet cross section by summing over all the possible $a b \rightarrow c d e$ sub-processes:

$$
\frac{d \sigma}{d t}\left(h_{1} h_{2} \rightarrow 3 j e t s\right) \sim \sum_{a b c d e} \iint d x_{h_{1}} d x_{h_{2}} f_{a / h_{1}}\left(x_{h_{1}}\right) f_{b / h_{2}}\left(x_{h_{2}}\right) \frac{d \hat{\sigma}}{d \hat{t}}(a b \rightarrow c d e)
$$

In the leading order 2-jet processes, the quark and antiquark have opposite momenta, but this will not be the case for higher order processes. In addition to $q \bar{q}$, heavy flavor can also be produced via $g g \rightarrow Q \bar{Q}$ interactions as shown in figures 3.2 and 3.3. The quarks in a heavy flavor $Q \bar{Q}$ pair that is produced in a typical high energy interaction, via these 2 -jet and higher order process, subsequently recede and hadronize to form bound states such as mesons and baryons via the quark fragmentation phenomena. 

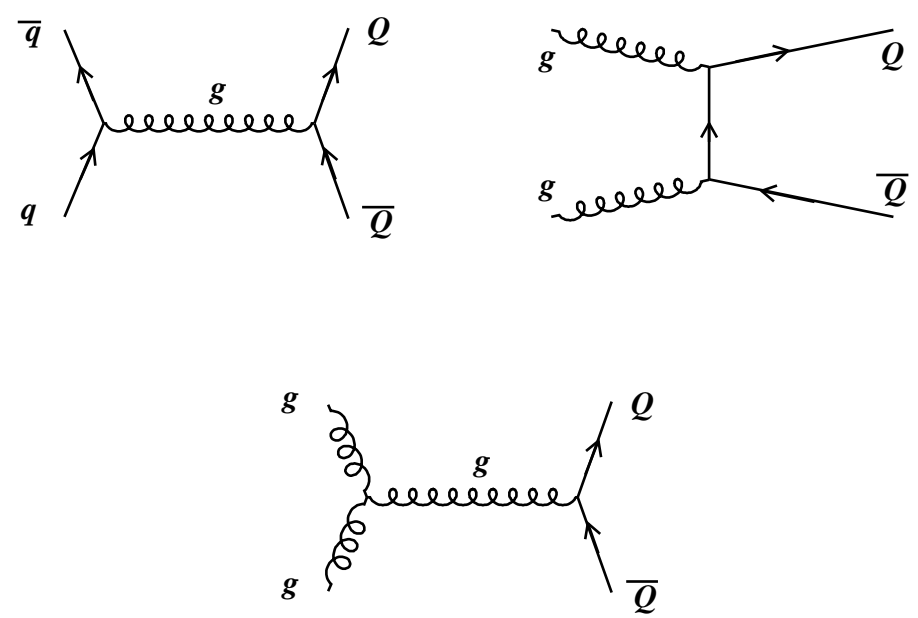

Figure 3.2.: Feynman diagrams for some leading order QCD processes resulting in the creation of heavy quark-antiquark pairs $Q \bar{Q}$.

\subsection{Quark fragmentation}

The fragmentation process is a low momentum, non-perturbative phenomenon with an energy scale of the order of a few $\mathrm{GeV}$. Since perturbative QCD can not be applied at such low energy scales, there is no formal theory that describes the process. Instead, a number of models have been proposed to describe the quark fragmentation phenomena. A brief overview of some of the fragmentation models is presented in the following sections. 

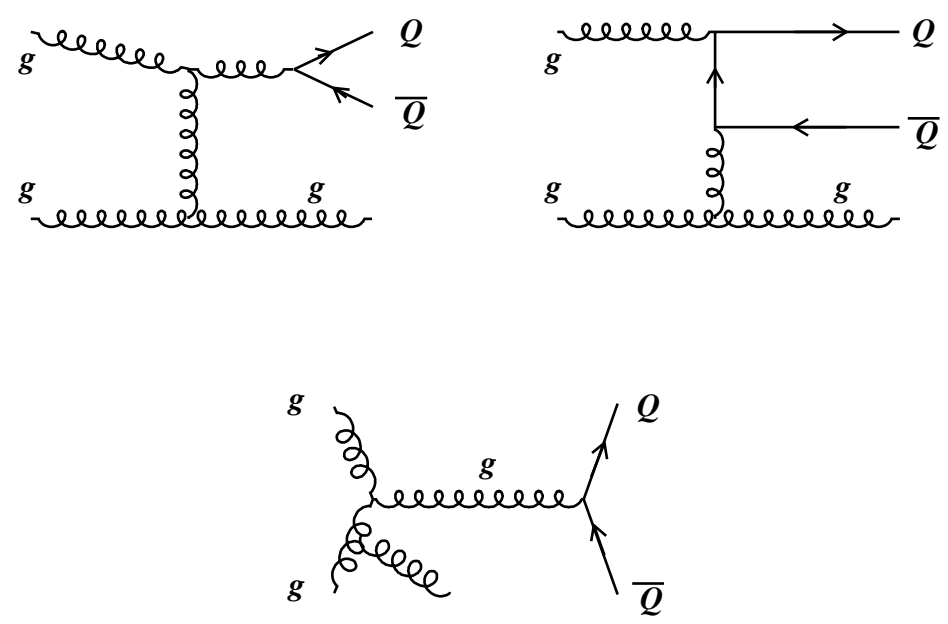

Figure 3.3.: Feynman diagrams for some higher order QCD processes resulting in the creation of heavy quark-antiquark pairs $Q \bar{Q}$.

\subsubsection{The Feynman-Field Model}

The Feynman-Field model is based on the assumption that quark jets produced in the fragmentation process can be analyzed using a recursive principle $[10,11]$. Consider a quark of flavor $q_{0}$ with momentum $P_{0}$ that is moving away from an antiquark. In this process, new quark-antiquark pairs are created from the field energy between them. Subsequently, quark $q_{0}$ then combines with an antiquark $\bar{q}_{1}$ from the new pair $q_{1} \bar{q}_{1}$ to form a meson $q_{0} \bar{q}_{1}$ leaving the remaining quark $q_{1}$ to combine with further anti-quarks. A hierarchy of mesons is formed of which $q_{0} \bar{q}_{1}$ is first in rank, $q_{1} \bar{q}_{2}$ is second in rank, $q_{2} \bar{q}_{3}$ is third in rank, and so on. These are referred to as primary mesons since they may be unstable resonances which eventually decay into more stable secondary mesons. The rank of a meson is assigned in the order of flavor production and 
not in the order of momentum. A rank two primary meson may sometimes obtain a larger momentum than a rank one primary meson. If the rank one meson $q_{0} \bar{q}_{1}$ carries a fraction $z$ of the initial quark $q_{0}$ momentum $P_{0}$, then the remaining cascade starts with a quark of flavor $q_{1}$ with momentum $P_{1}=(1-z) P_{0}$. This recursive process continues until the momentum $P_{n}=\left(1-z_{n}\right) P_{n-1}$ of the $n$th quark falls below a cutoff value and the recursion stops. The model assumes that for very high momenta, all distributions scale so that they depend only on ratios of the hadron momenta to the quark momenta.

The structure of a quark jet can be described by one arbitrary function $f(\eta)$ and three parameters describing the flavor, primary meson spin and the transverse momentum. The function $f(\eta)$ is defined such that $f(\eta) d \eta$ is the probability that the rank one primary meson leaves a fraction $\eta$ of its momentum to the remaining cascade and is normalized:

$$
\int_{0}^{1} f(\eta) d \eta=1
$$

The single particle distribution $F(z)$ is defined so that $F(z) d z$ is the probability of finding a meson, independent of hierarchy, with fractional momentum $z$ within $d z$ in a jet.

Then, $F(z)$ must satisfy the following integral equation

$$
F(z)=f(1-z)+\int_{z}^{1} f(\eta) F(z / \eta) \frac{d \eta}{\eta}
$$

The probability that the meson can be of rank one is then given by $f(1-z) d z$, and if it leaves a momentum fraction $\eta$ with probability $f(\eta) d \eta$, then in the remaining cascade the probability of finding a meson with fractional momentum $z$ in $d z$ is given by $F(z / \eta) d z / \eta$. A nice consequence of this formulation is that

$$
\int_{0}^{1} z F(z) d z=1
$$

The functional form of $f(\eta)$ is selected to be

$$
f(\eta)=(d+1) \eta^{d}
$$


which results in

$$
z F(z)=f(1-z)=(d+1)(1-z)^{d}
$$

The power of $d=2$ gives a good qualitative description of the data for mesons made out of light quarks such as up, down and strange quarks, but the model does not include a description of heavy quark fragmentation or baryon emission.

\subsubsection{The Lund String Model}

The basic principle of the string fragmentation scheme involves the creation of additional quark-antiquark pairs by the color force field $[12,13]$. Let us consider a quark-antiquark pair $\left(q_{1} \bar{q}_{1}\right)$ produced in a high energy interaction. Due to self coupling nature of gluons, the color flux lines between $q_{1}$ and $\bar{q}_{1}$ will be constrained in a thin tube-like region as shown in Figure 3.4, unlike the electromagnetic field lines which spread out over all space. As the $q_{1} \bar{q}_{1}$ pair is pulled apart, the strong coupling constant grows, stretching the color flux lines, and the energy of the color field between the pair increases. Eventually, the energy of the color field between the quark and anti-quark becomes large enough to create a new quark antiquark pair $q_{2} \bar{q}_{2}$ from the available field energy and produces two new strings connecting $q_{1} \bar{q}_{2}$ and $q_{2} \bar{q}_{1}$. If the color field between $q_{1} \bar{q}_{2}$ and $q_{2} \bar{q}_{1}$ has sufficient energy, the flux string can further fragment to create new quark-antiquark pairs. Hence, the original string repeatedly fragments into smaller strings as long as the energy in the string pieces exceeds the invariant mass of a hadron.

Once the field energy is comparable to hadronic masses, the fragmentation process ceases and only colorless hadrons remain. Some of the hadrons that are initially produced may be unstable, such as bottom and charm mesons, and decay further into more stable hadrons (such as pions, kaons), leptons or photons that are finally observed in the detector. The hadrons that are produced in the fragmentation process form a jet of particles that have small transverse momentum relative to the direction of motion of their parent quark that was created in a high energy interaction. In the 


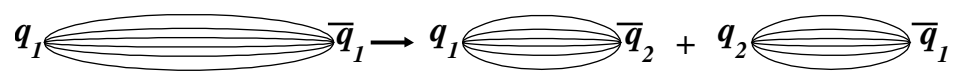

Figure 3.4.: Lines of force of the gluon field between a $q_{1} \bar{q}_{1}$ pair created in a high energy interaction. The gluon flux lines fragment as $q_{1}$ and $\bar{q}_{1}$ move apart, creating quark antiquark pair $q_{2} \bar{q}_{2}$ at the breaking point.

longitudinal direction, the sum of the momenta of the hadrons is roughly equal to the momentum of the parent quark.

At the fragmentation point, the quark-antiquark pairs are created according to a probability that depends on the transverse mass $m_{\perp}^{2}=m^{2}+p_{\perp}^{2}$, and the string constant or energy per unit length, $\kappa \approx 1 \mathrm{GeV} / \mathrm{fm}$. The probability of quark-antiquark pair production is suppressed by a factor:

$$
e^{-\pi m_{\perp}^{2} / \kappa}=e^{-\pi m^{2} / \kappa} e^{-\pi p_{\perp}^{2} / \kappa}
$$

As shown in the equation above, the dependence on the quark mass $m$ suppresses the production of heavier quarks. The transverse momentum $p_{\perp}$ is locally compensated between the quark and the antiquark. In the string fragmentation model, the light cone energy-momentum fraction is defined as:

$$
z=\frac{\left(E+p_{\|}\right)_{h}}{(E+p)_{q}}
$$

where $h$ represents a hadron that is formed by the combination of an energetic quark $q$ with other lighter quarks or anti-quarks pulled out of the vacuum, $p_{\|}$denotes the 
momentum of the hadron $h$ along the direction of the quark $q$. The distribution of $z$ is given by the Lund symmetric fragmentation function:

$$
f_{\alpha \beta}(z)=N_{\alpha \beta} \frac{1}{z} z^{a_{\alpha}}\left(\frac{1-z}{z}\right)^{a_{\beta}} e^{\left(-b m_{\perp}^{2} / z\right)}
$$

where $f_{\alpha \beta}(z)$ gives the probability that a quark with flavor $\alpha$ combines with an antiquark with flavor $\beta$ to form a meson with transverse mass $m_{\perp}$ and energy-momentum fraction $z$. The symbols $a_{\alpha}$ and $a_{\beta}$ are free parameters, one for each flavor. The parameter $b$ is universal and $N_{\alpha \beta}$ is a normalization constant. However, in practice it is customary to equate all $a_{\alpha}$ and $a_{\beta}$ parameters and obtain the following simplified expression:

$$
f(z) \sim \frac{1}{z}(1-z)^{a} e^{\left(-b m_{\perp}^{2} / z\right)}
$$

where $a$ and $b$ are free parameters that can be tuned so that the model predictions are in accordance with the experimental results. The mass dependence in $f(z)$ implies that the fragmentation function decreases faster for heavier hadrons. As the mass increases, the mean $z$ for heavy hadrons asymptotically tends towards:

$$
z \approx 1-\frac{1+a}{b m_{\perp}^{2}}
$$

Although the predictions for charms mesons agrees fairly with the experimental results, the model seems to predict a $z$ distribution for bottom mesons that decreases faster than that observed in data.

\subsubsection{Peterson Fragmentation Model}

Consider a heavy quark-antiquark pair $Q \bar{Q}$ produced in a parton shower following some high energy collision. The initial fragmenting parton is a heavy quark $Q$ (or $\bar{Q})$ and only a small fraction of its energy will be needed to create a number of light quark-antiquark pairs with comparable velocity. Subsequently, the heavy quark can combine with one or more of these light quarks to produce a heavy flavored hadron $H_{Q}$ that will carry a large fraction of the original energy of the quark $Q$ i.e. 
$z=E_{H} / E_{Q} \sim 1$. This implies that the fragmentation of heavy quarks into heavy hadrons will have hard distributions that will be concentrated at large values of $z$. This behavior differs from the fragmentation of light quarks into light hadrons which peaks at smaller values of $z$.

The Peterson fragmentation model is commonly used to describe the explicit features of the heavy quark fragmentation process [14]. Figure 3.5 shows the transition of a heavy quark $Q$ with momentum $P$ to a heavy hadron $H(Q \bar{q})$ plus a spectator light quark $q$ via the creation of a $q \bar{q}$ pair from the energy in the color force field. The

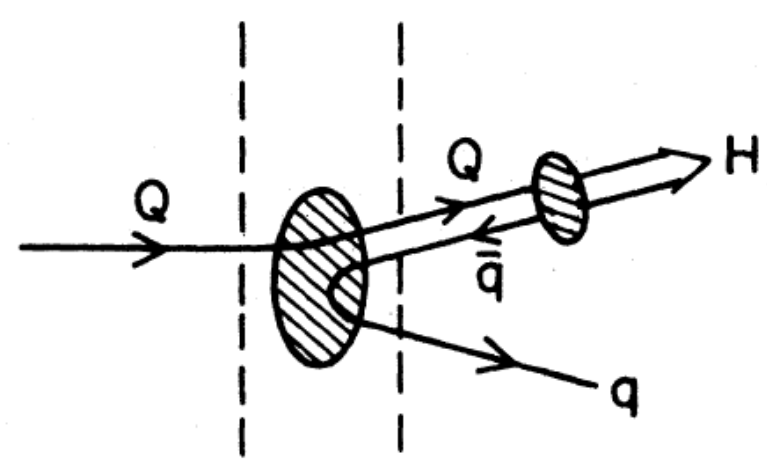

Figure 3.5.: The fragmentation of a heavy quark $Q$ into a meson $H(Q \bar{q})[14]$.

momentum of the hadron $H$ is given by $z P$ where $z$ is the momentum transfer, thus $(1-z) P$ being the momentum of the spectator quark $q$. The model assumes that the probability of the hadronization process $Q \rightarrow H(Q \bar{q})$ is inversely proportional to the energy transfer $\Delta E=E_{H}+E_{q}-E_{Q}$ in the transition

$$
\mathcal{P}(Q \rightarrow H+q) \propto \frac{1}{\Delta E}
$$

Expanding the energy terms in the expression for $\Delta E$ and assuming $m_{H} \approx m_{Q}$ we obtain,

$$
\Delta E=\sqrt{m_{Q}^{2}+z^{2} P^{2}}+\sqrt{m_{q}^{2}+(1-z)^{2} P^{2}}-\sqrt{m_{Q}^{2}+P^{2}}
$$


Assuming that the heavy quark $Q$ fragments at high momentum i.e. $m_{Q} / P \ll 1$,

$$
\begin{gathered}
\Delta E \simeq \frac{m_{Q}^{2}}{2 P}\left(\frac{m_{q}^{2} / m_{Q}^{2}}{(1-z)}+\frac{1}{z}-1\right) \\
\Delta E \propto 1-\frac{1}{z}-\frac{\epsilon_{Q}}{(1-z)}
\end{gathered}
$$

where $\epsilon_{Q}=m_{q}^{2} / m_{Q}^{2}$. Including a factor $1 / z$ for longitudinal phase space, the fragmentation function for heavy quarks is give by

$$
D_{Q}^{H}(z)=\frac{N}{z\left[1-(1 / z)-\epsilon_{Q} /(1-z)\right]^{2}}
$$

where $N$ is the normalization constant. The parameter $\epsilon_{Q}$ is the ratio of the effective light and heavy quark masses. The value of $m_{q}$ is of the order of the non-perturbative strong interaction scale $\sim(0.5-1) m_{p}$ which gives $\epsilon_{Q} \sim(0.1-0.5) / m_{Q}^{2}$. As $\epsilon_{Q}$ decreases, the peak of the fragmentation function moves closer to $z=1$. An example of the Peterson fragmentation function is shown in Figure 3.6. The momentum transfer, $z$, spectrum is harder for a bottom quark as compared to the charm quark.

\subsubsection{Cluster Fragmentation Model}

In the cluster fragmentation model, the gluons that are produced in the final stages of the parton shower are split into $q \bar{q}$ pairs. The $q \bar{q}$ pairs recombine with other nearby quarks and anti-quarks that are produced by the parton shower to form loosely bound color singlet states. These bound states are referred to as clusters and have mass of the order of $1 \mathrm{GeV} / \mathrm{c}^{2}$, which is the non-perturbative energy scale attained at the end of the parton shower. The phenomenon of formation of clusters is referred to as pre-confinement [15]. Figure 3.7 naively illustrates how the large initial energy $\left(\sqrt{Q^{2}}\right)$ decreases through successive and almost collinear creation of new quark-antiquark pairs. The creation of new pairs continue till the threshold of $\sqrt{Q_{0}^{2}} \sim 1 \mathrm{GeV} / \mathrm{c}^{2}$ is reached.

The clusters usually decay into one or two lighter stable hadrons, whereas heavier clusters can decay into two clusters. The decay of a cluster into two hadrons is 


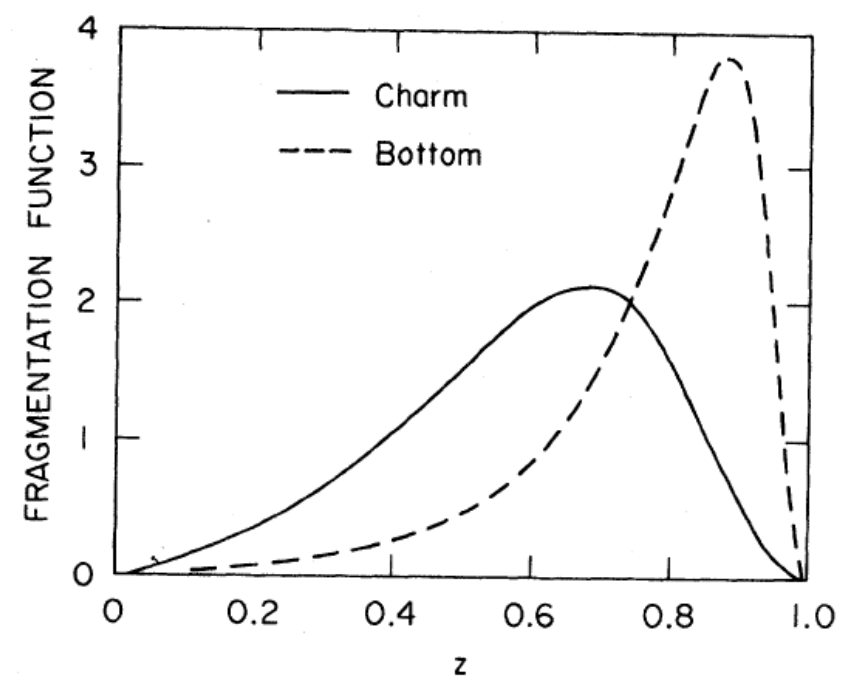

Figure 3.6.: Fragmentation functions $D_{c}(z)$ and $D_{b}(z)$ for charm and bottom quarks. The functional form is shown in Equation 3.17. The distributions are made using $\epsilon_{c}=0.15$ and $\epsilon_{b}=0.15\left(m_{c} / m_{b}\right)^{2}=0.016$ [14].

assumed to be isotropic in the rest frame of the cluster. The hadron type or decay channel of the cluster is determined by the available phase space. The cluster fragmentation model has an advantage of having few parameters and a simple mechanism for generation of the momentum spectra.

\subsection{Summary}

Quark fragmentation is a low momentum process that is not calculable using perturbative QCD, but various phenomenological models have been developed based on QCD in order to describe the non-perturbative process. Since some experimental techniques rely on aspects of fragmentation that we anticipate, it is useful and interesting to study to what extent the models describe observations. The research described in this dissertation explores an aspect of fragmentation that has not previously been studied, which is relevant for analyses of B-mesons at hadron colliders. 


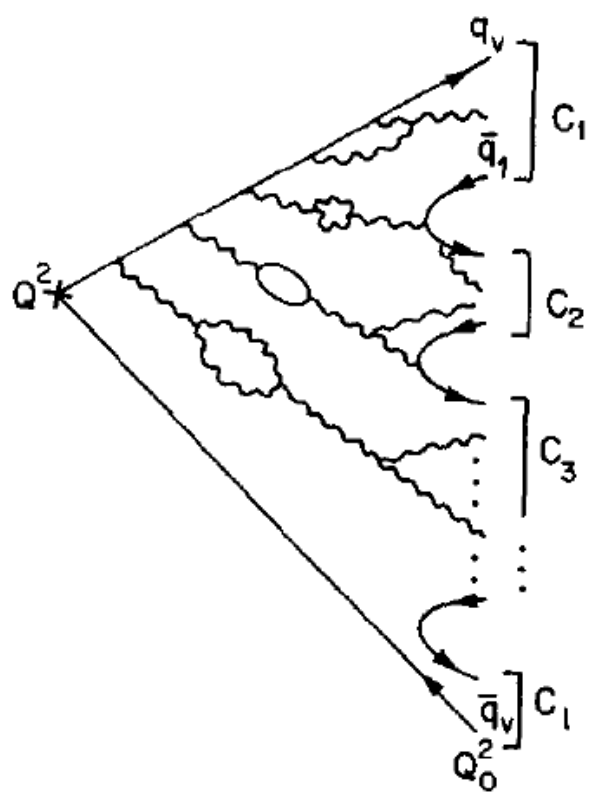

Figure 3.7.: Example diagram showing evolution of jets using cluster fragmentation model, in a typical high energy interaction. Final partons $q_{v}, \overline{q_{1}} \ldots \overline{q_{v}}$ have energy of the order of few $\mathrm{GeV}$ and combine to form color singlet clusters $C_{i}$ [15]. 


\section{EXPERIMENTAL APPARATUS}

The experimental apparatus used in our analysis is the upgraded Collider Detector at Fermilab, also known as CDF II, which observes the results of high energy $p \bar{p}$ collisions, allowing us to study various fundamental physical phenomena. This chapter provides an overview of the accelerator complex which produces proton-antiproton collisions in the CDF II detector and also provides a description of the various components of the detector.

\subsection{The Accelerator Complex}

The purpose of the accelerator complex is to produce protons and anti-protons, accelerate them to $980 \mathrm{GeV}$ and deliver the high energy beam to various experiments [16]. The particle beams are delivered to the CDF experiment where $p \bar{p}$ collisions can occur at a center of mass energy of $\sqrt{s}=1.96 \mathrm{TeV}$. The configuration of the accelerators used at Fermilab is shown in Figure 4.1. The accelerator complex consists of several components since particles can not be created and accelerated from rest to $980 \mathrm{GeV}$ in the same device. This section presents a brief description of the various components of the accelerator complex.

\subsubsection{Pre-Accelerator}

The Cockcroft-Walton pre-accelerator is the first accelerator in the complex. The pre-accelerator consists of a source housed in an electrically charged dome that produces negatively charged hydrogen ions $\left(H^{-}\right)$by ionizing hydrogen gas. The dome is charged to a potential of $-750 \mathrm{keV}$ and accelerates the $H^{-}$ions to an energy of $750 \mathrm{keV}$ through a column from the charged dome towards a grounded wall. The 


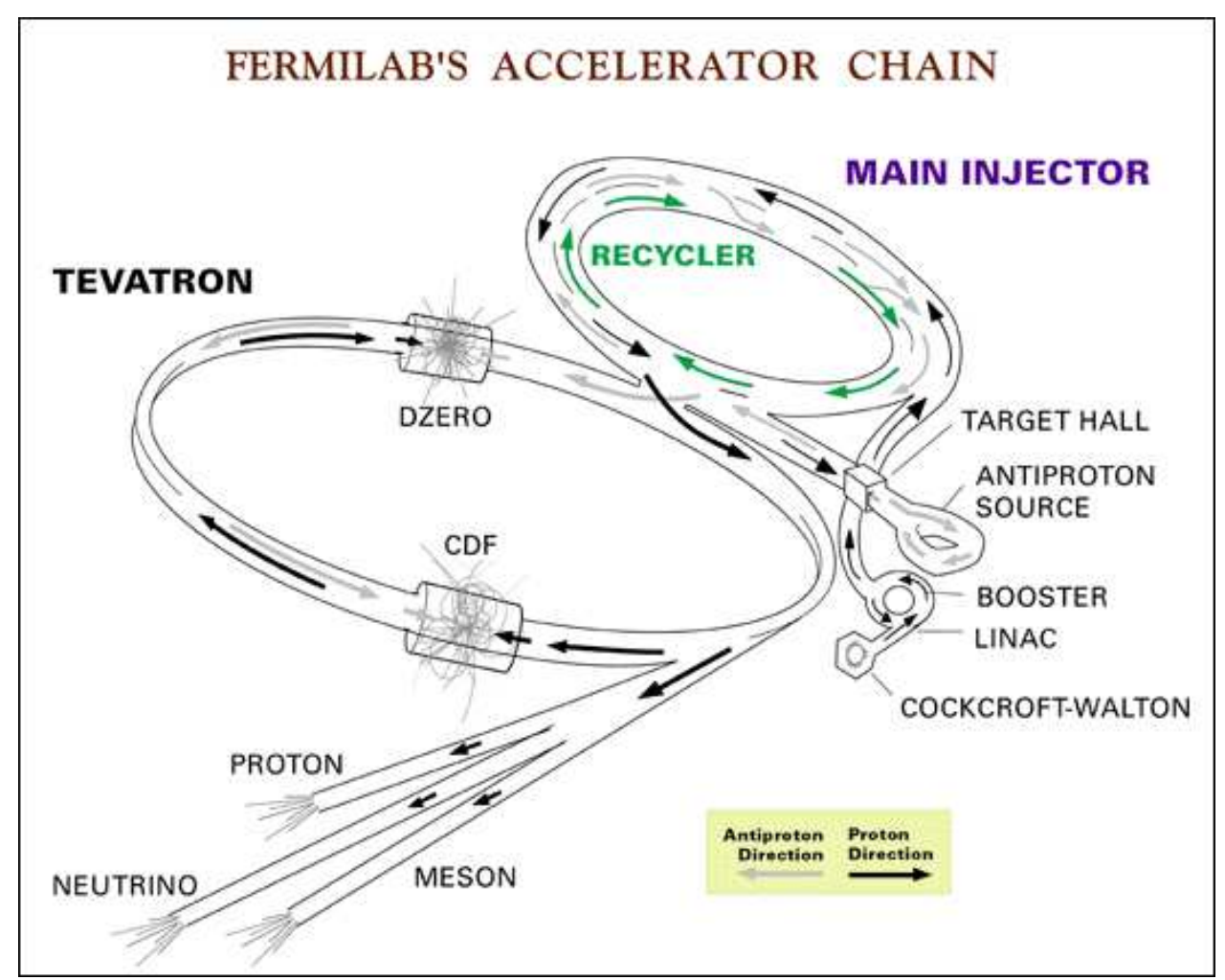

Figure 4.1.: Overview of the accelerator complex at the Fermi National Accelerator Laboratory (Fermilab) [16].

basic design of the accelerator consists of an assembly of voltage doubler circuits and the ions are accelerated by the potential differences between the stages of the assembly [17]. After the beam of $H^{-}$ions exits the accelerating column, it is delivered to a linear accelerator.

\subsubsection{Linear Accelerator}

The linear accelerator is approximately 500 feet long and accelerates $H^{-}$ions with an initial energy of $750 \mathrm{keV}$ to a final energy of $400 \mathrm{MeV}$. The Linac consists of two main accelerating components, the low energy drift tube Linac (DTL) consisting of the first five RF stations and the high energy side-coupled cavity Linac (SCL) which 
consists of the last seven RF stations. The DTL accelerates $H^{-}$ions from $750 \mathrm{keV}$ to $116 \mathrm{MeV}$ using oscillating electric fields applied to drift tubes. The side-coupled cavity Linac (SCL) takes the $116 \mathrm{MeV}$ beam of $\mathrm{H}^{-}$ions from the drift tube cavities and accelerates the beam to $400 \mathrm{MeV}$. The SCL consists of several side-coupled cavity modules. The modules are not one cavity like the drift tubes, but rather several separate cavities powered by the same RF source by coupling.

Bunching is a natural feature of the Linac since the oscillating electric field in a drift tube pushes back the slow $H^{-}$ions, which do not enter the drift tube before the electric field points in the decelerating direction. On the other hand, the ions that traverse the gap between the drift tubes and enter the next drift tube while the electric field is pointing in the positive direction are accelerated. Next, the final $400 \mathrm{MeV}$ beam of $\mathrm{H}^{-}$ions is transferred to the Booster.

\subsubsection{Booster}

The Booster is the first circular accelerator, or synchrotron, in the chain of accelerators and has a diameter of 150 meters. It is made up of 96 magnets in a series of 24 repeating periods which bend the beam and also focus the beam either horizontally or vertically. The Booster has $18 \mathrm{RF}$ cavities, located around the Booster ring, interspersed with the magnets. Each RF cavity contains a drift tube with accelerating gaps at both ends.

The Booster accepts the $400 \mathrm{MeV} \mathrm{H}^{-}$ions from the Linac. At injection, the two electrons are stripped off from the $H^{-}$ions by sending them through a carbon foil. The remaining $H^{+}$ions (i.e. protons) are accelerated to an energy of $8 \mathrm{GeV}$.

The frequency of the bunches from the Linac does not match the frequency of the RF cavities in the Booster. Since the bunches arriving from the Linac are out of phase with the Booster RF cavities, the bunch structure from the Linac disappears resulting in a constant line of charge. After all of the beam has been injected into the Booster, the Booster RF cavities eventually come into phase with each other and 
a new bunch structure is formed. Once the beam of proton bunches is accelerated to $8 \mathrm{GeV}$ it is delivered to the Main Injector.

\subsubsection{Main Injector}

The Main Injector is the next stage of the accelerator complex and is used as both a pre-accelerator for injecting protons into the Tevatron and for delivering the proton beam to the anti-proton production target. It is a synchrotron with eighteen accelerating RF cavities and has a circumference of almost two miles.

The Main Injector has numerous dipole and quadrupole magnets and can accelerate $8 \mathrm{GeV}$ protons from the Booster to either $120 \mathrm{GeV}$ or $150 \mathrm{GeV}$, depending on their destination. The $120 \mathrm{GeV}$ proton beam is delivered to the anti-proton source for $\bar{p}$ production, the fixed target and neutrino experiments at Fermilab. The $150 \mathrm{GeV}$ proton beam is injected into the Tevatron. The Main Injector also accepts $8 \mathrm{GeV}$ antiprotons $(\bar{p})$ from the anti-proton source, accelerates them to $150 \mathrm{GeV}$ and delivers the accelerated anti-proton beam to the Tevatron.

\subsubsection{Anti-Proton Production}

The Main Injector provides $120 \mathrm{GeV}$ protons to the anti-proton source for $\bar{p}$ production. The anti-proton source is comprised of several components which collect anti-protons from the target, cool them and store them prior to injection into the Tevatron. Anti-protons are produced by colliding a beam of $120 \mathrm{GeV}$ protons with a nickel alloy target. Various nuclear interactions take place when the high energy protons strike the target resulting in the production of numerous secondary particles. The particles produced in the interactions can be selected based on their momentum and charge using a system of magnets. A beam of $8 \mathrm{GeV}$ anti-protons is collected from the spray of secondary particles and is transferred to the Debuncher. The antiproton production efficiency is about $10^{-6}$ anti-protons per incident proton on the nickel target. 
The Debuncher is a synchrotron with a mean radius of 90 meters. The beam of anti-protons that is transfered to the Debuncher has a large momentum spread. The primary purpose of the Debuncher is to efficiently capture the $8 \mathrm{GeV}$ antiproton bunches from the target station and reduce their momentum spread. Once the energy of the anti-protons is nearly uniform, the Debuncher delivers them to the Accumulator. The Accumulator is a synchrotron with a mean radius of 75 meters and is housed in the same tunnel as the Debuncher. The purpose of the Accumulator, as the name implies, is to accumulate anti-protons at an energy of $8 \mathrm{GeV}$ until they are needed for injection into the Tevatron via the Main Injector.

Anti-proton production was limited by the fact that the emittance of the antiprotons got bigger as more of them were stored in the accumulator. To solve this issue, another larger storage ring known as the Recycler was used for storing and cooling $8 \mathrm{GeV}$ anti-protons [18]. The Recycler is a synchrotron located along the ceiling of the Main Injector tunnel and is built using permanent magnets. It accepts anti-protons from the Accumulator via the Main Injector and cools them using both stochastic and electron cooling, in order to reduce the energy spread of the anti-proton beam. Electron cooling is a process in which a beam of electrons is merged with the anti-proton beam and the random energy of the anti-protons is transfered to the electrons via the Coulomb interaction, reducing the energy spread of the anti-protons. Stochastic cooling uses the electrical signals produced by individual anti-protons in the beam to drive an electro-magnet device, known as an electric kicker. This device kicks individual anti-protons in the beam to reduce the transverse momentum spread. These individual kicks are applied continuously and over an extended period of time, which eventually reduces the overall momentum spread of the anti-proton beam. Hence, the two cooling methods used in the Recycler together reduce the longitudinal and transverse spread of the anti-proton beam. When needed, the $8 \mathrm{GeV}$ anti-protons in the Recycler are sent to the Main Injector for injection into the Tevatron. 


\subsubsection{Tevatron}

The Tevatron is the final and largest component of the accelerator complex, with a circumference of approximately 4 miles. It is a synchrotron with eight accelerating cavities and has cryogenically cooled superconducting magnets for bending and focusing of the particle beams. The purpose of the Tevatron is to accept both protons and anti-protons from the Main Injector, accelerate them from $150 \mathrm{GeV}$ to $980 \mathrm{GeV}$ and collide the two counter-circulating beams.

The protons and anti-protons are circulated in 36 bunches which collide every $396 \mathrm{~ns}$ at two intersection points on the Tevatron ring, known as $B \oslash$ and $D \oslash$. The CDF II detector is located at the $B \oslash$ intersection point and another detector is located at $D \oslash$ that is its namesake. During the bunch crossing one or more $p \bar{p}$ collisions may occur creating secondary particles that are recorded by the two detectors.

\subsection{The Collider Detector at Fermilab}

The upgraded Collider Detector at Fermilab (CDF II) [19] is a general purpose solenoidal detector. An isometric overview of the CDF II detector is presented in Figure 4.2, which shows several main components.

The tracking systems, namely the silicon detectors and the Central Outer Tracker, are contained in a superconducting solenoid. The superconducting solenoid is $1.5 \mathrm{~m}$ in radius, $4.8 \mathrm{~m}$ in length and made of an aluminum-stabilized $\mathrm{NbTi}$ conductor. It is operated with a current of $4650 \mathrm{~A}$ to generate a uniform magnetic field of about 1.4 $\mathrm{T}$ inside the tracking volume. The solenoid is contained within a cryostat where it is cooled by liquid helium. Just outside the tracking system and inside the cryostat is the Time-of-Flight (TOF) detector. The TOF sub-system is followed by the electromagnetic and hadronic calorimeters. The muon chambers are located beyond the calorimeter systems. The various detector sub-systems are briefly described in this section. Another important aspect of the CDF II detector is the trigger system which is discussed in Section 4.2.7. 


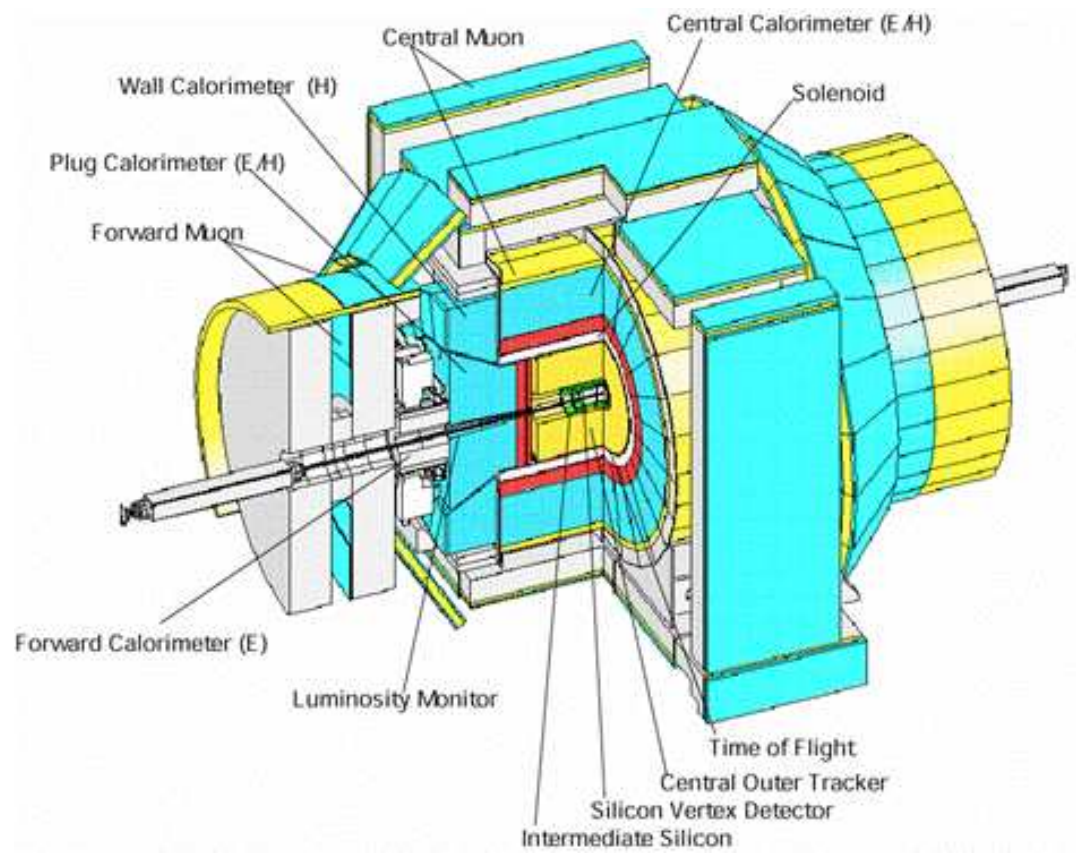

Figure 4.2.: An isometric view of the Collider Detector at Fermilab [19].

Particles created in $p \bar{p}$ collisions pass through the various components of the CDF II detector and can be detected by one or more of the sub-detectors depending on the type of the particles. Figure 4.3 shows a schematic view of the passage and detection of different particles species in some of the sub-detector systems of CDF II.

\subsubsection{Conventions and definitions used at CDF}

The proton and anti-proton beams travel along the horizontal axis and the direction of the proton beam is defined as the positive $z$ direction. The detector is approximately symmetric around the beam axis and the vertical plane intersecting at the collision point. In Cartesian coordinates, the $x$ axis is defined such that it points radially outwards from the center of the Tevatron, while the $y$ axis points upwards. The $x-y$ plane perpendicular to the beam line is referred to as the transverse 


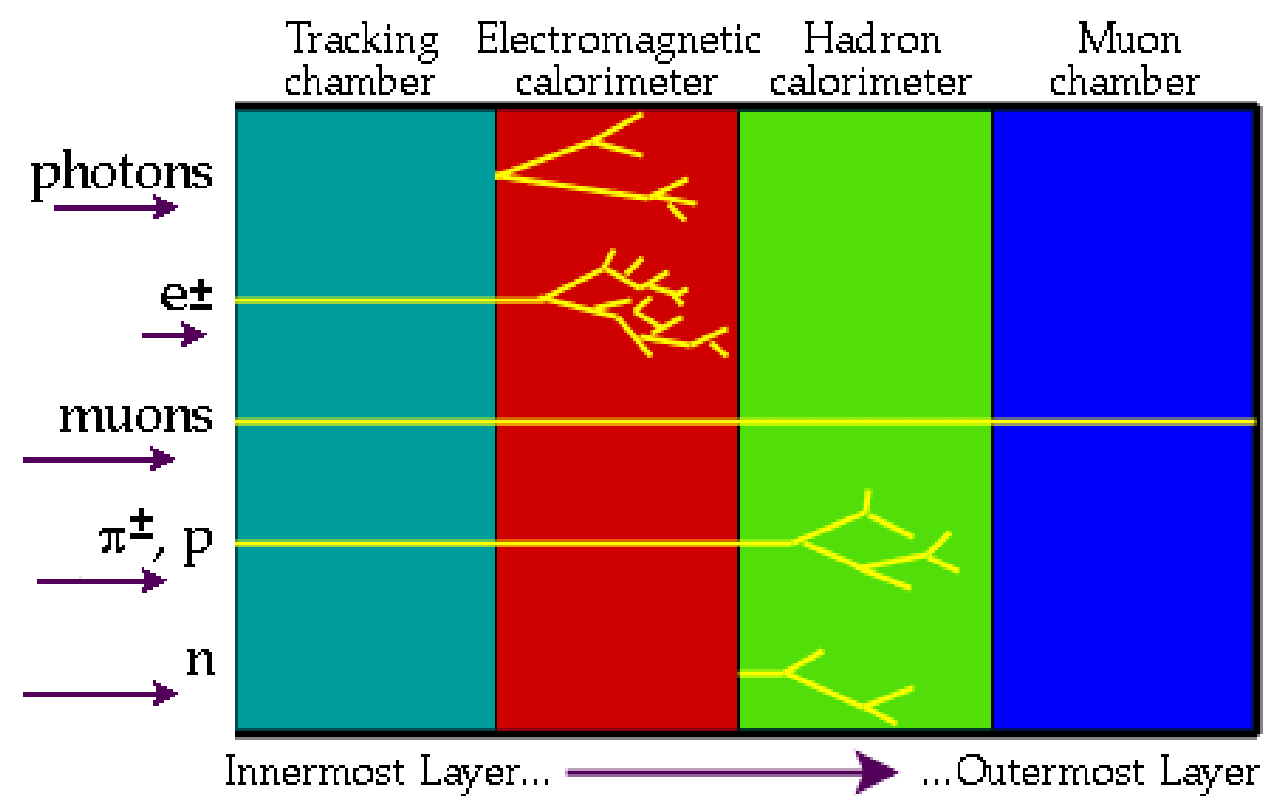

Figure 4.3.: A schematic view showing the passage and detection of different particles in the various sub-systems of CDF II [19].

plane and the projection of a particle's momentum vector onto this plane is called the transverse momentum, $p_{T}$. Because of the cylindrical symmetry, it is sometimes more convenient to use a polar $(r, \phi, \theta)$ or, a cylindrical $(r, \phi, z)$ coordinate system, instead of the Cartesian coordinate system. The quantity $r$ is defined as the radial distance from the center of the detector. The azimuthal angle $\phi$ is defined with respect to the positive $x$ axis and the polar angle $\theta$ is defined with respect to the positive $z$ axis.

In $p \bar{p}$ collisions, the colliding partons carry only a fraction of the kinetic energy of the proton or the anti-proton. Hence, all of the center-of-mass energy of the interacting beams is not absorbed in the collision. Therefore, the center-of-mass system of a parton collision is boosted along the $z$-axis by an unknown amount. However, the quantities defined in the transverse plane are Lorentz invariant in the collisions.

The position of a $p \bar{p}$ collision, from which many tracks originate, is referred to as the primary vertex. The displacement of other vertices with respect to the primary 
vertex is often described by $L_{x y}$, the transverse decay length in the laboratory frame, defined as:

$$
L_{x y}=\vec{r} \cdot \hat{p}_{T}
$$

where $\vec{r}$ is the displacement of the decay vertex in the transverse plane and $\hat{p}_{T}$ is the unit vector in the direction of $\overrightarrow{p_{T}}$. Another variable that is frequently used while referring to the detector geometry is the pseudorapidity, $\eta$, which is defined as:

$$
\eta=-\log \left[\tan \left(\frac{\theta}{2}\right)\right]
$$

\subsubsection{Tracking at CDF}

Tracking is the process of reconstructing the trajectories of charged particles, produced in $p \bar{p}$ collisions, which deposit energy in the detector. This process is central to our analysis as it is used in the reconstruction of charm mesons and associated charged particles. A cross sectional view of the tracking systems at CDF II is shown in Figure 4.4. The innermost tracking system is the silicon vertex detector, which consists of three sub-detectors, described in Section 4.2.3. The second tracking system used at CDF is the Central Outer Tracker, which is described in Section 4.2.4.

A charged particle traveling through the detector looses energy as it ionizes the detector material. The tracking systems detect this ionization and various pattern recognition algorithms can use these signals to map out the trajectory of the particle. Five parameters are used to describe the trajectory of a charged particle traveling through the magnetic field: the curvature of the track $(C)$, the distance of closest approach of the track to the origin or primary vertex of the event $\left(d_{0}\right)$, the azimuthal angle of the track at the point of closest approach $\left(\phi_{0}\right)$, the $z$ position of the track $\left(z_{0}\right)$ at the point of closest approach and the cotangent of the polar angle $(\cot \theta)$. The

values of these five parameters for each track are obtained by performing a fit to the measured pattern of signals in the tracking detectors. 


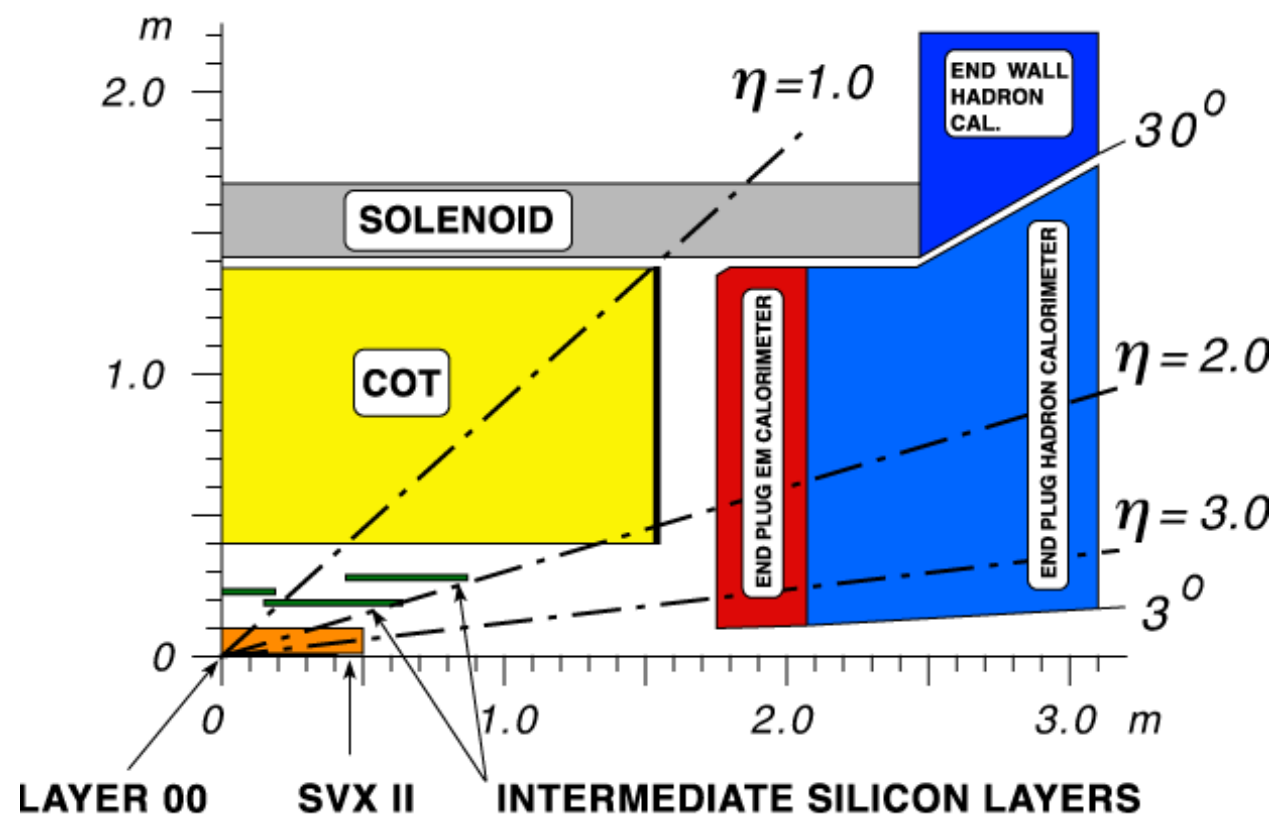

Figure 4.4.: An overview of the tracking layout at CDF II showing the different sub-detector systems [19].

\subsubsection{Silicon Detectors}

The innermost component of the silicon vertex strip detector at CDF is called Layer 00 around which is located the silicon vertex detector (SVX-II) and the Intermediate Silicon Layers (ISL). Figure 4.5 shows a cross-sectional view of the silicon detector system in the $r-\phi$ plane. The $\eta$ coverage of the silicon detector system is shown in Figure 4.6.

The silicon strip detectors at CDF provide a precise determination of the trajectory of a charged particle close to the beam line. Sensors used in the silicon tracking detector are made of silicon substrate doped to form a reverse biased p-n junction. When a charged particle passes through the semi-conductor material of the silicon detector, electron-hole pairs are produced. The electrons drift towards the anode and the holes drift towards the cathode where they are collected. The amount of charge is proportional to the path length traversed by the charged particle in the detector 
material. In these micro-strip detectors, the typical distance between two strips is about $60 \mu \mathrm{m}$ and each layer has a nominal thickness of $300 \mu \mathrm{m}$. The charge deposited by an ionizing particle is referred to as a hit. A particle passing through the silicon detector leaves a hit in one or more adjacent strips creating a hit cluster.

The radial extent of the silicon system is $1.5 \leq r \leq 28 \mathrm{~cm}$ and it extends $\sim$ $90 \mathrm{~cm}$ along the $\mathrm{z}$ axis. The innermost component, Layer 00, consists of one layer of single sided silicon micro-strip sensors oriented parallel to the beam axis providing $r$ - $\phi$ position measurements only. The SVX-II and ISL are made up of double sided micro-strip sensors. The double sided design provides the important benefit of two dimensional information on the location of hit clusters created by an ionizing particle passing through the detector.

The SVX-II consists of five double sided layers of silicon strips arranged in three $29 \mathrm{~cm}$ long barrels. The second and fourth layers of the SVX-II detector have a small stereo angle $\left(1.2^{\circ}\right)$ and the remaining three layers have a $90^{\circ}$ stereo angle relative to the $z$ direction. The layers are positioned at increasing radii and each layer is composed of four silicon sensors, which are aligned length-wise in a ladder structure with readout electronics mounted onto the ends of the ladders. All the SVX-II barrels are divided into 12 azimuthal wedges, each wedge covering $30^{\circ}$ in $\phi$ with a small overlap at the edges. The ISL sub-detector system consists of two layers of double sided silicon sensors arranged outside the SVX-II detector and provides small angle $\left(1.2^{\circ}\right)$ stereo information as well.

\subsubsection{Central Outer Tracker}

The Central Outer Tracker (COT) is a cylindrical drift chamber located between the silicon detector and the Time-of-Flight detector. It is the main tracking device at CDF II and provides accurate tracking information in the $r$ - $\phi$ plane for the mea-

surement of the transverse momentum, $p_{T}$, of a particle. The active volume of the COT begins at a radius of $43 \mathrm{~cm}$ from the beam line and extends out to a radius of 


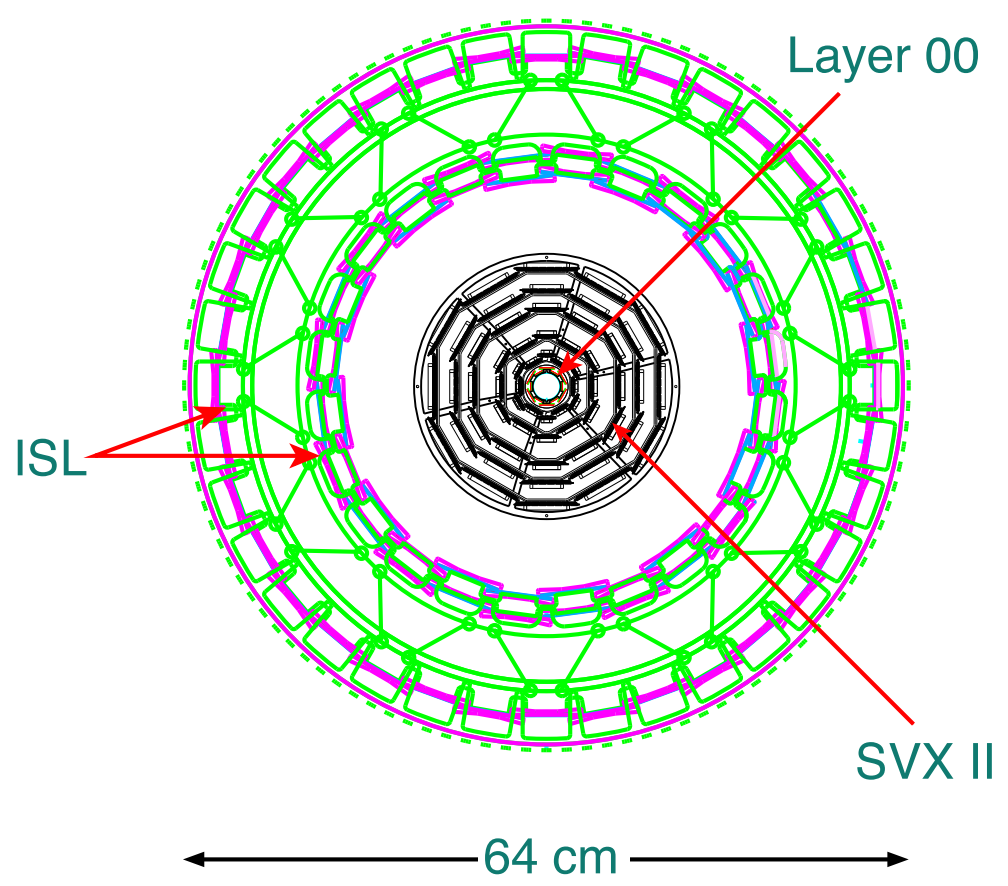

Figure 4.5.: A cross-sectional view of the silicon detector at CDF II showing the three sub-detector systems in the $r-\phi$ plane [19].

$133 \mathrm{~cm}[19,20]$. The chamber is $310 \mathrm{~cm}$ long along the beam line. The COT contains 96 sense wires which are radially grouped into 8 superlayers. Each superlayer is divided into supercells in $\phi$, each of which has 12 sense wires as shown in Figure 4.7. The COT contains 30, 240 sense wires in total that span the entire length of the detector in $z$. Half of the wires are aligned in the $z$ direction (called axial wires) and half run at a small stereo angle $\left(2^{\circ}\right)$ with respect to the $z$ direction (called stereo wires). Particles produced in $p \bar{p}$ collisions having $|\eta|<1$ pass through all the 8 superlayers of the COT, while those with $|\eta|<1.3$ pass through at-least 4 superlayers. Figure 4.8 shows the layout of supercells in the second superlayer of the COT. The supercells consist of sense and potential wires (for electric field shaping) and a field (cathode) sheet on either side. The sense and potential wires are $40 \mu \mathrm{m}$ in diameter and are made of gold plated tungsten. The field sheet is $6.35 \mu \mathrm{m}$ thick Mylar with 


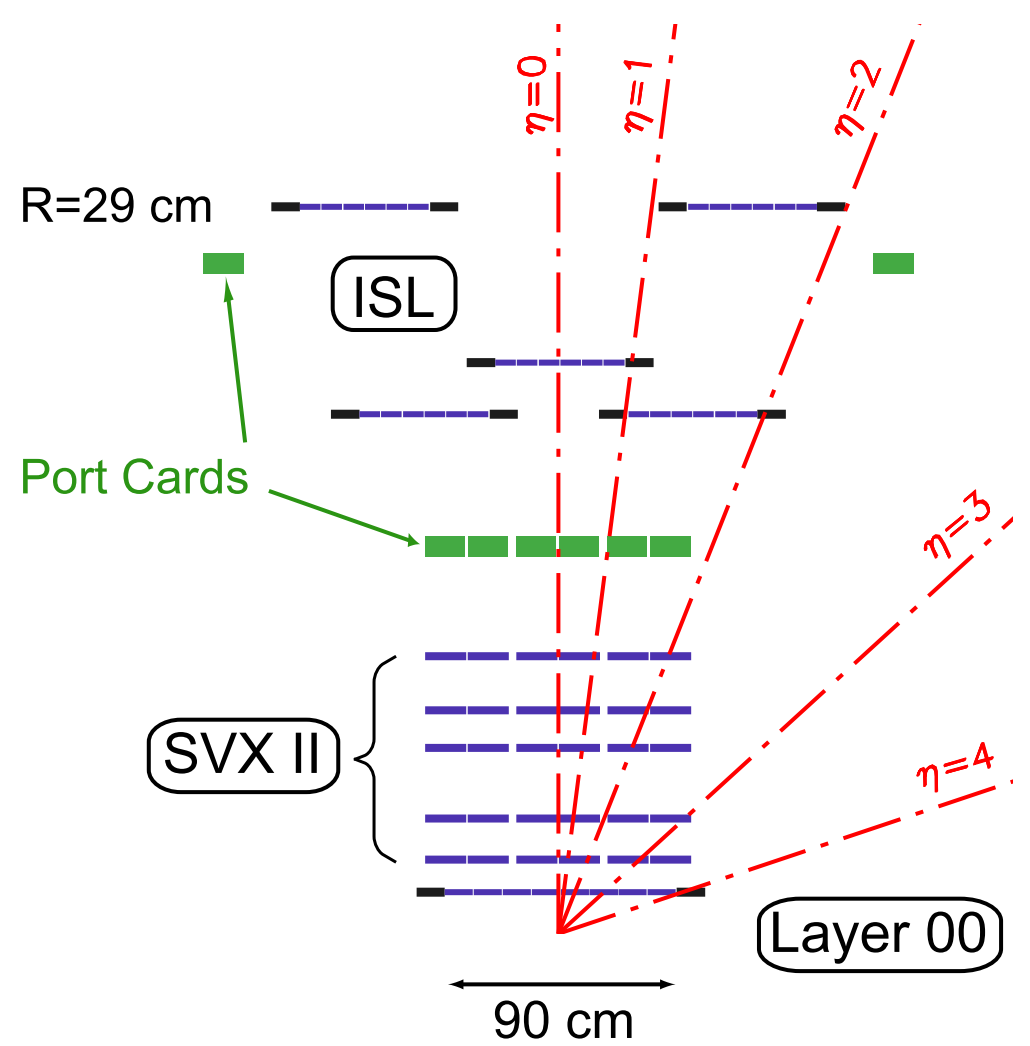

Figure 4.6.: A cross-sectional view of the silicon detector at CDF II showing the three sub-detector systems in the $r-z$ plane [19].

vapor-deposited gold on both sides. The COT chamber is filled with a nearly equal mixture of argon and ethane gas.

Charged particles produced in $p \bar{p}$ collisions travel through the COT and ionize the gas, producing electrons that drift along the electric field lines to the nearest sense wires. Collisions between the electrons and atoms of the gas in the high electric field region in close vicinity of the sense wire cause an avalanche multiplication of charges. The resulting charge is collected at the sense wire and the signal is detected through the read-out electronics. The tracking algorithms find charged particle trajectories that provide the best match to the observed pattern of hits on the sense wires. The hit position resolution is approximately $140 \mu \mathrm{m}$. The COT information for a charged particle can also be used in silicon reconstruction by matching the hits in the silicon 


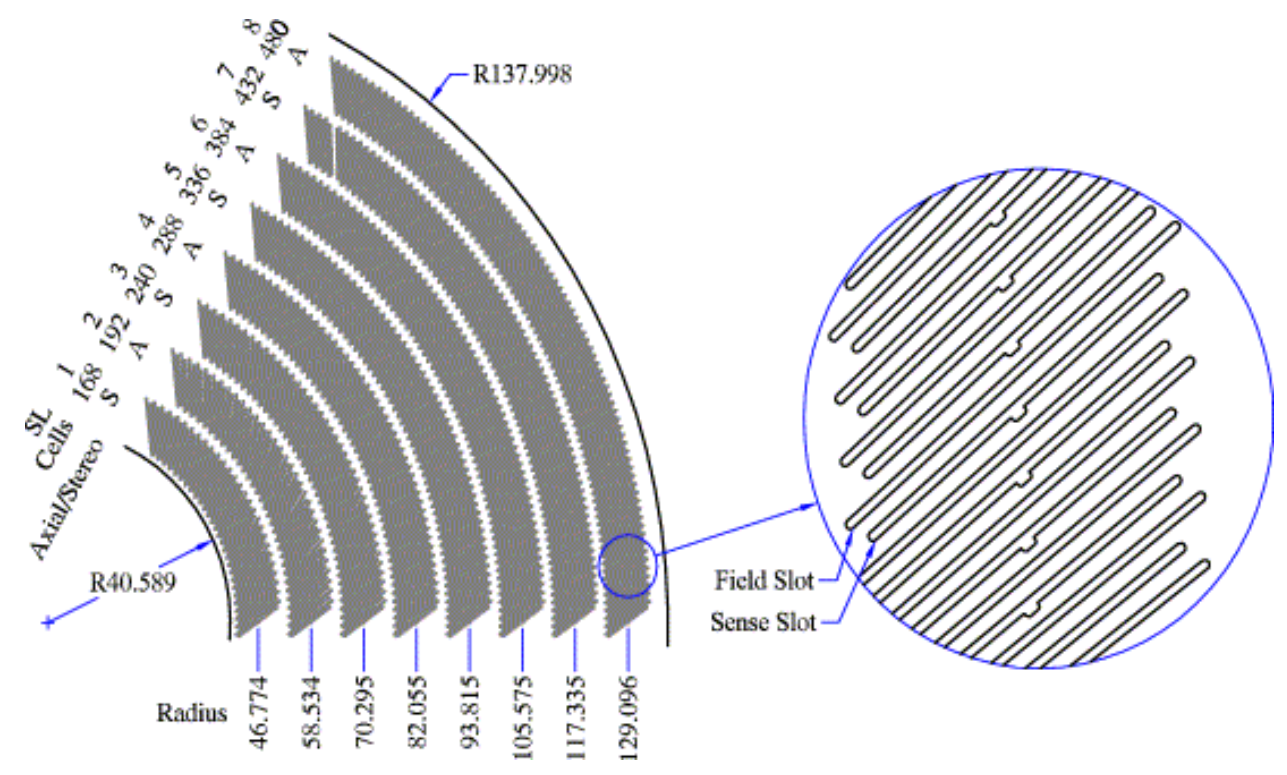

Figure 4.7.: Layout of the field sheet and sense wire planes in $1 / 6$ th section of the COT end plate [19].

detector to the pattern of hits on the sense wires in the COT. The set of hits or signals found in the COT, SVX, ISL and L00 detectors allow the determination of track trajectories using a variety of algorithms depending on the order in which the information from different tracking systems is processed. The track reconstruction efficiency is about $95 \%$ for tracks which pass through all the eight COT superlayers with $p_{T} \geq 400 \mathrm{MeV}$ and about $99 \%$ for those with $p_{T} \geq 2 \mathrm{GeV}$. This efficiency is largely dependent on the number of tracks in the chamber at a given time. Numerous closely spaced tracks can produce ambiguity among hits resulting in a failed track reconstruction.

The momentum of a charged particle produced in $p \bar{p}$ collisions is determined from the curvature, $C$, of its trajectories as it bends in the magnetic field inside the tracking chamber. The relation between the transverse momentum of a particle and its curvature in a magnetic field, $B_{z}$, is given by:

$$
p_{T}=\frac{A B_{z}}{|C|}
$$




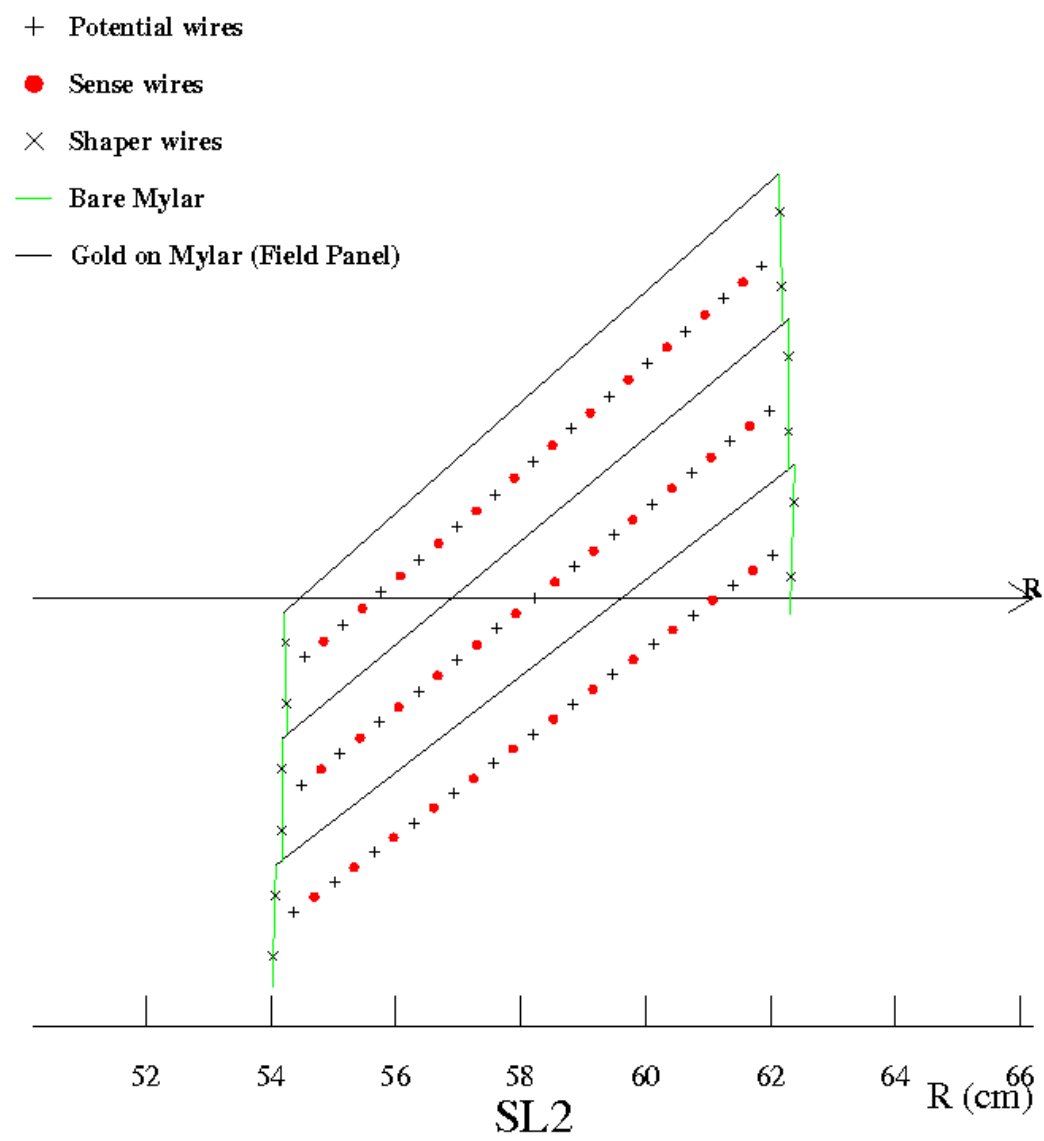

Figure 4.8.: Layout of wires in three supercells in $2^{\text {nd }}$ superlayer of the COT which runs along the beam direction [19].

where $A=1.5 \times 10^{-3} \mathrm{GeV} \mathrm{cm} / \mathrm{T}$. The transverse momentum resolution of the COT is about $\sigma_{p_{T}} / p_{T}^{2}=0.0015 \mathrm{GeV}^{-1}$.

Another important application of the COT is particle identification, using the ionization energy loss per unit track length $(d E / d x)$ of a particle as it travels through the detector. The ionization energy loss of a particle is related to its velocity and hence, the COT can be used for identifying charged particle species by extracting the particle's mass by combining velocity and momentum measurements. Identification 
of charged particles using $d E / d x$ information from the COT is an important aspect of the analysis presented in this dissertation and is discussed in detail in Chapter 5 .

\subsubsection{Time-of-Flight detector}

The Time-of-Flight (TOF) sub-detector is located between the tracking system and the cryostat for the solenoid magnet as shown in Figure 4.9. The TOF system is the primary detector system designed to distinguish between pions, kaons and protons with $p_{T} \leq 1.5 \mathrm{GeV} / \mathrm{c}$ by measuring the time it takes these particles to travel from the $p \bar{p}$ collision point to the TOF system. The detector is composed of 216 bars of

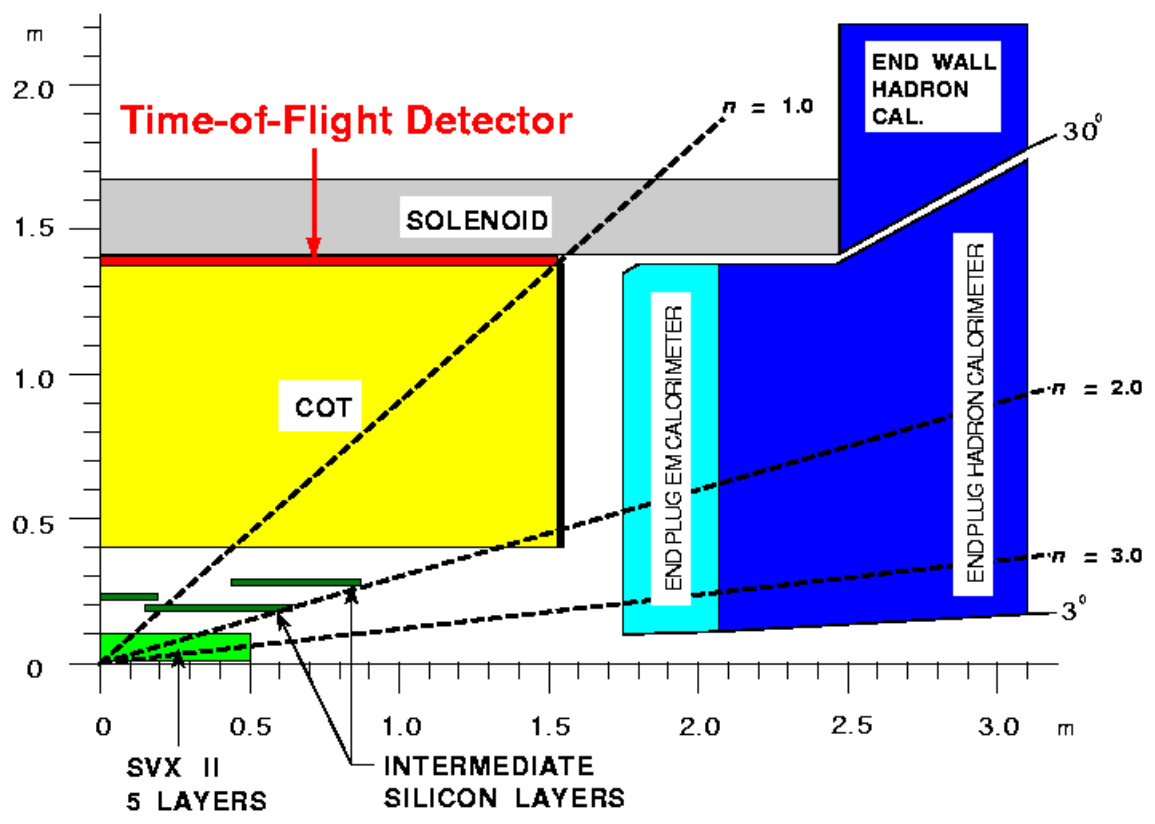

Figure 4.9.: Location of the Time-of-Flight system in the Collider Detector at Fermilab [19].

Bicron BC408 scintillator, each of which is $279.5 \mathrm{~cm}$ in length with a trapezoidal cross section of about $4 \mathrm{~cm} \times 4 \mathrm{~cm}[21]$. The bars are arranged in a cylindrical geometry at a radius of approximately $140 \mathrm{~cm}$ from the beam line, each covering $\sim 1.7^{\circ}$ in $\phi$ 
and pseudorapidity range $|\eta|<1$. A Hamamatsu R7761 19-stage fine-mesh photomultiplier tube (PMT) with a diameter of $1.5^{\prime \prime}$ is attached to each end of every bar.

Charged particles passing through the scintillator material produce photons that propagate towards the photo-multiplier tubes located at each end of the bar. The PMTs have a gain of $\sim 30000$ inside the $1.4 \mathrm{~T}$ magnetic field of the CDF II solenoid. The analog signal is processed by a pre-amplifier circuit mounted on each PMT before being passed to the read-out electronics, where the signal time and amplitude are digitized. The signal from each of the 432 channels follows two paths, one for the timing measurement and the second for a charge measurement.

The location of the bar where the signal is detected corresponds to the $\phi$ coordinate of the arrival point of the particle in the TOF system. The $z$ position of the signal in the bar can be determined by comparing the arrival times of the pulses recorded by the PMTs at each end of the bar. Although we do obtain some information about the $\phi$ and $z$ coordinates from TOF signals, they are measured more precisely in the COT. Hence, the $z$ position measured using the PMT hits in the TOF system must be consistent with the $z$ position determined from the COT. This is verified by extrapolating the particle trajectory in the COT to the TOF system and matching the trajectory with the $z$ position of the hits in the scintillator bar.

The combination of time-of-flight of a particle measured by TOF and $d E / d x$ information from the COT allows us to identify charged particles in practically all the $p_{T}$ regions accessible at CDF. Particle identification is a central part of our analysis since we measure the fraction of pions, kaons and protons produced produced in association with charm mesons. The details of the particle identification technique using the TOF sub-detector are discussed in Chapter 5.

\subsubsection{Calorimeters and Muon Chambers}

The information obtained from the Calorimeter systems and Muon Chambers does not have a significant contribution to the analysis presented in this dissertation. A 
brief description of these systems is presented in this section since they are important components of CDF and form an integral part of numerous other analysis performed using CDF data.

Calorimetry at CDF is used to distinguish electrons and photons from hadronic particles by measuring particle energy deposition. The calorimeter systems surround the solenoid magnet and are divided into two categories: Electromagnetic (EM) calorimeters which absorb the energy of electrons and photons via electromagnetic interactions and Hadronic calorimeters which stop long lived mesons and baryons by absorbing their energy via strong interactions with the nuclei in the detector material. The operational volume of the calorimeter systems is made up of layers of an absorbing metal material alternated with signal processing scintillator layers. In the EM calorimeter systems, lead is used as the absorber to induce bremsstrahlung in high energy electrons or electron-positron pair production by photons conversions. The resulting shower of secondary electrons and photons is detected by the PMTs attached by light guides to the scintillators and the signal strength is proportional to the energy of the incident particle. Iron is used as the absorbing material in the hadronic calorimeters, which induces hadronic showers via a variety of nuclear interactions. The secondary particles resulting from the hadronic interactions produce signals in the corresponding scintillators. Each sub-detector is segmented into towers in $\eta$ and $\phi$. The central electromagnetic (CEM) and central hadronic (CHA) calorimeters cover the pseudorapidity range $|\eta|<1$.1. Each tower in the central calorimeter system spans about 0.1 in $\eta$ and $15^{\circ}$ in $\phi$. In the higher $\eta$ regions, the plug calorimeter systems (PEM and PHA) extend the coverage to $|\eta|=3.6$. The towers in the plug calorimeter systems are arranged in variable segmentations ranging from 0.1 to 0.6 in $\eta$ and $7.5^{\circ}$ to $15^{\circ}$ in $\phi$. The wall hadronic system (WHA) bridges the gap between the central and plug hadronic components. Hadronic calorimeter towers are located directly behind the corresponding EM calorimeter towers and have matching segmentation. 
Muons are minimum ionizing particles and typically traverse and exit the CDF II detector before decaying without creating any showers in the calorimetry systems. The role of the muon systems it to reconstruct short tracks known as muon stubs at the outermost radii of the detector. A minimum of three muon hits is required for reconstructing a muon stub. Muon candidates are identified by extrapolating the reconstructed tracks from the inner tracking systems to the muon chambers, where they are tested for matches with muon stubs. In order to be considered as a match for a muon stub, the tracks in the COT are required to have minimum transverse momentum of $p_{T} \geq 1.4 \mathrm{GeV} / \mathrm{c}$. The CDF II detector contains four muon systems: the central muon (CMU), the central muon upgrade (CMP), the central muon extension (CMX) and the intermediate muon (IMU) detectors. The CMU is located outside the central hadronic calorimeter (CHA) and is segmented into 144 modules in $\phi$ each containing 4 layers of 4 rectangular drift cells. The sense wires are immersed in the same argon-ethane mixture used in the COT. It provides a coverage of $|\eta|<0.6$ and $p_{T}>1.4 \mathrm{GeV} / \mathrm{c}$. The CMP is an additional central muon detector located behind $60 \mathrm{~cm}$ thick steel shielding. It provides roughly the same coverage as the CMU and allows for cleaner muon identification due to the presence of steel absorbers. Since CMP is located behind thick steel shielding, a minimum $p_{T}$ of $2.2 \mathrm{GeV} / \mathrm{c}$ is required for the muons to reach the detector. The coverage of CMX detector is beyond the central muon systems, in the range $0.6 \leq|\eta| \leq 1.0$. It consists of a conical arrangement of drift cells within layers of scintillators. The CMX is azimuthally segmented in $15^{\circ}$ wedges each comprised of 8 layers of rectangular drift cells. The IMU consists of a barrel of drift chambers and scintillator counters which are similar to those of the other muon systems and provides a coverage of $1.0 \leq|\eta| \leq 1.5$ 


\subsubsection{Overview of the Trigger system at CDF}

The trigger system at CDF is essential since it is not physically feasible to store information about every single $p \bar{p}$ collision. At CDF, collisions occur roughly at a rate of $2.5 \mathrm{MHz}$, with the number of $p \bar{p}$ collisions occurring at every bunch crossing depending on the instantaneous luminosity delivered by the Tevatron. The data from the readout electronics of the full detector is referred to as an event, which has a size of approximately $250 \mathrm{kB}$. Hence, saving the full detector readout information for every event would be challenging in terms of both the bandwidth and storage capacity.

A trigger system is used, which is basically a filter to reduce the data rates and volumes to more manageable levels while selecting events that are likely to be useful for physics analyses. The data acquisition system can write up to 100 events per second to a buffer disk. The trigger system has to be fast enough to analyze every collision and decide which 100 events out of the total 2.5 million events should be recorded on tape every second. The trigger system is divided into three stages referred to as Level 1 (L1), Level 2 (L2) and Level 3 (L3). A schematic view of the data flow through the triggering system is shown in Figure 4.10.

The selection criteria at each level is more restrictive than the previous level reducing the event rate at every stage. Each level of the trigger has a certain maximum amount of time to decide whether a particular event is to be accepted or rejected. The time taken to make a decision may vary significantly from event to event. While the information about a current event is being processed, the detector readout information from other events is stored in the system. If the event is accepted, the information is sent to the higher level of the triggering system. Otherwise the information is overwritten by the data for the next event.

A particular set of criteria that an event is required to satisfy at L1, L2 and L3 defines the particular trigger path. The CDF II trigger system implements about 100 different trigger paths and an event is accepted if it satisfies the criteria for any one 


\section{Dataflow of CDF "Deadtimeless" Trigger and DAQ}

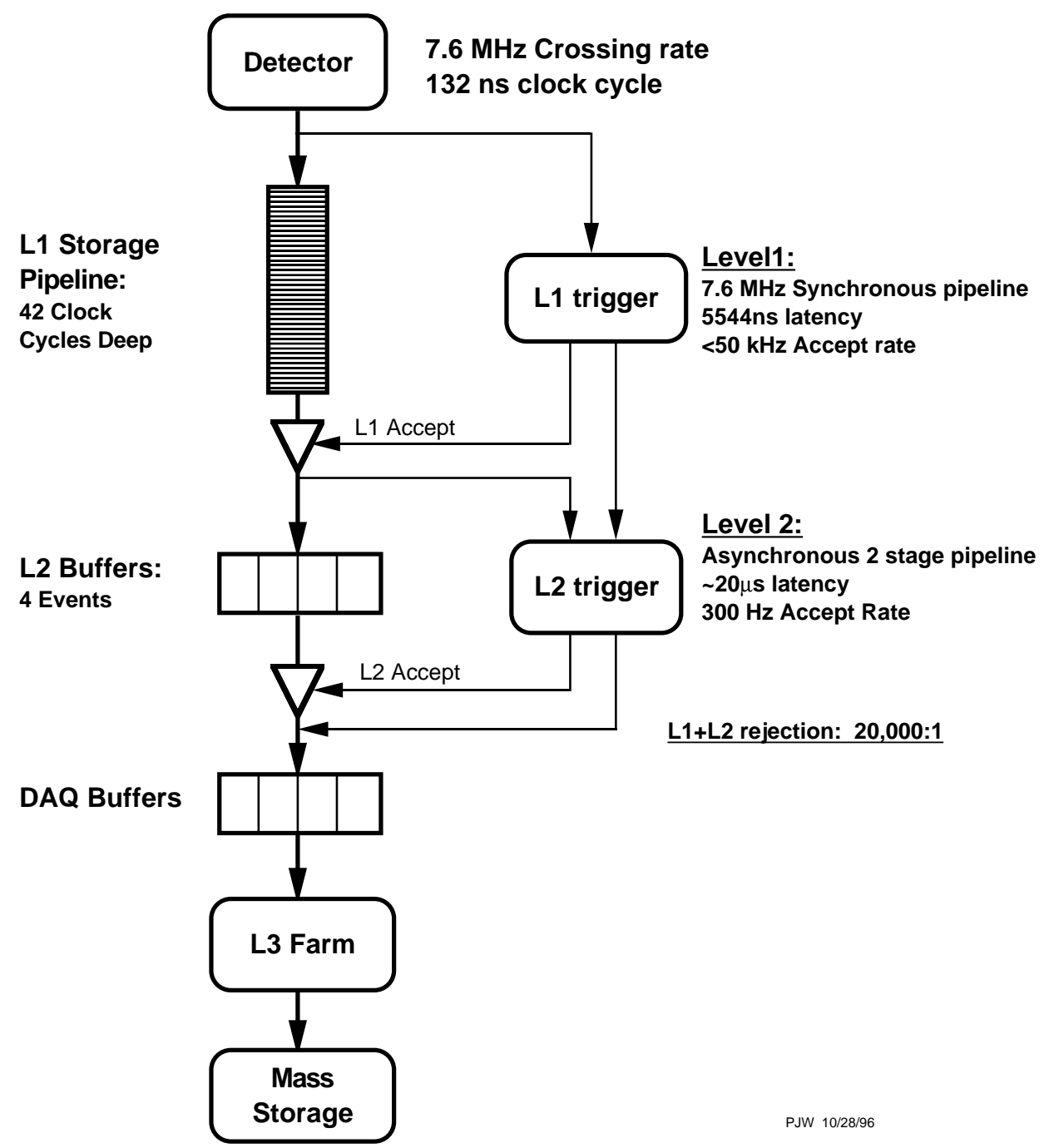

Figure 4.10.: A schematic diagram of the CDF II trigger system. The $p \bar{p}$ bunch crossing interval is $396 \mathrm{~ns}$ although the Tevatron and CDF II are designed to handle a bunch crossing interval of $132 \mathrm{~ns}$. The nominal data storage frequency is $100 \mathrm{~Hz}$ [19].

of the trigger paths. Figure 4.11 shows a block diagram of the various trigger paths implemented at L1 and L2. The trigger path that is used to select events for the 
analysis presented in this dissertation is the two track trigger described in detail in Section 4.2.8.

As the Tevatron luminosity decreases, the rate of events meeting a given trigger requirement is also reduced. However, the rate at which events are read and stored remains unchanged. If very strict requirements were enforced on the trigger paths such that they pass events at acceptable rates for high luminosities, the trigger bandwidth would become under-utilized as the luminosity decreases. On the other hand, loose trigger requirements would overwhelm the trigger system during high luminosities and good quality events could be missed as the system struggles to record all the events including the events which might be less interesting. A solution for efficient allocation of the trigger bandwidth is prescaling in which only every $N^{\text {th }}$ event that passes a trigger path may be recorded by the system. The prescale $N$ can be varied dynamically to achieve a desired acceptance rate, such that $N$ may be initially set high when the Tevatron luminosity is high and eventually decreased to unity as the luminosity decreases.

Level 1 (L1)

The first stage of the triggering system is the Level 1 trigger which operates at every bunch crossing. The L1 trigger is designed to process the events extremely fast and provides the highest rate reduction. It uses custom designed hardware to select events based on the information obtained from the COT, calorimeters and muon chambers. At Level 1, the track reconstruction is done by the eXtremely Fast Tracker (XFT). The XFT is capable of quickly reconstructing tracks in the COT by searching for matching pre-defined patterns of COT hits. This allows for very fast reconstruction of tracks with $p_{T}>1.5 \mathrm{GeV} / \mathrm{c}[22]$. The XFT system has very high efficiency of $\sim 96 \%$, good transverse momentum resolution $\delta p_{T} / p_{T}=0.016 p_{T}$ and good azimuthal resolution $\delta \phi \sim 5 \mathrm{mrad}$. The tracks found by the XFT are passed to the Extrapolation System (XTRP) which processes and distributes the tracking 
information to other elements of the Level 1 triggering system. The XTRP matches tracks with the signals found in the calorimeter systems to identify electrons and with stubs found in the muon chambers to identify muons. All the Level 1 information is sent to the Global Level 1 system (see Figure 4.11) for processing where the event selected if it satisfies one of the various Level 1 trigger paths. The Global Level 1 has a capacity to accommodate information for about 64 different Level 1 trigger paths. Data from an event accepted at L1 is moved to one of the four L2 buffers.

Level 2 (L2)

The Level 2 trigger system reduces the Level 1 accept rate of approximately $25 \mathrm{kHz}$ to about $500 \mathrm{~Hz}$. At this stage, the information available from the Level 1 trigger is supplemented with information from the silicon detectors, the calorimeters and the muon chambers. The Silicon Vertex Trigger (SVT) [23] system used at L2 is most important to the analysis presented in this dissertation as it allows measurement of the track impact parameter $d_{0}$. The SVT combines the coarse tracking information from the XFT with high precision information about the hits in the SVX-II detector. In addition to providing precise information about the track impact parameter, the second level trigger system also improves the measurement of $p_{T}$ and $\phi_{0}$. The SVT works by extrapolating the XFT tracks into the volume of the SVX-II detector and matching them to the $r-\phi$ hits in the SVX-II. The information about the hits in the SVX detector and the XFT segments is fed into a fitter which returns the measurements on $p_{T}, \phi_{0}$ and $d_{0}$ of the track. The impact parameter information obtained from the SVT forms the basis of many heavy flavor trigger paths which require at least one displaced track with non-zero $d_{0}$ in the event.

Level 3 (L3)

The last stage of the CDF II triggering system is the Level 3 trigger. Level 3 is implemented as a software trigger which uses a PC farm with about 500 CPU's, each 
of which provides a processing slot for one event. Since the input rate for the Level 3 system is about $500 \mathrm{~Hz}$, this allocates about one second for event reconstruction and to reach a trigger decision. When an event is accepted by the Level 2 trigger, its data becomes available for readout distributed over a large number of VME Readout Buffers (VRBs). A system called the Event Builder assembles all these small pieces of data in a data structure called the event record and sends them to one place in the Level 3 farm where the final level of event filtering is performed. The Level 3 trigger performs a full reconstruction of the event in the detector, building high level objects corresponding to charged particle such as kaon, pion tracks and leptons, using the full resolution of the detector components. Events that satisfy the Level 3 selection requirements are stored on disk and subsequently written on tape at a rate of about $50 \mathrm{~Hz}[24]$. The data saved on tape is finally used for the various offline physics analyses.

\subsubsection{The two track trigger}

The analysis presented in this dissertation involves analyzing events that satisfy the two track trigger path criteria. The tracks corresponding to the decay products of charm mesons often have a large impact parameter with respect to the primary vertex (collision point). This signature could lead to the selection of an event with a charm meson decay by the SVT system, provided the event also satisfies all the other triggering criteria. The two track trigger attempts to identify a set of two displaced tracks in the event based on the transverse momentum $p_{T}$, impact parameter $d_{0}$, opening angle between the two tracks in the transverse plane $\Delta \phi$ and transverse

decay length $L_{x y}$ of the two track vertex. The set of two trigger tracks can be a combination of a generic track in the event and a decay product of the charm meson or both trigger tracks could be decay products of the charm meson.

The two track trigger is composed of many trigger paths that are categorized into three scenarios, which depend on the instantaneous luminosity of the Tevatron. 
These scenarios are referred to as Scenario Low, Scenario A and Scenario C, which have different requirements for the minimum $p_{T}$ of a track and minimum sum $p_{T}$ the track pair [25]. Scenario A is the nominal two trigger path and is prescaled at high luminosity. The unprescaled Scenario $\mathrm{C}$ path imposes more stringent requirements to ensure the acceptance of good quality events at higher luminosities. Scenario Low is a variation of Scenario A that is designed to accept a more inclusive sample of events at low luminosities.

The trigger paths have minimum requirements for $p_{T}, \Delta \phi, L_{x y}$ and $d_{0}$ at each level of the triggering system. The selection criteria for each of the above mentioned quantities measured at L1-XFT, L2-SVT and L3, for all the three two track trigger scenarios is listed below:

\section{Scenario A}

1. Level 1

- Two XFT tracks with opposite charge

- $p_{T}>2.0 \mathrm{GeV} / \mathrm{c}$ for each XFT track

- $\sum p_{T}>5.5 \mathrm{GeV} / \mathrm{c}$

- $0^{\circ}<\Delta \phi_{6}<135^{\circ}$, the separation between a pair of tracks in $\phi$ as measured by the XFT at the radius of superlayer 6 in the COT

2. Level 2

- Both XFT tracks matched to an SVT track

- $p_{T}>2.0 \mathrm{GeV} / \mathrm{c}$ for each SVT track

- $\sum p_{T}>5.5 \mathrm{GeV} / \mathrm{c}$

- $2^{\circ}<\Delta \phi<90^{\circ}$

- $120 \mu \mathrm{m} \leq\left|d_{0}\right| \leq 1 \mathrm{~mm}$ for each SVT track

- $L_{x y}>200 \mu \mathrm{m}$ for the two track vertex 
3. Level 3

- SVT tracks matched to COT+silicon tracks

- $p_{T}>2.0 \mathrm{GeV} / \mathrm{c}$ for each $\mathrm{COT}+$ silicon track

- $\sum p_{T}>5.5 \mathrm{GeV} / \mathrm{c}$

- $2^{\circ}<\Delta \phi<90^{\circ}$

- $80 \mu \mathrm{m} \leq\left|d_{0}\right| \leq 1 \mathrm{~mm}$ for each COT+silicon track

- $\Delta z_{0}<5 \mathrm{~cm}$

- $L_{x y}>200 \mu \mathrm{m}$ for the two track vertex

\section{Scenario C}

1. Level 1

- Two XFT tracks with opposite charge

- $p_{T}>2.5 \mathrm{GeV} / \mathrm{c}$ for each XFT track

- $\sum p_{T}>6.5 \mathrm{GeV} / \mathrm{c}$

- $0^{\circ}<\Delta \phi_{6}<120^{\circ}$

2. Level 2

- Both XFT tracks matched to an SVT track

- $p_{T}>2.5 \mathrm{GeV} / \mathrm{c}$ for each SVT track

- $\sum p_{T}>6.5 \mathrm{GeV} / \mathrm{c}$

- $2^{\circ}<\Delta \phi<90^{\circ}$

- $120 \mu \mathrm{m} \leq\left|d_{0}\right| \leq 1 \mathrm{~mm}$ for each SVT track

- $L_{x y}>200 \mu \mathrm{m}$ for the two track vertex

3. Level 3 
- SVT tracks matched to COT+silicon tracks

- $p_{T}>2.5 \mathrm{GeV} / \mathrm{c}$ for each $\mathrm{COT}+$ silicon track

- $\sum p_{T}>6.5 \mathrm{GeV} / \mathrm{c}$

- $2^{\circ}<\Delta \phi<90^{\circ}$

- $80 \mu \mathrm{m} \leq\left|d_{0}\right| \leq 1 \mathrm{~mm}$ for each COT+silicon track

- $\Delta z_{0}<5 \mathrm{~cm}$

- $L_{x y}>200 \mu \mathrm{m}$ for the two track vertex

\section{Scenario Low}

1. Level 1

- Two XFT tracks without opposite charge

- $p_{T}>2.0 \mathrm{GeV} / \mathrm{c}$ for each XFT track

- $0^{\circ}<\Delta \phi_{6}<90^{\circ}$

2. Level 2

- Both XFT tracks matched to an SVT track

- $p_{T}>2.0 \mathrm{GeV} / \mathrm{c}$ for each SVT track

- $2^{\circ}<\Delta \phi<90^{\circ}$

- $120 \mu \mathrm{m} \leq\left|d_{0}\right| \leq 1 \mathrm{~mm}$ for each SVT track

- $L_{x y}>200 \mu \mathrm{m}$ for the two track vertex

3. Level 3

- SVT tracks matched to COT+silicon tracks

- $p_{T}>2.0 \mathrm{GeV} / \mathrm{c}$ for each $\mathrm{COT}+$ silicon track

- $2^{\circ}<\Delta \phi<90^{\circ}$ 
- $80 \mu \mathrm{m} \leq\left|d_{0}\right| \leq 1 \mathrm{~mm}$ for each COT+silicon track

- $\Delta z_{0}<5 \mathrm{~cm}$

- $L_{x y}>200 \mu \mathrm{m}$ for the two track vertex

\subsubsection{Computing and Software}

The events accepted by the CDF II trigger system that are stored on tape are not optimal for usage in physics analyses because the latest calibration information of the CDF II detector systems is not usually available for online event reconstruction.

The complete event reconstruction process involves handling large volumes of complex, structured data which describe the observed physical interactions, components of the detector and the latest calibration information used to interpret the data. In order to maintain an adequate record of detector performance, detailed information on calibrations, detector status and beam conditions is recorded during running, all of which is stored in a database. In the reconstruction process, readout data from various detector components is combined using algorithms to generate higher level structures such as tracks and leptons. An example of this process is the assembling of hit data from the tracking chamber into track segments which are further assembled into track helix parameters.

The event reconstruction package is composed of a number of independent modules, each performing a specific task, that execute sequentially in a specific order. For example, the tracking module must be run before the electron finding module because the electron identification module requires tracks. The event processing is carried out on production farms of PC's connected to a central I/O server. PC farms provide a cost effective way to obtain the necessary computing power required to perform the complete event reconstruction. Each event is read from tape or disk and sent to a worker node for processing. When processing is complete, the event is sent back to the I/O server for output. At this stage, the data is also split into different physics datasets based on the trigger paths that were satisfied by the processed event. 
Events from the hadronic dataset hbhd0d were used for the analysis presented in this dissertation. 


\section{RUN II TRIGGER SYSTEM}

Detector Elements

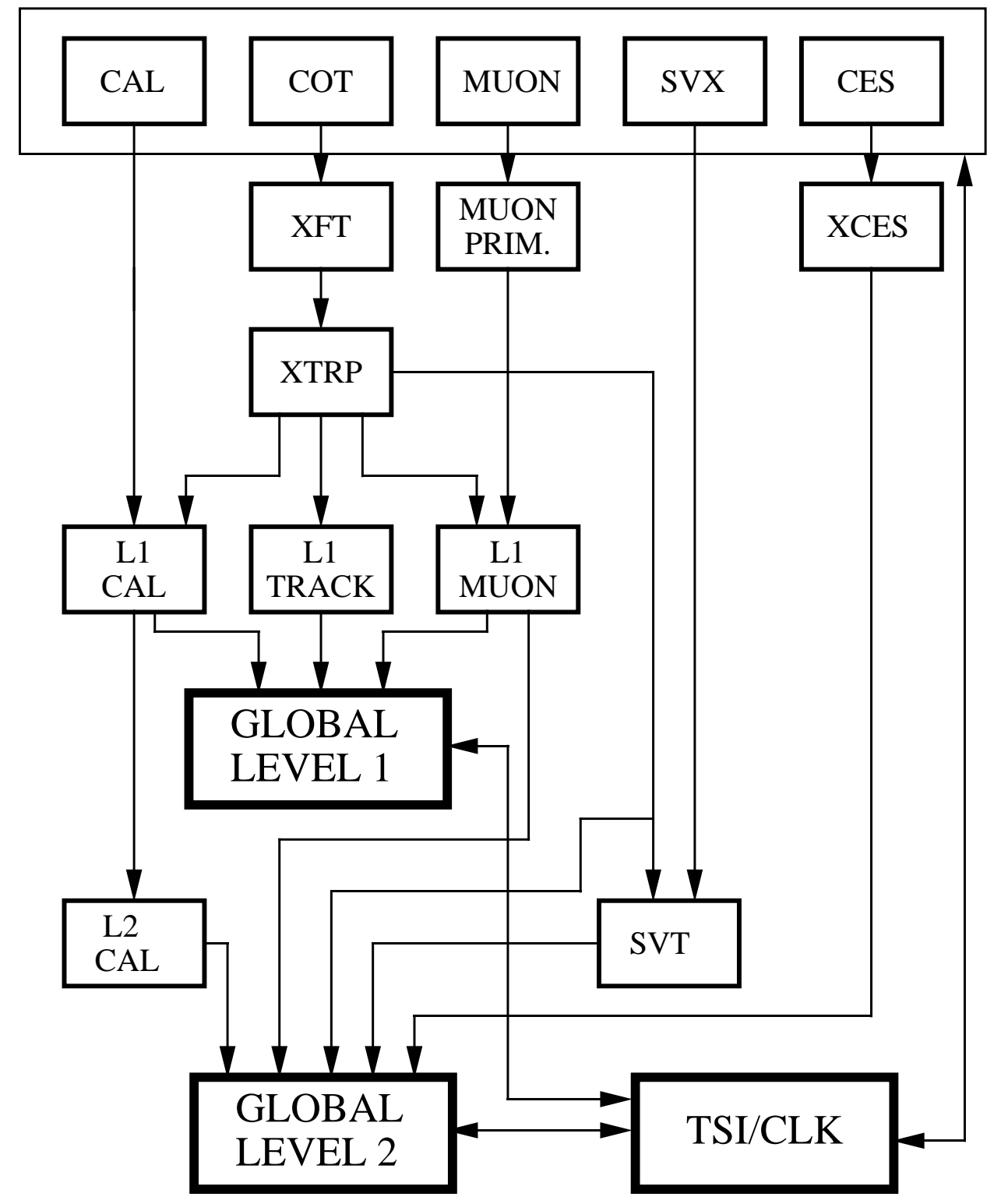

PJW 9/23/96

Figure 4.11.: Block diagram illustrating the flow of information from the CDF II detector elements through the different components of the trigger system [19]. 


\section{PARTICLE IDENTIFICATION TECHNIQUES}

The measurements of branching fractions of $\mathrm{B}, \mathrm{D}$ and $\tau$ decays have played an important role in our understanding of the electroweak sector. Many of these measurements involve studying exclusive decay modes that utilize various particle identification techniques for identifying charged decay products such as kaons, pions, electrons and protons. For example, particle identification techniques at CDF were extensively used in the measurement of branching fractions such as $\operatorname{BR}\left(B^{+} \rightarrow \phi K^{+}\right)[26], \operatorname{BR}\left(\bar{B}_{s}^{0} \rightarrow\right.$ $\left.D_{s}^{+} K^{-}\right) / \operatorname{BR}\left(\bar{B}_{s}^{0} \rightarrow D_{s}^{+} \pi^{-}\right)[27]$ and $\operatorname{BR}\left(B^{-} \rightarrow D^{0} K^{-}\right) / \operatorname{BR}\left(B^{-} \rightarrow D^{0} \pi^{-}\right)$[28], which involved identifying the charged kaon or pion track. Using particle identification techniques is an essential component of the analysis presented in this dissertation since the goal is to identify charged kaons produced in association with $D_{s}^{ \pm} / D^{ \pm}$mesons.

The analysis described in this dissertation involves reconstructing hadronic decays and focuses mainly on the identification of kaons, pions and protons. We use two techniques for charged particle identification, namely, the measurement of the specific ionization per unit track length $(d E / d x)$ in the Central Outer Tracker and the time of flight of the particle measured in the Time-of-Flight sub-detector.

\subsection{Particle identification using Time-of-Flight}

The CDF-II Time-of-Flight detector is described in detail in Section 4.2.5. Particle identification using the TOF sub-detector is performed by measuring the arrival time of a particle at a TOF scintillator bar with respect to the $p \bar{p}$ collision time, $t_{0}$ [29]. The mass, $m$, of a particle can be determined from the momentum, $p$, path length, $L$ measured in the tracker, and the time-of-flight, $t$, using the equation:

$$
m=\frac{p}{c} \sqrt{\frac{c^{2} t^{2}}{L^{2}}-1}
$$


where $p$ and $L$ are measured by the tracking system. The $p \bar{p}$ interaction region has a longitudinal spread of $\sim 30 \mathrm{~cm}$, which results in interactions being spread out in time by approximately $1-1.5 \mathrm{~ns}$, which is large compared to the expected TOF resolution of $\sim 100 \mathrm{ps}$. For this reason, the production time $t_{0}$ is determined on an event-by-event basis by performing a likelihood fit including all tracks in each event. For each track that hits the TOF detector, $t_{0}$ is calculated assuming three mass hypotheses, namely kaon, pion and proton. Then, the $t_{0}$ of the given event is obtained by performing a likelihood fit to the $t_{0}$ distribution of all tracks in the event, assuming the following composition of tracks: $80 \%$ pions, $10 \%$ kaons and 10\% protons [29]. The above procedure results in a typical $t_{0}$ resolution that is less than $100 \mathrm{ps}$.

\subsubsection{TOF residual distribution}

The time that a given particle takes to reach the TOF detector can be calculated using the momentum and trajectory of the particle and an assumed mass hypothesis using:

$$
t=\frac{L}{c} \sqrt{\frac{m^{2} c^{2}}{p^{2}}+1}
$$

Using the arrival time of a particle at TOF $\left(t_{t o f}\right)$ and the time of flight, the predicted production time $\left(t_{\text {pred }}\right)$ of the particle can be calculated $t_{\text {pred }}=t_{\text {tof }}-t_{\text {flight }}$. The distribution of the difference in the predicted and measured production time of a particle, $t_{\text {pred }}-t_{0}$, is referred to as the TOF residual distribution.

The procedure for estimating the $t_{0}$ of a given track is the same as the procedure used to estimate the $t_{0}$ of an event, except that while determining the production time $t_{0}$ for a given track, it is excluded from the $t_{0}$ likelihood fit, which is instead performed including all other tracks in the event [30]. The given track is removed from the fit so that an unbiased comparison can be made between the $p \bar{p}$ interaction time and the measured time of TOF hits associated with the track. 
The TOF residual distribution can be used to identify various particle types as follows. For a correct mass hypothesis, the $t_{\text {pred }}-t_{0}$ distribution will be centered at zero and the width of the distribution will give the timing resolution of the TOF detector. An incorrect mass hypothesis shifts the residual distribution by an amount depending on the particle momentum and mass.

For example, if we have a sample of kaons, pions and protons with similar momenta and assume a pion mass hypothesis, the distribution $t_{\text {pred }}(\pi)-t_{0}$ will consist of several peaks corresponding to the different particles species. Under the pion hypothesis, the peak corresponding to the pion component in the sample will be centered at zero whereas the kaon and proton peaks will be shifted with respect to the pion peak. Each peak in the TOF residual distribution will be centered at a value $t_{\text {pred }}(\pi)-t_{\text {pred }}(i)$, where $i$ refers to the particle type.

The actual position of the mean of the peaks corresponding to different particles types with similar momenta in the residual distribution will depend on the assumed particle hypothesis. For example, under a kaon mass hypothesis, the kaon peak will be centered at zero, whereas the pion and proton peaks will be shifted. We assume that the $t_{\text {pred }}(i)-t_{0}$ distribution for all particle types has the same shape because for a correct mass hypothesis the width of the distribution essentially denotes the resolution of the TOF detector, which is expected to be independent of the particle type.

\subsubsection{Calibrating the TOF residual distribution}

Since the response of the TOF detector is expected to be independent of particle type, the shape of the TOF residual distribution was calibrated using a pure sample of either kaons, pions and protons. In the analysis presented in this dissertation, a sample of pions from the hadronic decay mode $D^{* \pm} \rightarrow D^{0} \pi^{ \pm}$is used to calibrate the residual distribution. 
We reconstruct $D^{*+} \rightarrow D^{0} \pi^{+}, D^{0} \rightarrow K^{-} \pi^{+}$and the charge conjugate decays using approximately $360 \mathrm{pb}^{-1}$ of the same data set that is used for the charm fragmentation analysis. The reconstructed $K \pi \pi$ candidates are required to satisfy the Two Track Trigger described in Section 4.2.8. We use the $\Delta m=m(K \pi \pi)-m(K \pi)$ invariant mass difference distribution in an un-binned likelihood fit to separate the signal and combinatorial background components in the sample. The decay channel provides a pure sample of pions in the signal region above the background component that is used to calibrate the shape of the time of flight residual distribution.

In order to extract the optimal parameterization of the time of flight residual distribution, we perform a combined un-binned likelihood fit using the $\Delta m$ distribution and the time of flight residual distribution of the pion track. The probability density functions used to describe the shape of the various components in the likelihood fit are listed below:

- Signal component in the $\Delta m$ distribution: sum of two Gaussian functions with different widths but the same mean position.

- Background component in the $\Delta m$ distribution: the shape of the background component is described by the following empirical function which is normalized in the mass range $\left[m_{\pi}, m_{\max }\right]$ :

$$
b(\Delta m)=\frac{B^{c+1} e^{-B\left(\Delta m-m_{\pi}\right)}\left(\Delta m-m_{\pi}\right)^{c}}{\Gamma(c+1) P\left(c+1, B\left(m_{\max }-m_{\pi}\right)\right)}
$$

where $m_{\max }=0.17 \mathrm{GeV} / \mathrm{c}^{2}$. The values of the parameters $B$ and $c$ are obtained from the likelihood fit.

- Signal peak(s) in the TOF residual $\left(t_{r}\right)$ distribution: sum of two Gaussian functions with different widths but the same mean position (TOF signal resolution function).

- Tails in the TOF residual distribution: using the following empirical function

$$
T(x)=\frac{(|x| / b)^{q} e^{(-a|x| / b)}}{b \Gamma(q+1) / a^{q+1}}
$$


where the values of the parameters $b, a$ and $q$ are obtained from the likelihood fit. This functional form is a good choice to describe the tails since the value of $T(x)$ is zero at $x=0$ and rises on both sides of the origin before finally falling exponentially. Hence, the shape is very distinct from the TOF signal resolution function that peaks at zero and describes the bulk of the TOF residual distribution.

The likelihood function for the combined $\left(\Delta m, t_{r}\right)$ likelihood fit is shown in the equation:

$$
F\left(\Delta m, t_{r}\right)=f_{s} F_{s}(\Delta m)\left[\sum_{i=\pi, K, p} f_{i}^{s} F_{i}\left(t_{r}\right)\right]+\left(1-f_{s}\right) F_{b}(\Delta m)\left[\sum_{j=\pi, K, p} f_{j}^{b} F_{j}\left(t_{r}\right)\right]
$$

where $f_{s}$ denotes the signal fraction in the sample. The notations $F_{s}(\Delta m)$ and $F_{b}(\Delta m)$ represent to the probability density functions used to describe the shape of the signal and background components in the $\Delta m$ distribution respectively. The probability density function used to describe the shape of the TOF residual distribution is denoted by $F\left(t_{r}\right)$, which is a sum of the TOF signal resolution function and the contribution from the tails. The notation $f_{i}^{s}$ denotes the fraction of particle type $i$ in the signal region, where $i \in\{\pi, K, p\}$. Similarly, $f_{j}^{b}$ denotes the fraction of particle type $j$ in the background region, where $j \in\{\pi, K, p\}$.

The combined likelihood fit is performed in ranges of transverse momentum $p_{T}$ of the pion track. Figure 5.1 shows the invariant mass difference distribution and the time of flight residual distribution (of the pion track) in the lowest $p_{T}(\pi)$ range. The projections of the probability density functions obtained from the combined likelihood fit are also shown in the figure. We observe similar level of agreement in the other transverse momentum ranges in which the likelihood fit is performed [31].

The left hand side plot in Figure 5.1 shows a clean signal peak in the invariant mass difference distribution, which consists entirely of pions. These pure pions in the signal region constitute the large pion peak in the time of flight residual distribution, shown in the right hand side plot, that is centered at zero since the plot is made assuming the pion mass hypothesis. The kaon and proton peaks that are shifted with respect 
to the pion peak represent the kaons and protons in the small background component underneath the signal region. The pion peak can also have a small component from pions in the background region in addition to the pure pion sample constituting the signal.

We conducted test studies to assess whether the parametrization of the time of flight residual distribution obtained from the pion sample induces any bias in identifying different particle types. These studies involve testing the combined particle identification techniques, including both TOF and $d E / d x$ information, and are described in Section 5.4 . 

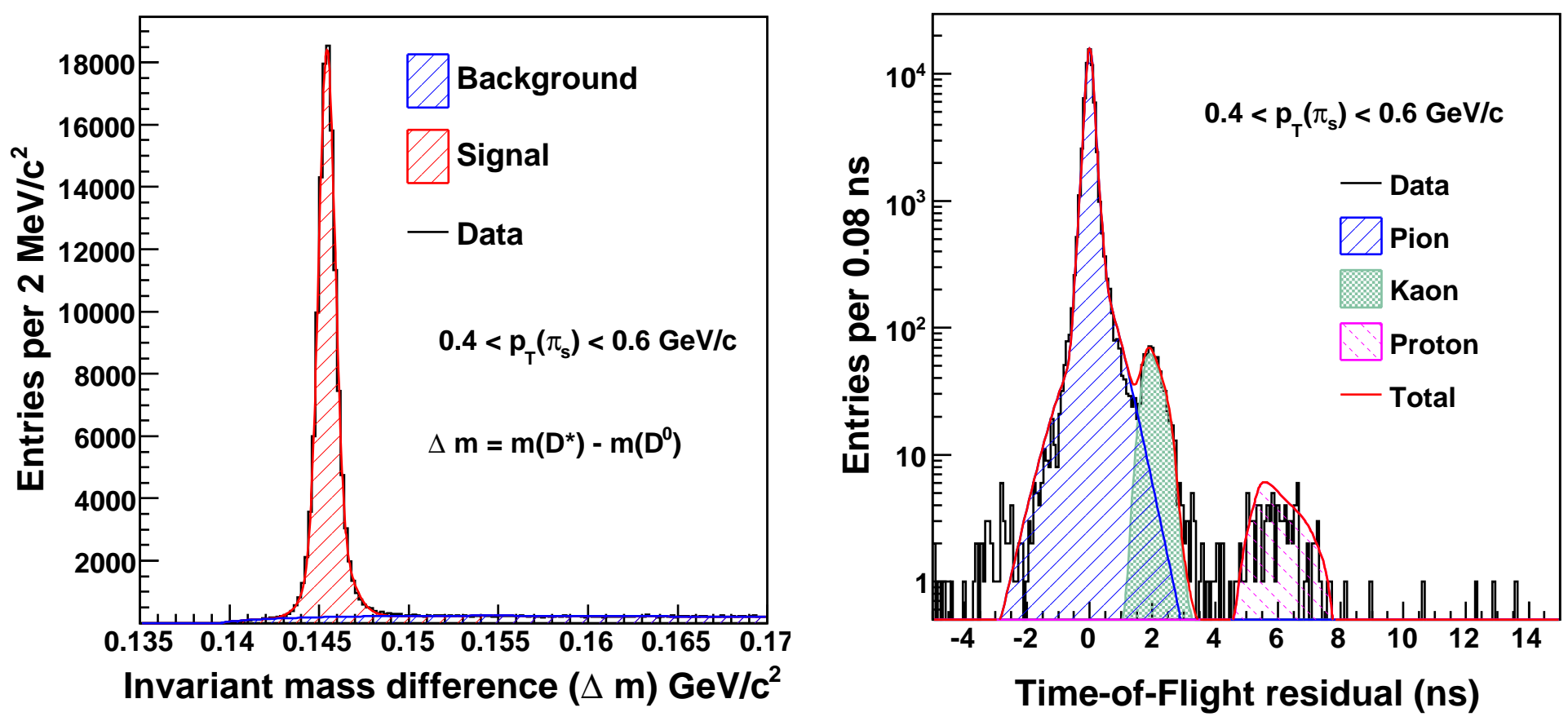

Figure 5.1.: (1) Invariant mass difference distribution, $\Delta m=m(K \pi \pi)-m(K \pi)$, for the reconstructed $K \pi \pi$ candidates $(2)$ TOF residual distribution for the pion track, assuming the pion mass hypothesis. The data corresponds to a momentum range of $0.4<p_{T}(\pi)<0.45 \mathrm{GeV} / \mathrm{c}$. 


\subsection{Particle identification using $d E / d x$ measured in the COT}

When a charged particle travels through a gas, it leaves a trail of ionization along its flight path. The average total energy loss per unit length of a particle with charge $q$ and velocity $\beta c$ is described by the Bethe-Bloch formula [32]:

$$
\left\langle\frac{d E}{d x}\right\rangle=\frac{4 \pi N e^{4}}{m c^{2} \beta^{2}} q^{2}\left[\log \left(\frac{2 m c^{2} \beta^{2} \gamma^{2}}{I^{2}}\right)-\beta^{2}-\frac{\delta(\beta \gamma)}{2}\right]
$$

where $N$ is the number density of electrons in the medium, $m$ is the mass of the electron, $e$ is the charge of the electron and $I$ is the mean excitation energy of the atoms in the medium. Equation 5.6 shows that for a drift chamber with fixed gas properties, $d E / d x$ depends only on the velocity $(\beta c)$ for a charged particle. Therefore, when combined with a measurement of the particle momentum, $d E / d x$ can be used to identify particles of different mass. Stable particles like electrons, muons, pions, kaons and protons exhibit different $d E / d x$ depending on their momentum as shown in Figure 5.2. At CDF, the gas in the tracker is kept at a pressure of $1 \mathrm{~atm}$, which is much lower than the pressures kept in drift chambers that are optimized for $d E / d x$ measurements as shown in Figure 5.2. Nevertheless, a good calibration of the $d E / d x$ response can provide some separation between various particle species, which is important in the momentum ranges where the separation power provided by TOF falls below $1 \sigma$. The separation power of the $d E / d x$ and TOF techniques is discussed in Section 5.3.

A detailed description of the design of the CDF-II tracker is presented in Section 4.2.4. The sense wires in the COT collect the charge produced by the ionizing incident charged particle, which is read out by the Amplifier-Shaper-DiscriminatorCharge (ASDQ) front-end electronics. The ASDQ is a custom Application-Specific Integrated Circuit (ASIC) designed specifically for the COT. The input analog signal is processed by ASDQ and the output is a digital pulse. The leading edge gives the arrival time information and the pulse width is related to the amount of charge collected by a sense wire. For a given particle, energy loss measurements can be obtained from multiple sense wires but the highest $20 \%$ of the measured charge values are discarded. 

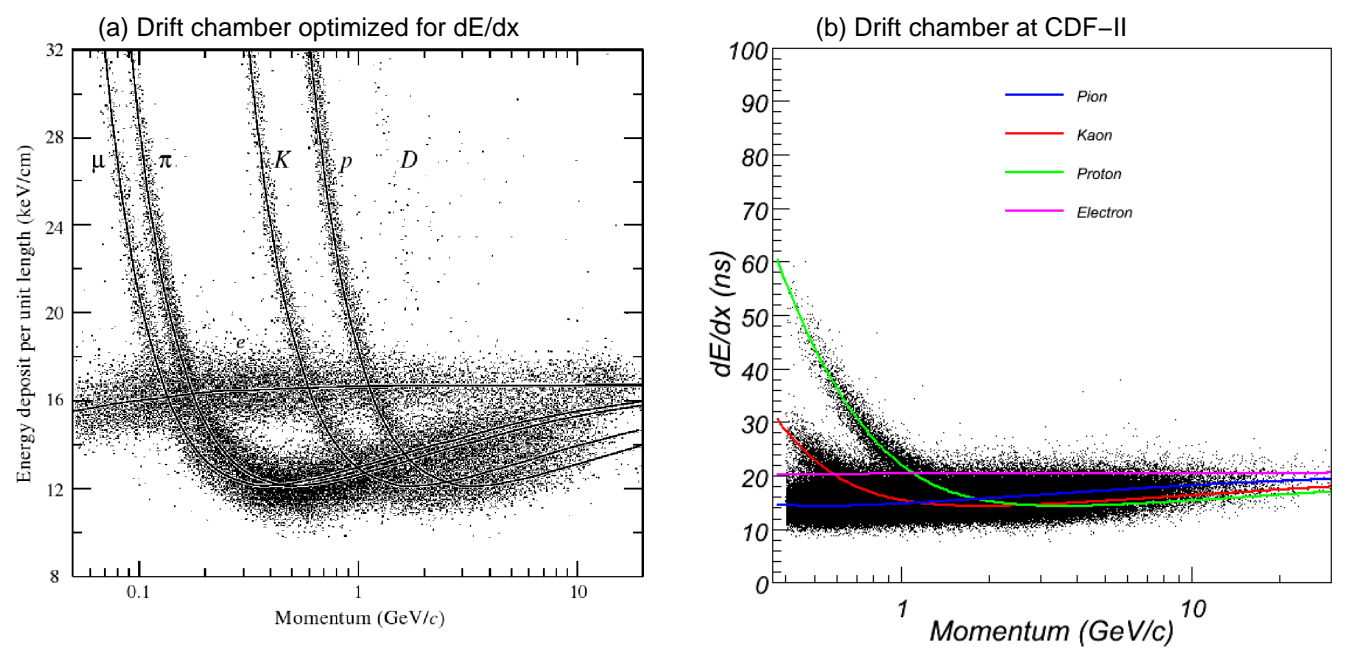

Figure 5.2.: Energy loss per unit length $d E / d x$ for various particles traversing a drift chamber: (a) Response of a drift chamber filled with gas at a pressure of $8.5 \mathrm{~atm}$, optimized for $d E / d x$. (b) Response of the CDF-II drift chamber that is not particularly optimized for $d E / d x$ measurements.

The $80 \%$ truncated mean is taken as the best estimate of the track $d E / d x$ because it is less sensitive to large Landau fluctuations in the deposited ionization. In the case of the tracker at CDF-II, the following empirical variant of the Bethe-Bloch equation better models the average energy loss of a particle:

$$
\left\langle\frac{d E}{d x}\right\rangle=\frac{1}{\beta^{2}}\left(c_{1} \log \frac{\beta \gamma}{\beta \gamma+b}+c_{0}\right)+a_{1}(\beta-1)+a_{2}(\beta-1)^{2}+c
$$

The values of the parameters $c_{1}, c_{0}, b, a_{1}, a_{2}$ and $c$ describing the shape of the universal curve are obtained by using the distribution of measured $d E / d x$ in the data.

\subsubsection{Calibrating the COT $d E / d x$ response}

In order to calibrate the $d E / d x$ response of the tracker, we study a sample of tracks produced in the vicinity of a $D^{* \pm} \rightarrow D^{0} \pi^{ \pm}$decay. We choose a generic sample of 
tracks around $D^{* \pm}$ decays because these generic tracks have the same environment as the sample of tracks used for the $D_{s}^{ \pm} / D^{ \pm}$fragmentation study. Since the generic track sample used for the $d E / d x$ calibration contains a mixture of kaons, pions, protons and electrons, we use their TOF residual distribution as additional information for identifying the particles in the generic sample.

In order to obtain the optimal values of the parameters describing the shape of the universal curve in Equation 5.7, an un-binned likelihood fit is performed using the measured $d E / d x$ distribution and the TOF residual distribution for the tracks found in a cone around the $D^{* \pm}$ candidates [33]. The value of expected $d E / d x$ for a given particle mass hypothesis can be extracted from the universal curve, which depends on the momentum of the particle. In order to perform the likelihood fit, we define a variable $Z(d E / d x)$ for each particle track:

$$
Z(d E / d x)=\log \left(\frac{d E / d x_{m}}{d E / d x_{p}}\right)
$$

where $d E / d x_{m}$ is the measured $d E / d x$ value and $d E / d x_{p}$ represents the expected $\langle d E / d x\rangle$ extracted from the universal curve for the assumed particle mass hypothesis. We use the $Z(d E / d x)$ distribution instead of the measured $d E / d x$ distribution in the likelihood fit because the $Z(d E / d x)$ has a Gaussian distribution to a good approximation.

The probability density functions used to describe the shape of the various components in the likelihood fit are:

- $d E / d x$ universal curve: parameterization in Equation 5.7. The values of the parameters are obtained from the likelihood fit.

- $Z(d E / d x)$ distribution: described using a single Gaussian function:

$$
G(Z)=\frac{e^{-Z^{2} / 2 \sigma_{Z}^{2}}}{\sqrt{2 \pi} \sigma_{Z}}
$$

The resolution $\sigma_{Z}$ is described by the following empirical function:

$$
\sigma_{Z}=\left[a_{z}+b_{z}\left(\left\langle\frac{d E}{d x}\right\rangle-c_{z}\right)\right]\left(\frac{N_{h i t}}{\bar{N}_{h i t}}\right)^{\beta_{z}}
$$


where $N_{h i t}$ is the number of $d E / d x$ hits for the track under consideration, $\bar{N}_{h i t}$ is the mean number of $d E / d x$ hits in the sample of tracks and $\langle d E / d x\rangle$ is extracted from the universal curve (Equation 5.7). The values of parameters $a_{z}, b_{z}, c_{z}, \beta_{z}$ and $\bar{N}_{h i t}$ in Equation 5.10 are also obtained from the combined likelihood fit.

- TOF residual $\left(t_{r}\right)$ distribution: parameterization described in Section 5.1.

The combined $\left(Z(d E / d x), t_{r}\right)$ likelihood function is formulated as:

$$
\mathcal{L}=\sum_{i=\pi, K, p, e} f_{i}\left(p_{T}\right) G(Z) F\left(t_{r}\right)
$$

where $f_{i}\left(p_{T}\right)$ denotes the $p_{T}$ dependent fraction of particle type $i$ in the sample. The notations $F\left(t_{r}\right)$ and $G(Z)$ represent the probability density functions used to describe the shape of the TOF residual $\left(t_{r}\right)$ and the $Z(d E / d x)$ distributions respectively. The likelihood fit is performed in momentum range 0.4 to $30 \mathrm{GeV} / \mathrm{c}$, separately for negatively and positively charged tracks since their $d E / d x$ distributions can be slightly different. This is because positive and negative tracks have opposite curvature in the magnetic field, which can lead to differences in the charge collected at the sense wires. Figure 5.3 shows the projection of the $d E / d x$ universal curve parameterization obtained from the fit, superimposed over the measured $d E / d x$ distribution in data. An example plot of the $Z(d E / d x)$ distribution and the corresponding projections obtained from the likelihood fit is shown in Figure 5.4. The projections indicate that the parameterizations describe the shape of the universal curve and $Z(d E / d x)$ distribution well.

In the next section, we describe a method that is used at CDF to measure the ability of the particle identification techniques to separate different particle types. We also conduct studies to test whether the combined TOF and $d E / d x$ techniques introduce any biases in identifying various particle, which will be discussed in Section 5.4. 


\subsection{Separation power of the particle identification techniques}

After calibrating the TOF and $d E / d x$ response of the detector, it is important to quantify the ability of the particle identification techniques to distinguish between different particle types. The $d E / d x$ separation power between particles of type $i$ and $j$ is defined as:

$$
S(Z)=\left(Z_{i}-Z_{j}\right) / \sigma_{z}
$$

where $S(Z)$ denotes the $d E / d x$ separation power, $Z_{i}$ is denotes $Z(d E / d x)$ calculated using the predicted $d E / d x$, obtained from the universal curve for particle type $i$, and $\sigma_{Z}$ is the width of the $Z(d E / d x)$ distribution.

Similarly, the TOF separation power between particles of type $i$ and $j$ is defined as:

$$
S(T)=\left(T_{i}-T_{j}\right) / \sigma_{t}
$$

where $S(T)$ denotes the TOF separation power, $T_{i}$, is the predicted time of flight for particle type $i$ and $\sigma_{t}$ is the width of the TOF residual distribution.

The TOF system provides very good kaon/pion separation for momenta less than $p<2.0 \mathrm{GeV} / \mathrm{c}$ as shown in Figure 5.5, while for momentum $p<1.4 \mathrm{GeV} / \mathrm{c}$, TOF provides more than $2 \sigma \mathrm{kaon} /$ pion separation. At higher momentum, $d E / d x$ measurement in the tracker provides better separation power, complementing the TOF system. Figure 5.6 shows the $d E / d x$ separation power, separately for negatively and positively charged tracks.

By combining the two particle identification techniques, we obtain at least $1 \sigma$ separation between kaons and pions in the momentum range $0.4<p<6.0 \mathrm{GeV} / \mathrm{c}$. The kaon/proton separation power falls to about $0.5 \sigma$ for $p>2.0 \mathrm{GeV} / \mathrm{c}$. In general, the separation between kaons and pions/protons decreases for $p>2.0 \mathrm{GeV} / \mathrm{c}$. Pions and protons are well separated using the two techniques in the momentum range $0.4<p<6.0 \mathrm{GeV} / \mathrm{c}$. 
In order to study whether the combined particle identification techniques induce any bias in identifying the various particle types, we conduct test studies using samples of pure kaons and pure pions as discussed in the next section. 

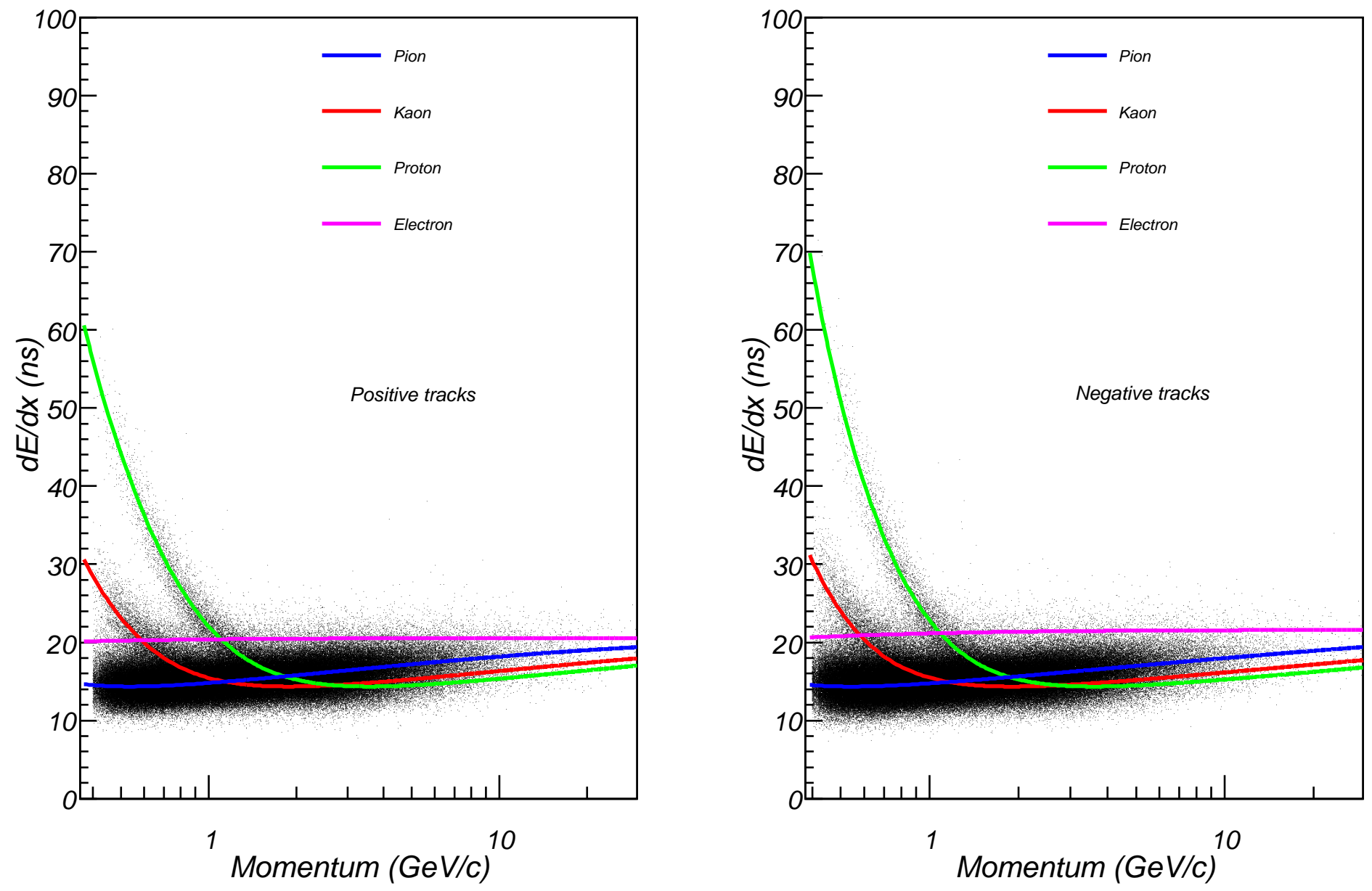

Figure 5.3.: $d E / d x$ distribution in data and projections of the parameterization used to describe the shape of the universal curve. 

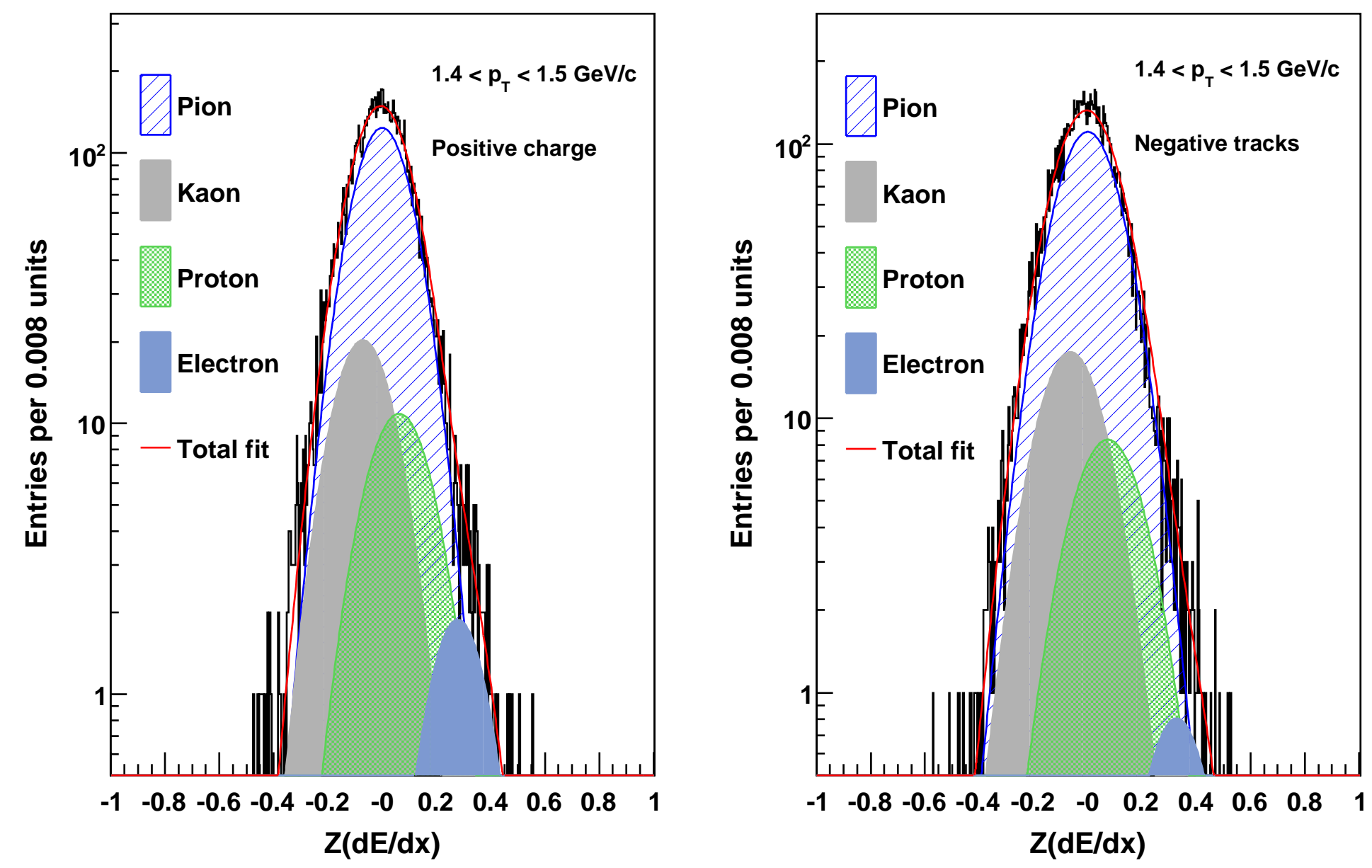

Figure 5.4.: $Z(d E / d x)$ distribution and projections from the likelihood fit, plotted assuming the pion mass hypothesis. 


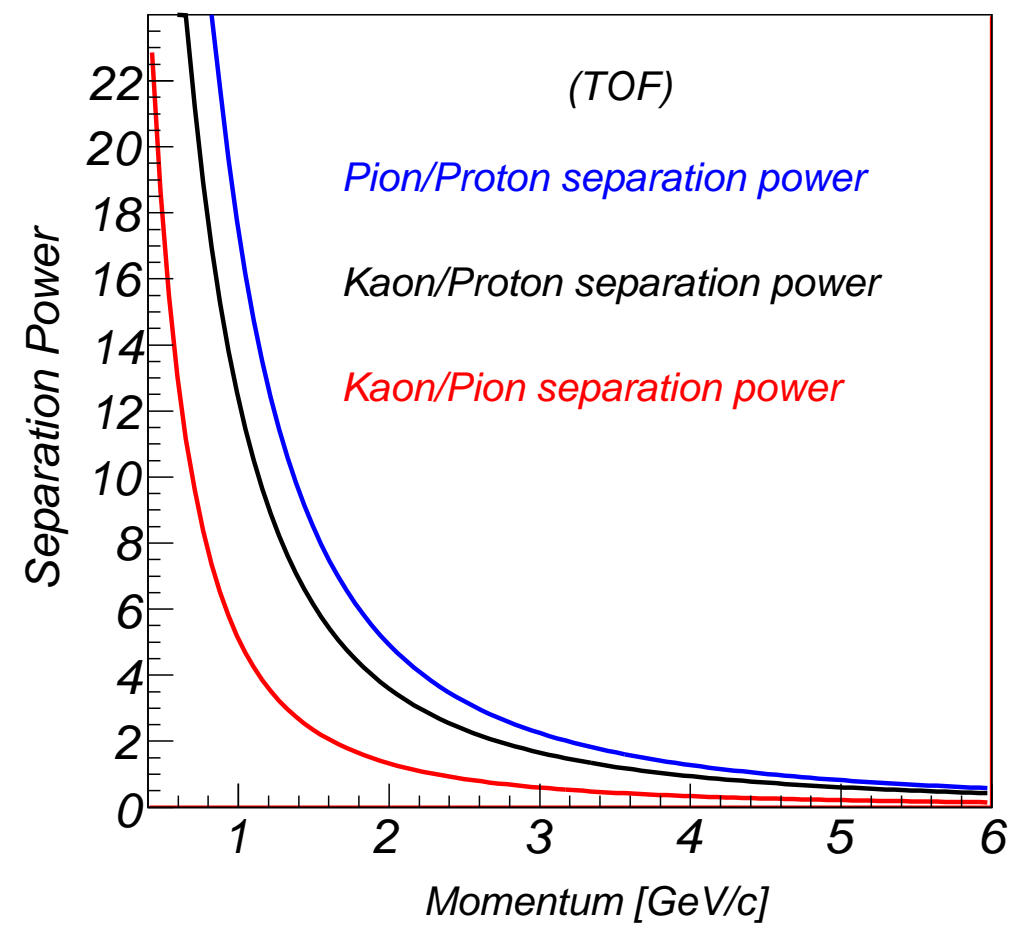

Figure 5.5.: Measured separation power of the particle identification technique using Time-of-Flight. 

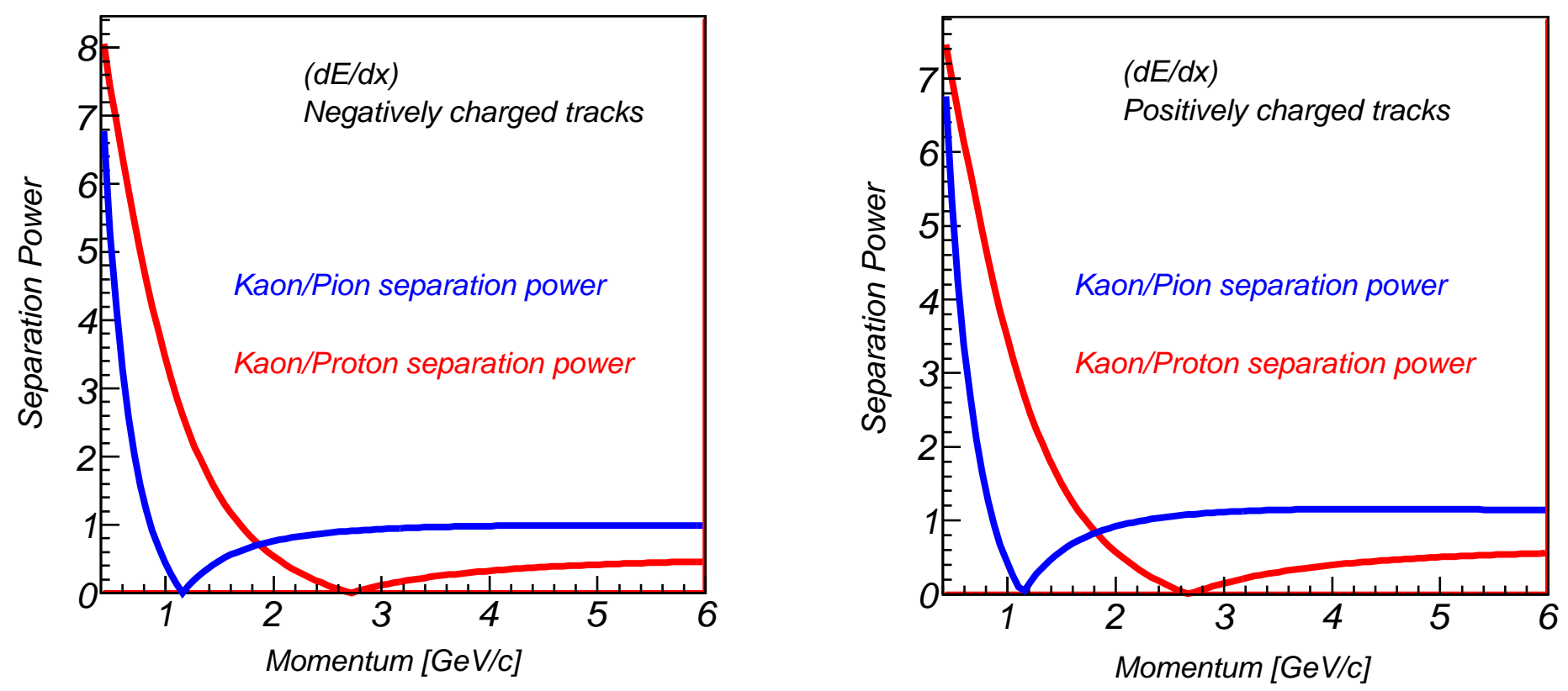

Figure 5.6.: Measured separation power of the particle identification using $d E / d x$ for positively and negatively charged tracks. 


\subsection{Testing the combined particle identification techniques}

Previous sections in this chapter provided a description of the procedure used to calibrate the response of the TOF sub-detector and the $d E / d x$ measurement in the COT. As the next step, we study the ability of the combined TOF and $d E / d x$ particle identification techniques to identify various particle types, by applying them to a sample of pure pions $(\pi)$ from $D^{* \pm} \rightarrow D^{0} \pi^{ \pm}$decays and a sample of pure kaons from $\phi \rightarrow K^{+} K^{-}$decays.

\subsubsection{Study using a pure sample of pions}

In the $D^{* \pm} \rightarrow D^{0} \pi^{ \pm}$sample, the signal component is made of $100 \%$ pions, whereas the background component is considered to be a mixture of kaons, pions and protons. Using the combined particle identification techniques we measure the particle fractions in the signal and background regions. The fractions are measured by performing a combined unbinned likelihood fit, in which the $M\left(D^{* \pm}\right)-M\left(D^{0}\right)$ invariant mass distribution is used to separate the signal and background components. The TOF residual and $Z(d E / d x)$ distributions of the pion track are used to statistically separate the kaon, pion and proton components.

The measured values of the particle fractions in the signal region in bins of $p_{T}(\pi)$ are listed in Table 5.1. The results of the study show that the measured value of the pion fraction in signal region is consistent with the expected value of $100 \%$.

However, it is plausible that the pion fraction in the signal region is measured to be nearly $100 \%$ by construction since the parametrization of the TOF residual distribution was tuned using the same pion sample used in this study. Hence, we test the particle identification techniques using an independent sample of pure kaons. 
Table 5.1: Particle fractions measured in the signal region of the pion sample from $D^{* \pm} \rightarrow D^{0} \pi^{ \pm}$decays.

\begin{tabular}{|c|c|c|c|}
\hline $\begin{array}{c}p_{T} \text { range } \\
(\mathrm{GeV} / \mathrm{c})\end{array}$ & $\begin{array}{c}\text { Pion fraction } \\
\text { (signal) }\end{array}$ & $\begin{array}{c}\text { Kaon fraction } \\
\text { (signal) }\end{array}$ & $\begin{array}{c}\text { Proton fraction } \\
\text { (signal) }\end{array}$ \\
\hline $0.4-0.6$ & $0.9996 \pm 0.0001$ & $+0.0001 \pm 0.0001$ & $-0.0003 \pm 0.0001$ \\
$0.6-0.8$ & $1.0000 \pm 0.0000$ & $-0.0005 \pm 0.0001$ & $-0.0005 \pm 0.0001$ \\
$0.8-1.0$ & $0.9998 \pm 0.0002$ & $-0.0002 \pm 0.0002$ & $-0.0005 \pm 0.0002$ \\
$1.0-1.4$ & $1.0066 \pm 0.0018$ & $-0.0060 \pm 0.0018$ & $-0.0006 \pm 0.0004$ \\
$1.4-2.0$ & $0.9999 \pm 0.0003$ & $+0.0008 \pm 0.0022$ & $-0.0007 \pm 0.0021$ \\
$2.0-6.0$ & $1.0448 \pm 0.0399$ & $-0.0359 \pm 0.0427$ & $-0.0090 \pm 0.0091$ \\
\hline
\end{tabular}

\subsubsection{Study using a pure sample of kaons}

In the $\phi \rightarrow K^{+} K^{-}$sample, the signal component is made of pure kaons and background is considered as a mixture of kaons, pions and protons. Similar to the studies using the pion sample, we measure the particle fractions in the signal and background regions using the combined particle identification techniques.

The fractions are measured by performing a combined un-binned likelihood fit using the $M\left(K^{-} K^{+}\right)$invariant mass distribution to separate the signal and background components. The TOF residual and $Z(d E / d x)$ distributions of the $K^{+}$and $K^{-}$tracks that constitute the $\phi$ candidate are used to separate the kaon, pion and proton components. Since the $\phi$ peak is made of two oppositely charged tracks, we perform the likelihood fit separately for the positively and negatively charged tracks in order to test whether the combined techniques induce any charge-dependent bias. 
The measured values of the particle fractions in the signal region are listed in Table 5.2. The results of the study show that the kaon fraction in the signal region is consistent with the expected value of $100 \%$ in almost all cases.

Table 5.2: Particle fractions measured in the signal component of the $\phi \rightarrow K^{+} K^{-}$ sample.

\begin{tabular}{|c|c|c|c|}
\hline $\begin{array}{c}p_{T} \text { range } \\
(\mathrm{GeV} / \mathrm{c})\end{array}$ & $\begin{array}{c}\text { Pion fraction } \\
\text { (signal) }\end{array}$ & $\begin{array}{c}\text { Kaon fraction } \\
\text { (signal) }\end{array}$ & $\begin{array}{c}\text { Proton fraction } \\
\text { (signal) }\end{array}$ \\
\hline \multicolumn{3}{|c|}{ Positive tracks } \\
\hline $0.4-0.6$ & $-0.0001 \pm 0.0964$ & $0.9934 \pm 0.0206$ & $+0.0067 \pm 0.0203$ \\
$0.6-0.8$ & $+0.0008 \pm 0.0035$ & $1.0046 \pm 0.0068$ & $-0.0054 \pm 0.0067$ \\
$0.8-1.0$ & $+0.0005 \pm 0.0002$ & $1.0084 \pm 0.0068$ & $-0.0089 \pm 0.0068$ \\
$1.0-1.4$ & $+0.0003 \pm 0.0003$ & $1.0153 \pm 0.0066$ & $-0.0156 \pm 0.0065$ \\
$1.4-2.0$ & $+0.0001 \pm 0.0006$ & $1.0122 \pm 0.0096$ & $-0.0122 \pm 0.0096$ \\
$2.0-6.0$ & $-0.0010 \pm 0.0203$ & $1.0486 \pm 0.0233$ & $-0.0476 \pm 0.0232$ \\
\hline \multicolumn{3}{|c|}{ Negative tracks } \\
\hline $0.4-0.6$ & $+0.0001 \pm 0.0211$ & $0.9895 \pm 0.0090$ & $+0.0104 \pm 0.0089$ \\
$0.6-0.8$ & $-0.0004 \pm 0.0076$ & $1.0011 \pm 0.0068$ & $-0.0007 \pm 0.0068$ \\
$0.8-1.0$ & $+0.0003 \pm 0.0003$ & $1.0072 \pm 0.0064$ & $-0.0075 \pm 0.0064$ \\
$1.0-1.4$ & $-0.0005 \pm 0.0010$ & $0.9994 \pm 0.0084$ & $+0.0011 \pm 0.0082$ \\
$1.4-2.0$ & $-0.0020 \pm 0.0006$ & $1.0146 \pm 0.0093$ & $-0.0126 \pm 0.0093$ \\
$2.0-6.0$ & $+0.0015 \pm 0.0182$ & $1.0543 \pm 0.0243$ & $-0.0558 \pm 0.0242$ \\
\hline
\end{tabular}




\subsubsection{Summary of particle identification test studies}

The results of the test studies showed that the combined particle identification techniques do not introduce a very significant bias in identifying various particles for $p_{T}<2.0 \mathrm{GeV} / \mathrm{c}$.

Since the results of the test studies indicate the possibility of a bias in some ranges of $p_{T}>2.0 \mathrm{GeV} / \mathrm{c}$, it is important to account for this bias while using the particle identification techniques for measuring particle fractions in any generic sample. The next section describes a model that was developed in order to account for the bias that results from mis-identification of particle types.

\subsection{Model to account for bias due to particle mis-identification}

When we use the particle identification techniques to measure particle fractions in a pure kaon sample, the measured fractions listed in Table 5.2 indicate a small but significant bias in the kaon and proton fractions in the momentum range 2.06.0 GeV/c. Similarly, the measured particle fractions in a pure pion sample listed in Table 5.1 show a bias in the pion and kaon fractions for 2.0-6.0 GeV/c. This bias can be attributed to the poorer separation power between particles in momentum range greater than $2.0 \mathrm{GeV} / \mathrm{c}$. In the pure pion sample, the large pion component biases the measured kaon fraction, whereas the pion/kaon bias in the pure kaon sample is small since there are not many pions in that sample. Similarly, in the pure kaon sample, the large kaon component biases the proton fraction. Hence, in general terms we can state that a bias introduced in the measured fraction of particle type $i$ due to presence of particle type $j$ in a sample is proportional to the fraction of particle type

$j$ in the sample. This observation forms the basis of the model that is described in this section to account for the bias due to mis-identification. 
We hypothesize that the true particle fractions and the measured particle fractions in a given sample are related using the following linear transformation:

$$
\left(\begin{array}{c}
f_{\pi} \\
f_{k} \\
f_{p}
\end{array}\right)=\left(\begin{array}{ccc}
\alpha_{\pi \pi} & \alpha_{k \pi} & \alpha_{p \pi} \\
\alpha_{\pi k} & \alpha_{k k} & \alpha_{p k} \\
\alpha_{\pi p} & \alpha_{k p} & \alpha_{p p}
\end{array}\right)\left(\begin{array}{c}
\hat{f}_{\pi} \\
\hat{f}_{k} \\
\hat{f}_{p}
\end{array}\right)
$$

where $\hat{f}_{i}$ is the true particle fraction of particle type $i$ and and $f_{i}$ is the measured particle fraction. The parameters $\alpha_{i j}$ give the estimate of the bias induced in the measured fraction of particle type $i$ due to the presence of particle type $j$ in the sample. Ideally, if there is no bias induced due to different particle types, the above $\alpha_{i j}$ matrix would be an identity matrix. However, if a bias exists then the off-diagonal terms in the matrix will be non-zero.

We use the two calibration samples to obtain the values of the elements of the bias-matrix, namely the pion sample from $D^{*}$ decays and the $\phi \rightarrow K^{+} K^{-}$sample that were used for testing the particle identification techniques.

\subsubsection{Bias matrix elements obtained using a pion sample from $D^{*}$ decays}

The signal region in the pion sample is made of $100 \%$ pions. Ideally, the measured kaon and proton fractions in the signal region should be zero. Hence, a non-zero value of the measured kaon and proton fractions gives an estimate of the bias induced due to the presence of pions in the sample. The true particle fractions in the signal region are given by:

$$
\begin{gathered}
\hat{f}_{\pi}=1 \\
\hat{f}_{k}=\hat{f}_{p}=0
\end{gathered}
$$

Using the linear transformation, the measured particle fractions in the signal region are given by:

$$
\begin{aligned}
& f_{\pi}=\alpha_{\pi \pi} \times \hat{f}_{\pi}=\alpha_{\pi \pi} \\
& f_{k}=\alpha_{\pi k} \times \hat{f}_{\pi}=\alpha_{\pi k}
\end{aligned}
$$




$$
f_{p}=\alpha_{\pi p} \times \hat{f}_{\pi}=\alpha_{\pi p}
$$

Hence, the measured particle fractions in the signal region can be used to obtain the values of matrix elements $\alpha_{\pi \pi}, \alpha_{\pi k}$ and $\alpha_{\pi p}$. Table 5.1 lists the values of $f_{\pi}, f_{k}$ and $f_{p}$ that basically give the values of the three above listed bias-matrix elements in ranges of $p_{T}$.

\subsubsection{Bias matrix elements obtained using a $\phi \rightarrow K^{+} K^{-}$sample}

In case of the $\phi \rightarrow K^{+} K^{-}$sample, the $\phi$ signal peak is made of $100 \%$ kaons. Ideally, the measured pion and proton fractions in the signal peak should be zero. Hence, a non-zero value of the measured pion and proton fractions gives an estimate of the bias induced due to the presence of kaons in the sample. The true particle fractions in the signal region are given by:

$$
\begin{gathered}
\hat{f}_{k}=1 \\
\hat{f}_{\pi}=\hat{f}_{p}=0
\end{gathered}
$$

Using the linear transformation, the measured particle fractions in the signal region are given by:

$$
\begin{aligned}
& f_{\pi}=\alpha_{k \pi} \times \hat{f}_{k}=\alpha_{k \pi} \\
& f_{k}=\alpha_{k k} \times \hat{f}_{k}=\alpha_{k k} \\
& f_{p}=\alpha_{k p} \times \hat{f}_{k}=\alpha_{k p}
\end{aligned}
$$

Hence, the measured particle fractions in the signal region can be used to obtain the values of matrix elements $\alpha_{k \pi}, \alpha_{k k}$ and $\alpha_{k p}$. Table 5.2 lists the values of $f_{\pi}, f_{k}$ and $f_{p}$ that basically give the values of the three above listed bias-matrix elements in ranges of $p_{T}$, measured separately for positively and negatively charged tracks. 
Obtaining the remaining three bias matrix elements

The remaining three elements: $\alpha_{p \pi}, \alpha_{p k}$ and $\alpha_{p p}$ can be obtained from the other six bias matrix elements using the constraint that the sum of all particle fractions in a given sample should add up to one.

\subsubsection{Correcting for the bias in a generic sample}

In order to correct for the bias in a generic sample, we define the true particle

fractions $\hat{f}_{\pi}, \hat{f}_{k}$ and $\hat{f}_{p}$ as free parameters in the likelihood fitting procedure and then transform the true fractions into measured fractions in the likelihood function using the equations:

$$
\begin{aligned}
& f_{\pi}=\alpha_{\pi \pi} \hat{f}_{\pi}+\alpha_{k \pi} \hat{f}_{k}+\alpha_{p \pi} \hat{f}_{p} \\
& f_{k}=\alpha_{\pi k} \hat{f}_{\pi}+\alpha_{k k} \hat{f}_{k}+\alpha_{p k} \hat{f}_{p} \\
& f_{p}=\alpha_{\pi p} \hat{f}_{\pi}+\alpha_{k p} \hat{f}_{k}+\alpha_{p p} \hat{f}_{p}
\end{aligned}
$$

By applying the transformation, we take into account the bias induced by different particle types in the fraction of a given particle type and we obtain the values of the true particle fractions from the fit. This mis-identification of particle types is treated as a systematic uncertainty in the measured particle fractions.

\subsection{Effect of kaons decaying in flight}

Kaons produced in $p \bar{p}$ collisions can sometimes decay before traversing the CDF tracking system completely via processed such as $K^{+} \rightarrow \pi^{+} \pi^{0}$ or $K^{+} \rightarrow \mu^{+} \nu_{\mu}$. Some of the kaons that decay in flight will either not be reconstructed or will be reconstructed incorrectly. We need to account for the loss of kaons decaying in flight in the measured kaon fraction so that we can extract the generated kaon fraction in the sample. 
Depending on the location where a kaon decays in the CDF tracking system, one of the following scenarios is possible:

- If the kaon decays inside the SVX detector, the kaon trajectory will not be reconstructed. Instead, the decay product of the kaon will traverse the tracker and is more likely to be reconstructed correctly. If the reconstructed decay product is selected, it will most likely contribute to the measured pion fraction instead of the measured kaon fraction.

- If the kaon decays inside the COT, it will most likely not be reconstructed because it may fail the fit for the track helix in the COT or fail to match the TOF/SVX hits for a single helix. However if such a kaon is reconstructed, then it may contribute to either the measured pion or kaon fraction depending on where the kaon decayed in the COT.

- If the kaon decays near the last layer of the COT, just before the TOF system, it is likely to be reconstructed correctly and may contribute to the measured kaon fraction.

The effect of kaons decaying in flight is studied using a Monte Carlo sample of charged kaons found in a cone around reconstructed $B$ decays within detector acceptance [34]. The study involves measuring two effects: (1) the efficiency that a reconstructed track in the detector is associated with a generated kaon and (2) the probability that the reconstructed track contributes to the measured kaon or pion fraction. In order to measure the kaon component that is identified as pions, particle fractions are measured in a sample of kaons that have a reconstructed track associated with them. The results of the measured particle fractions indicate that a small fraction of kaons are identified as pions in the detector. Table 5.3 lists the efficiencies corresponding to the two effects described above in different $p_{T}$ ranges. The table shows that the fraction of kaons decaying in flight that are identified as pions is more significant in the lower $p_{T}$ ranges. 
Table 5.3: Probability that a kaon will be reconstructed track for different $p_{T}$ ranges. The table also lists the probability that a reconstructed kaon contributes to the measured kaon or pion fraction.

\begin{tabular}{|c|c|c|c|}
\hline$p_{T}$ & $\begin{array}{c}K \text { reconstructed }(\%) \\
\text { as a track }(\%)\end{array}$ & $\begin{array}{c}K \text { identified } \\
\text { as a } K(\%) \\
\mathrm{GeV} / \mathrm{c}\end{array}$ & $\begin{array}{c}K \text { identified } \\
\text { as a } \pi(\%) \\
K_{K}\end{array}$ \\
\hline $0.4-0.7$ & $82.90 \pm 1.39$ & $79.0 \pm 1.50$ & $3.90 \pm 0.71$ \\
$0.7-1.0$ & $91.70 \pm 1.21$ & $88.4 \pm 1.40$ & $3.30 \pm 0.78$ \\
$1.0-1.5$ & $99.35 \pm 0.65$ & $95.4 \pm 1.70$ & $3.95 \pm 1.58$ \\
$1.5-2.5$ & $99.58 \pm 0.72$ & $95.6 \pm 2.30$ & $3.98 \pm 2.19$ \\
$2.5-5.0$ & $100.00_{-0.88}^{+0.00}$ & $97.0 \pm 1.50$ & $3.00 \pm 1.50$ \\
\hline
\end{tabular}

The probabilities listed in Table 5.3 are used to account for the effect of kaons decaying in flight in the measured pion/kaon fraction in a generic sample of tracks. We define the fraction of reconstructed kaons that are subsequently identified as pions using:

$$
w=\frac{K_{\pi}}{K_{K}+K_{\pi}}
$$

The value of the fraction $w$ calculated for different $p_{T}$ ranges is listed in Table 5.4. We correct for the loss of kaons decaying in flight, using the following transformation between the measured $\left(f_{i}\right)$ and true $\left(\hat{f}_{i}\right)$ particle fractions in a generic sample:

$$
\begin{gathered}
f_{K}=(1-w) \hat{f_{K}} \\
f_{\pi}=\hat{f_{\pi}}+w \hat{f_{K}}
\end{gathered}
$$


Table 5.4: Fraction of kaons reconstructed as charged tracks that are subsequently identified as pions.

\begin{tabular}{|c|c|}
\hline$p_{T}$ range $(\mathrm{GeV} / \mathrm{c})$ & $w(\%)$ \\
\hline $0.4-0.7$ & $4.70 \pm 0.82$ \\
$0.7-1.0$ & $3.60 \pm 0.82$ \\
$1.0-1.5$ & $3.98 \pm 1.53$ \\
$1.5-2.5$ & $4.00 \pm 2.12$ \\
$2.5-5.0$ & $3.00 \pm 1.46$ \\
\hline
\end{tabular}

In order to apply the correction on a track-by-track basis that depends on the track $p_{T}$, we obtain a $p_{T}$-dependent function $w\left(p_{T}\right)$ by fitting a first order polynomial function to the $w$-distribution:

$$
w\left(p_{T}\right)=a_{0}+a_{1} p_{T}
$$

The linear fit is shown in Figure 5.7 and the value of parameters $a_{0}, a_{1}$ and the correlation coefficient $\rho\left(a_{0}, a_{1}\right)$ obtained from the fit is listed in Table 5.5.

Table 5.5: Results of the linear fit performed on the distribution of fraction $w$ using the function $w\left(p_{T}\right)=a_{0}+a_{1} p_{T}$. The correlation coefficient is also listed in the table.

\begin{tabular}{|c|c|}
\hline Parameter & Value \\
\hline$a_{0}$ & $0.04483 \pm 0.00771$ \\
$a_{1}$ & $-0.00416 \pm 0.00499$ \\
$\rho\left(a_{0}, a_{1}\right)$ & -0.765881 \\
\hline
\end{tabular}

The function $w\left(p_{T}\right)$ is later used to correct for loss of kaons that decayed in flight in order to get the true kaon fraction in any generic sample. The effect of kaons decaying in flight is treated as one of the sources of systematic uncertainty in the measured 


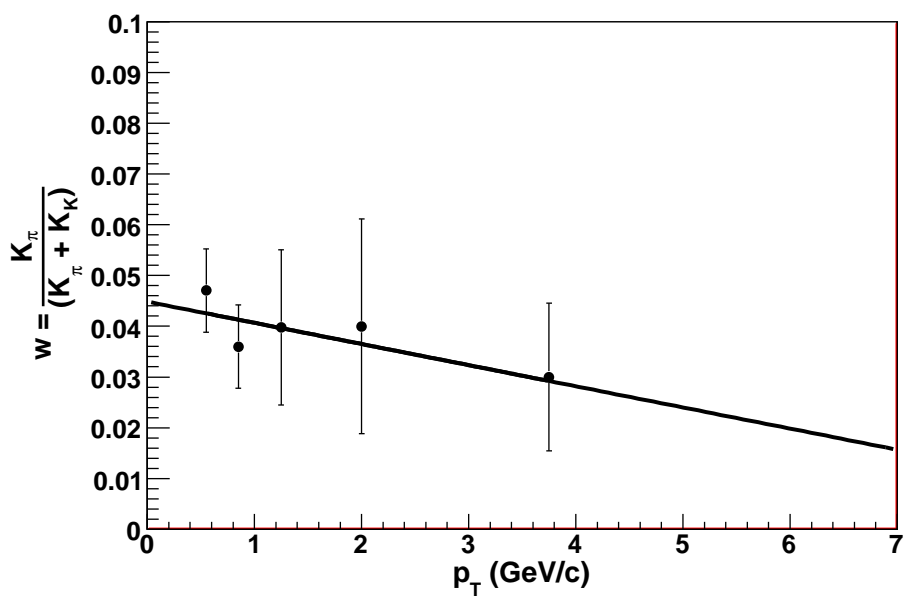

Figure 5.7.: $p_{T}$ distribution of fraction of reconstructed kaons subsequently identified as pions.

kaon fraction in the charm fragmentation analysis present in this dissertation. The procedure for estimating the systematic uncertainty is described in Chapter 7 that provides an overview of the systematic studies conducted in the analysis.

\subsection{Summary}

The particle identification techniques described in this chapter are used to identify kaons in a sample of tracks produced in the vicinity of prompt $D_{s}^{ \pm}$or $D^{ \pm}$mesons in order to study the distribution of kaons produced in the fragmentation of charm quarks. In the next chapter, we present the technique used in the charm fragmentation analysis. 


\section{THE ANALYSIS TECHNIQUE}

The data sample used in the analysis consists of events collected with CDF's two track trigger, described previously in Section 4.2.8, obtained from $360 \mathrm{pb}^{-1}$ of $p \bar{p}$ collisions at $\sqrt{s}=1.96 \mathrm{TeV} / \mathrm{c}^{2}$. Using these events, we reconstruct the $D_{s}^{ \pm} / D^{ \pm} \rightarrow \phi \pi^{ \pm}$, $\phi \rightarrow K^{+} K^{-}$decays and analyze events that have invariant mass in the range $1.75<$ $m(K K \pi)<2.2 \mathrm{GeV} / \mathrm{c}^{2}$. The events collected with the trigger include the prompt $D_{s}^{ \pm} / D^{ \pm}$mesons, which are produced via hadronization of charm quarks, and the secondary $D_{s}^{ \pm} / D^{ \pm}$mesons produced in decays of hadrons containing heavier bottom quarks. In order to extract information pertaining to charm quark fragmentation, we are primarily interested in the prompt $D_{s}^{ \pm} / D^{ \pm}$component. Hence, an important step in the analysis is the separation of the prompt and secondary components, which is discussed in Section 6.1. After separating the prompt and secondary $D_{s}^{ \pm} / D^{ \pm}$components, we apply the particle identification techniques described in Chapter 5 on tracks found near the reconstructed $K K \pi$ candidates to measure the charged particle fractions in the sample. Although, we measure particle fractions around both prompt and secondary $D_{s}^{ \pm} / D^{ \pm}$components, we are primarily interested in measured kaon fraction around the prompt $D_{s}^{ \pm} / D^{ \pm}$component, which gives information about kaon production in association with charm fragmentation. The likelihood fitting procedure used to measure the kaon fraction around both prompt and secondary $D_{s}^{ \pm} / D^{ \pm}$components is described in Section 6.2. The final step in the analysis is to compare the distribution of the measured kaon fraction around the prompt $D_{s}^{ \pm} / D^{ \pm}$component with predictions of the PYTHIA and HERWIG Monte Carlo event generators. 


\subsection{Identification of prompt $D_{s}^{ \pm} / D^{ \pm}$components in the sample}

In order to study kaon production around prompt $D_{s}^{ \pm}$and $D^{ \pm}$mesons, we need to separate these components from the secondary $D_{s}^{ \pm} / D^{ \pm}$and background components in the data sample. We use the invariant mass and impact parameter distributions of the reconstructed $K K \pi$ candidates to identify the various components in the sample as described in this section.

\subsubsection{Separation of $D_{s}^{ \pm} / D^{ \pm}$signal}

The invariant mass distribution of the reconstructed $K K \pi$ candidates with transverse momentum in the range $7<p_{T}<30 \mathrm{GeV} / \mathrm{c}$ is shown in Figure 6.1. As seen in the figure, the $D_{s}^{ \pm}$signal peak (around $1.968 \mathrm{GeV} / \mathrm{c}^{2}$ ) and the $D^{ \pm}$signal peak (around $1.869 \mathrm{GeV} / \mathrm{c}^{2}$ ) are well separated with the background component underneath them. The $D_{s}^{ \pm} / D^{ \pm}$signal components are statistically separated from the background by using the invariant mass distribution in an unbinned likelihood fit. The shape of the signal peaks is described using a sum of two Gaussian functions with the same mean but different widths. The background component is described using a fourth order polynomial function. A broad resonant peak is visible around $2.02 \mathrm{GeV} / \mathrm{c}^{2}$ above the $D_{s}^{ \pm}$peak. The shape of this broad structure is obtained by studying a Monte Carlo sample as described below.

\subsubsection{Broad peak in the $K K \pi$ invariant mass distribution}

In order to find the source of the wide peak, we study a generic sample of $c \bar{c}$ events generated using the PYTHIA event generator. We study a generic sample instead of exclusive decay modes, since we do not have any prior information about the source and need to find all possible decay modes that can contribute to the peak. In case the wide structure has more than one source, we need to account for each contribution in order to determine the correct shape of the peak. 


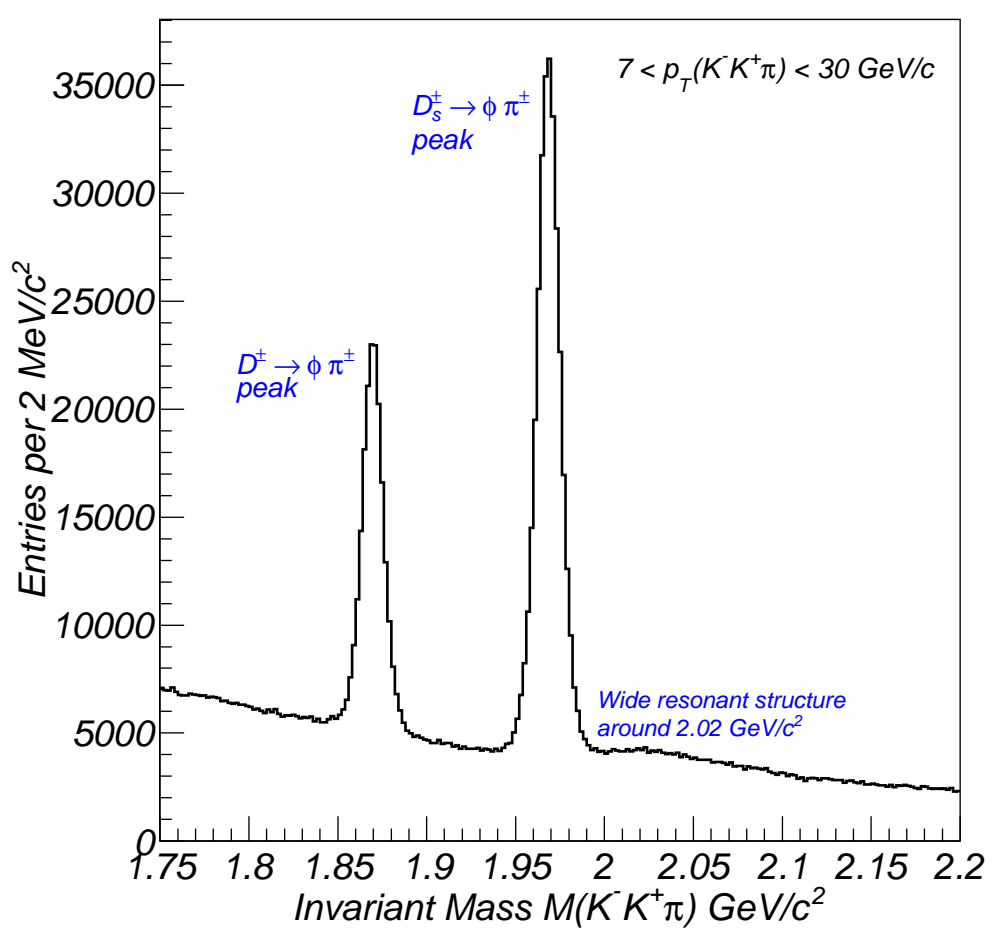

Figure 6.1.: $K K \pi$ invariant mass distribution in data.

Using the momentum of each charged particle generated in the events, we reconstruct three-track combinations, assuming $K K \pi$ particle masses. The $K K \pi$ invariant mass distribution shows the correctly reconstructed $D_{s}^{ \pm} / D^{ \pm}$signal peaks and the wide resonant structure. Then, we check for a common parent particle of the $K K \pi$ combinations that contribute to the resonant structure. We find that only mis-reconstructed $D^{+} \rightarrow K^{-} \pi^{+} \pi^{+}$decays contribute to the resonant structure in the $K K \pi$ invariant mass distribution.

After identifying the source of the bump, we generate Monte Carlo samples of $D^{+} \rightarrow K^{-} \pi^{+} \pi^{+}$decays using the full CDF II detector and trigger simulation, and mis-reconstruct them as $K K \pi$. The resulting $K K \pi$ invariant mass distribution is shown in Figure 6.2. The shape of the resonant peak is obtained by fitting a sum of 
two Gaussian functions with the same mean but different widths, which is also shown in Figure 6.2. This probability density function obtained from Monte Carlo events is used to parameterize the resonant structure in the $K K \pi$ invariant mass distribution in data.

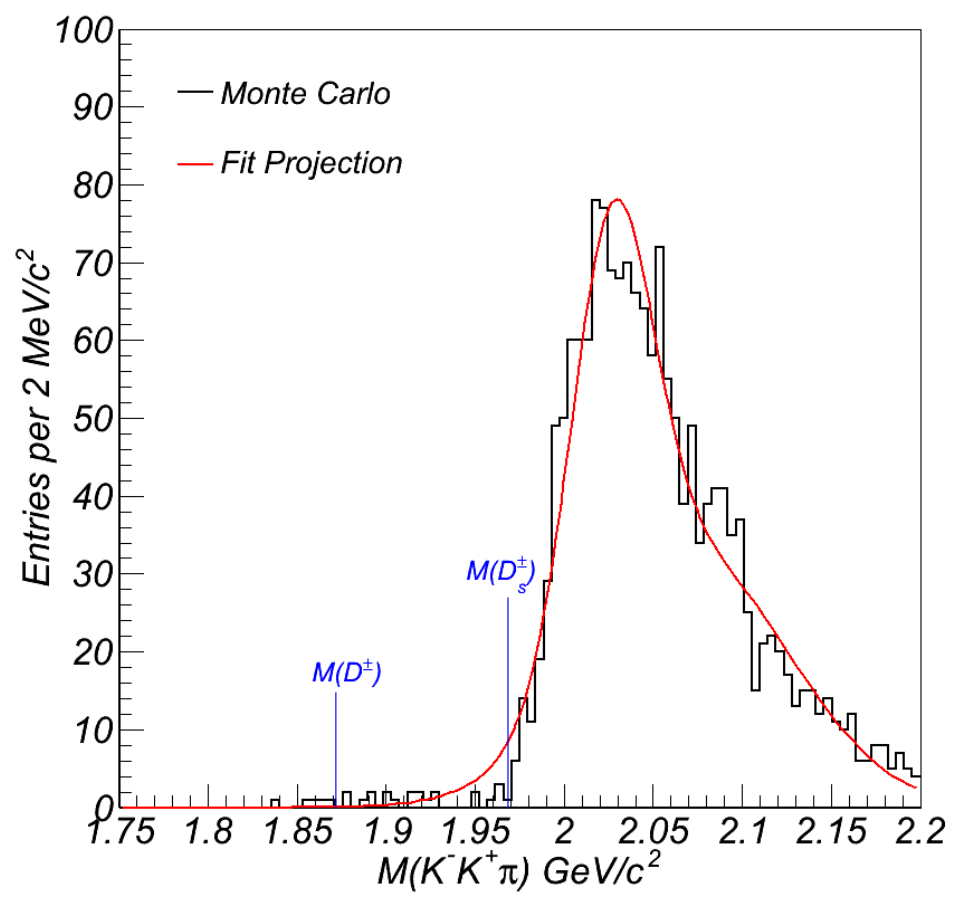

Figure 6.2.: $K K \pi$ invariant mass distribution of mis-reconstructed $D^{+} \rightarrow K^{-} \pi^{-} \pi^{+}$ decays in Monte Carlo events.

\subsubsection{Likelihood fit using the $K K \pi$ invariant mass distribution}

In the fitting procedure, the probability density functions describing the shapes of the various components are used to calculate the likelihood that a given $K K \pi$ candidate is part of $D_{s}^{ \pm}$or $D^{ \pm}$signal, mis-reconstructed $D^{+} \rightarrow K^{-} \pi^{-} \pi^{+}$peak or combinatorial background components. The values of parameters in the probability density functions are obtained from the likelihood fit, which is performed in ranges of 
Table 6.1: Yields of $D_{s}^{ \pm}$and $D^{ \pm}$mesons in ranges of transverse momentum $p_{T}$ of the reconstructed $K K \pi$ candidates.

\begin{tabular}{|c|c|c|}
\hline$p_{T}[\mathrm{GeV} / \mathrm{c}]$ & $D_{s}^{ \pm}$yield & $D^{ \pm}$yield \\
\hline $7-8$ & $56837 \pm 243$ & $30802 \pm 209$ \\
$8-9$ & $53034 \pm 233$ & $28440 \pm 196$ \\
$9-10$ & $42101 \pm 208$ & $22464 \pm 177$ \\
$10-12$ & $52277 \pm 236$ & $26799 \pm 198$ \\
$12-30$ & $59048 \pm 256$ & $27287 \pm 204$ \\
\hline
\end{tabular}

transverse momentum $p_{T}$ of the $K K \pi$ candidates. The yields of $D_{s}^{ \pm} / D^{ \pm}$mesons in the signal peaks, obtained from the fit in ranges of $p_{T}(K K \pi)$ are listed in Table 6.1 . Figure 6.3 shows the projections of the probability density functions for the various components in the $K K \pi$ invariant mass distribution for $7<p_{T}<8 \mathrm{GeV} / \mathrm{c}$. We observe similar level of agreement between data and the projections in the other four $p_{T}(K K \pi)$ ranges in which the likelihood fit is performed.

\subsubsection{Separation of prompt and secondary $D_{s}^{ \pm} / D^{ \pm}$components}

The $D_{s}^{ \pm} / D^{ \pm}$mesons in the signal peaks include both the prompt and secondary components that need to be separated. The technique used for separating the two components is same as the technique that was used in the measurement of the prompt charm meson production cross section $[35,36]$, which basically involves using the impact parameter distribution of the $D_{s}^{ \pm} / D^{ \pm}$mesons with respect to the primary vertex.

As illustrated in Figure 6.4, a $D_{s}^{ \pm} / D^{ \pm}$meson created by the hadronization of a charm quark will be produced at the primary vertex and has zero impact parameter with respect to the primary vertex. However, due to finite resolution of the detector the impact parameter distribution of the prompt component will follow a Gaussian 


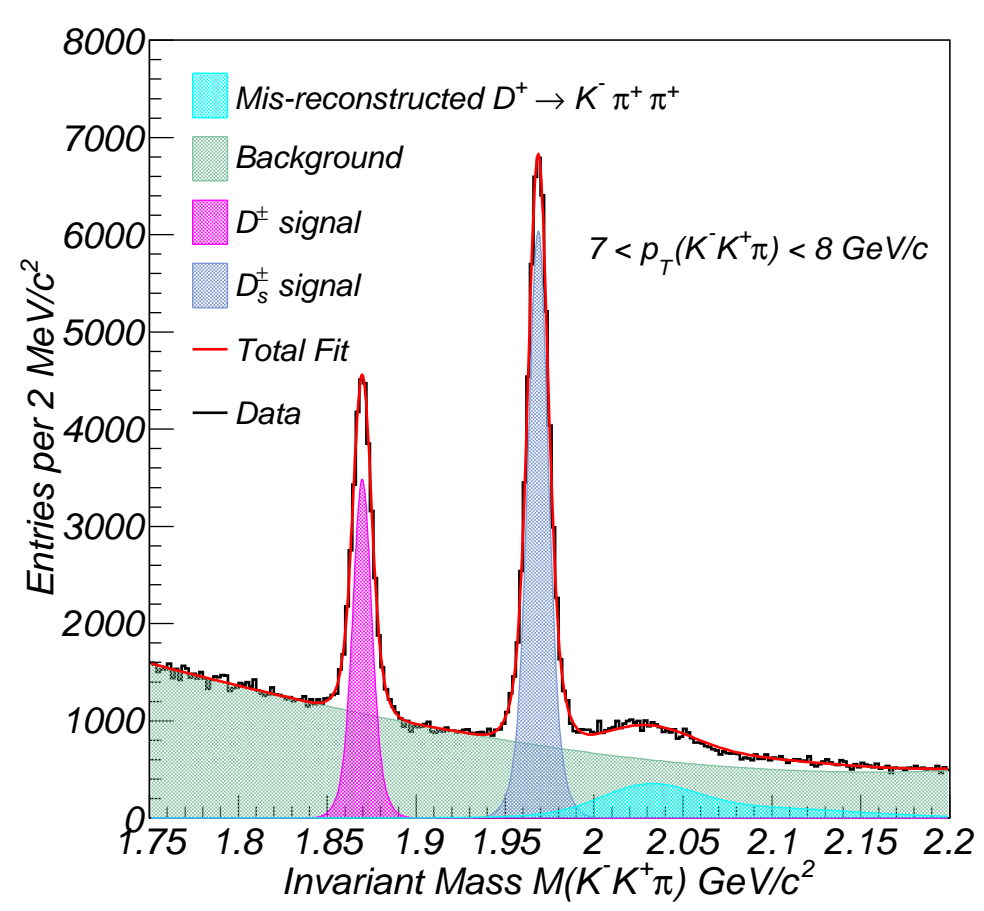

Figure 6.3.: $K K \pi$ invariant mass distribution in data and projections from the likelihood fit for $7<p_{T}<8 \mathrm{GeV} / \mathrm{c}$.

distribution. As shown in Figure 6.4, a secondary $D_{s}^{ \pm} / D^{ \pm}$meson that is produced in the decay of a $\mathrm{B}$ hadron can have non zero impact parameter with respect to the primary vertex. The shape of the secondary component will have a non Gaussian distribution that is smeared by the detector resolution. This difference in the inherent shape of the impact parameter distribution of the two components measured with respect to the primary vertex can be used to separate the prompt and secondary $D_{s}^{ \pm} / D^{ \pm}$components.

\subsubsection{Shape of secondary $D_{s}^{ \pm} / D^{ \pm}$impact parameter distribution}

The shape of the impact parameter distribution of the secondary $D_{s}^{ \pm} / D^{ \pm}$component is obtained by studying Monte Carlo samples of B decays. The rates of secondary 


\section{Prompt $D$ points back to the primary vertex (PV)}

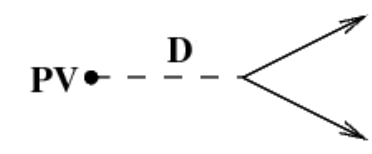

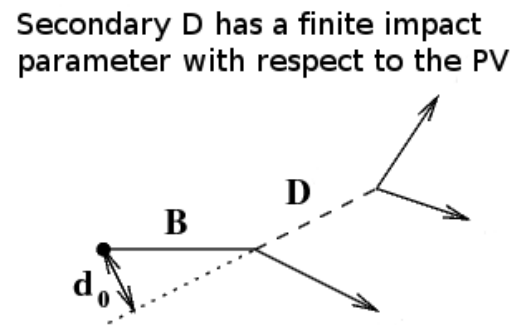

Figure 6.4.: A secondary $D$ meson can have non zero impact parameter $\left(d_{0}\right)$ with respect to the primary vertex, whereas, a prompt $D$ meson will point back to the primary vertex and should ideally have zero impact parameter.

$D_{s}^{ \pm} / D^{ \pm}$production depend on the B branching fractions and the production fractions of various B hadrons. Since not all the B branching fractions have been measured precisely, using an inclusive Monte Carlo sample of B decays to extract the shape of the secondary $D_{s}^{ \pm} / D^{ \pm}$component might not be optimal. This is because the $D_{s}^{ \pm} / D^{ \pm}$ production rates from B decay modes with less precisely measured branching fractions may not be accounted for correctly in the simulation. Instead, we use Monte Carlo samples of exclusive B decay modes and combine the secondary $D_{s}^{ \pm} / D^{ \pm}$impact parameter distribution obtained from each generated B decay that is weighted using:

- the production fraction of the parent B hadron,

- branching fraction of the generated exclusive B decay mode,

- two track trigger and $K K \pi$ candidate reconstruction efficiency.

Monte Carlo samples of exclusive B decays were generated including the full CDF II detector and trigger simulation. The $D_{s}^{ \pm} / D^{ \pm}$mesons that were produced in the generated $\mathrm{B}$ decay modes were forced to decay via the decay channel $D_{s}^{ \pm} / D^{ \pm} \rightarrow$ $\phi \pi^{ \pm}, \phi \rightarrow K^{+} K^{-}$. The reconstructed $K K \pi$ candidates were required to satisfy the two track trigger criteria described in Section 4.2.8. The generated exclusive B decay modes that produce a secondary $D_{s}^{ \pm} / D^{ \pm}$meson and the corresponding efficiency for 
Table 6.2: Yields of exclusive samples of B decays that were generated for studying the impact parameter distributions of secondary $D$ mesons. The table also lists the number of $K^{+} K^{-} \pi$ candidates that satisfy track quality criteria, offline selection cuts and SVT-like pair requirement with $7<p_{T}<30 \mathrm{GeV} / \mathrm{c}$.

\begin{tabular}{|c|c|c|c|}
\hline Decay mode & Generated & $\begin{array}{c}\text { Passed offline and } \\
\text { trigger requirements }\end{array}$ & Efficiency \\
\hline$B_{s}^{0} \rightarrow D_{s}^{-} \pi^{+}$ & 9618329 & 51016 & $0.53 \%$ \\
$B_{s}^{0} \rightarrow D_{s}^{-} \pi^{+} \pi^{-} \pi^{+}$ & 9522146 & 31070 & $0.33 \%$ \\
$B^{0} \rightarrow D^{-} \pi^{+}$ & 9609157 & 64406 & $0.67 \%$ \\
$B^{+} \rightarrow \bar{D}^{0} D_{s}^{+}$ & 4974607 & 21626 & $0.43 \%$ \\
$B^{+} \rightarrow \bar{D}^{0} D^{+}$ & 4995190 & 25036 & $0.50 \%$ \\
$B^{+} \rightarrow D^{-} \pi^{+} \mu^{+} \nu_{\mu}$ & 4971322 & 12889 & $0.26 \%$ \\
\hline
\end{tabular}

finding events that have a $K K \pi$ candidate satisfying the trigger criteria are listed in Table 6.2.

For each exclusive $B$ decay sample listed in Table 6.2 , we obtain the impact parameter distribution for the reconstructed $K K \pi$ candidates. These individual impact parameter distributions are weighted and combined to obtain a distribution that has contributions from various sources, emulating the secondary $K K \pi$ impact parameter distribution in data. Using this procedure, we obtain a combined $K K \pi$ impact parameter distribution, separately for secondary $D_{s}^{ \pm}$and secondary $D^{ \pm}$mesons.

In order to extract the shape of the secondary $D_{s}^{ \pm} / D^{ \pm}$impact parameter component, we perform an unbinned likelihood fit using the combined $K K \pi$ impact parameter distributions. A sum of two exponential functions is used to parameterize the impact parameter distribution in the fit, which is performed in ranges of $p_{T}$ of the $K K \pi$ candidates [37]. The combined $K K \pi$ impact parameter distributions and the projections from the likelihood fit in the lowest $p_{T}$ range are shown in Figure 6.5. 
We obtain similar levels of agreement between the combined $K K \pi$ impact parameter distribution and the projections in the other four $p_{T}$ ranges in which the likelihood fit is performed. 

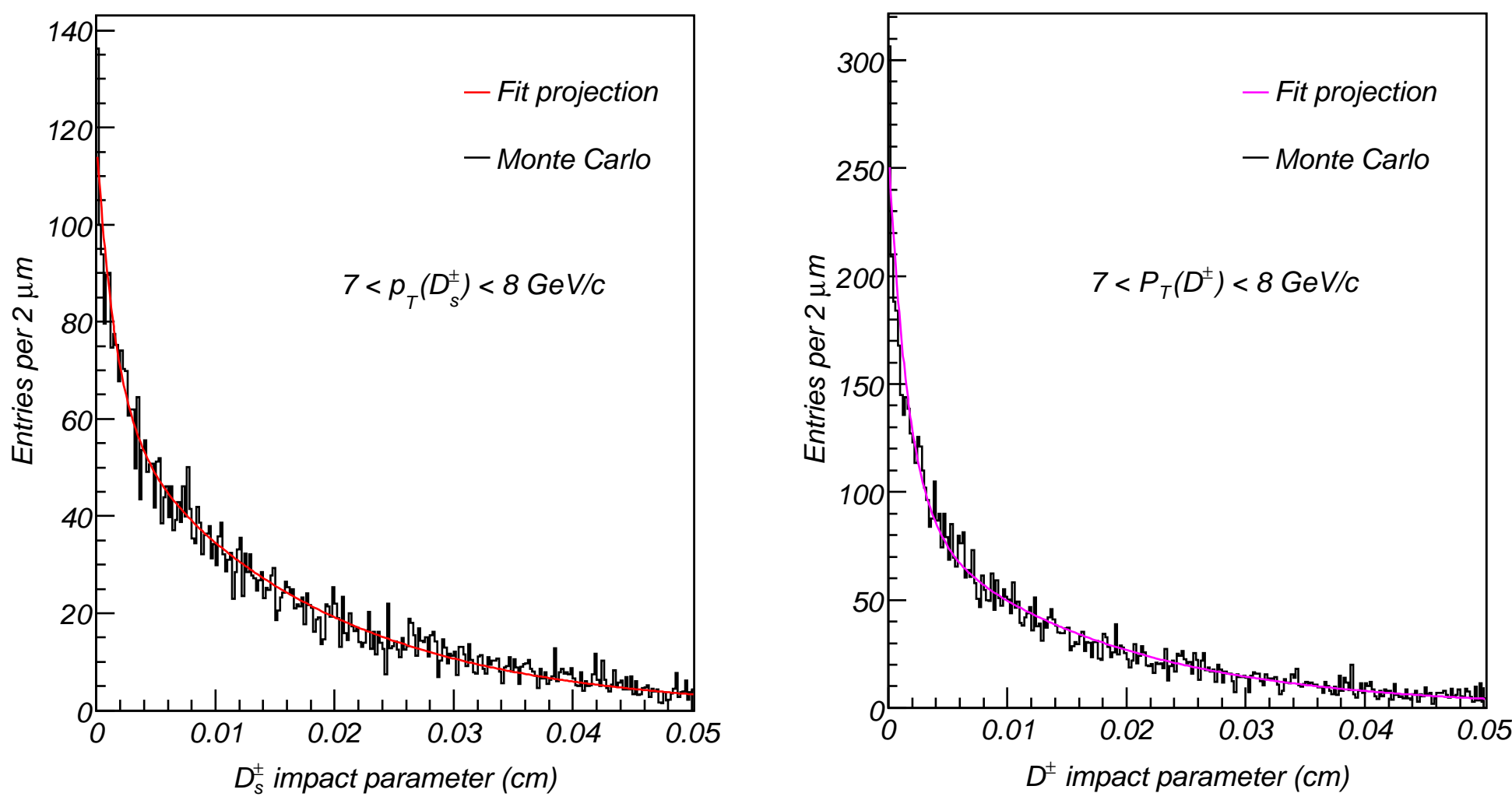

Figure 6.5.: Impact parameter distribution of $K K \pi$ candidates found in Monte Carlo samples of B decays, superimposed with projections obtained from the likelihood fit. 
The probability density function for secondary $D_{s}^{ \pm} / D^{ \pm}$mesons obtained by studying Monte Carlo samples of B decays is convoluted with the prompt resolution Gaussian function, prior to using it to describe the secondary $D_{s}^{ \pm} / D^{ \pm}$component in the $K K \pi$ impact parameter distribution in data. This is done in order to incorporate the detector resolution effects in the probability density functions that are obtained using the generator level information in the Monte Carlo samples.

\subsubsection{Fits to the $K K \pi$ invariant mass and impact parameter distributions}

The separation of prompt and secondary components is done statistically by performing an unbinned likelihood fit using the invariant mass, $M(K K \pi)$, distribution and the impact parameter, $d_{0}(K K \pi)$, distributions of the reconstructed $K K \pi$ candidates. In this likelihood fit, the probability density functions used to describe the shape of the various components in the $M(K K \pi)$ and $d_{0}(K K \pi)$ distributions give the likelihood that a given $K K \pi$ candidate is part of the prompt $D_{s}^{ \pm} / D^{ \pm}$component in the signal peak, the secondary $D_{s}^{ \pm} / D^{ \pm}$component in the signal peak, the wide resonant peak or the combinatorial background component. The following functions are used to describe the shape of the various components in the likelihood fit:

- the shape of the prompt $D_{s}^{ \pm} / D^{ \pm}$component in the $d_{0}(K K \pi)$ distribution is described using a sum of two Gaussian functions, both centered at zero but with different widths.

- the shape of the background component in the $d_{0}(K K \pi)$ distribution is described using the sum of a Gaussian function and an exponential function. We compare the impact parameter distribution in the lower, middle and upper sideband regions in the $M(K K \pi)$ distribution and find that the background impact parameter is independent of mass. Hence, we use the same function to describe the shape of the background impact parameter in the sideband region and under the $D_{s}^{ \pm} / D^{ \pm}$signal peaks. 
- the shape of the secondary $D_{s}^{ \pm} / D^{ \pm}$component in the $d_{0}(K K \pi)$ distribution is described using the probability density function obtained from Monte Carlo samples of B decays described in Section 6.1.5 convoluted with the prompt resolution function.

- the shape of components in the $M(K K \pi)$ distribution is described using the probability density functions discussed in sections 6.1.1 and 6.1.2.

The results of the likelihood fit indicate that approximately $75 \%$ of $D_{s}^{ \pm}$mesons are prompt, whereas approximately $93 \%$ of $D^{ \pm}$mesons contribute to the prompt component in the data. The fraction of the prompt $D_{s}^{ \pm} / D^{ \pm}$mesons obtained from the likelihood fit in various ranges of $p_{T}(K K \pi)$ are listed in Table 6.3.

The lifetime of a $D^{ \pm}$meson $(c \tau=311.8 \mu \mathrm{m})$ is more than twice the lifetime of a $D_{s}^{ \pm}$meson $(c \tau=149.9 \mu \mathrm{m})$. Hence, the trigger efficiency for prompt $D_{s}^{ \pm}$will be lower than the trigger efficiency for prompt $D_{s}^{ \pm}$. Moreover, the production rates of secondary $D_{s}^{ \pm}$and secondary $D^{ \pm}$from the combined $B / B^{0} / B_{s}^{0} /$ b-baryon decays is similar [1]. Therefore, we expect the number of triggered secondary $D_{s}^{ \pm} / D^{ \pm}$mesons to be similar, but fewer prompt $D_{s}^{ \pm}$will be collected by the trigger compared to prompt $D_{s}^{ \pm}$mesons. Hence, the measured prompt $D^{ \pm}$fraction is higher compared to the measured prompt $D_{s}^{ \pm}$fraction as shown in Table 6.3.

The systematic uncertainty in the prompt fraction listed in the table is calculated by propagating the statistical uncertainties in the parameters of the secondary probability density functions that were fitted to the Monte Carlo samples described in Section 6.1.5. The impact parameter projections in the $D_{s}^{ \pm} / D^{ \pm}$signal region defined within $\pm 3 \sigma$ from the $D_{s}^{ \pm} / D^{ \pm}$signal peak are shown in Figure 6.6 for the lowest $p_{T}$ range. The projections show that we obtain good statistical separation between the prompt and secondary components in the $D_{s}^{ \pm} / D^{ \pm}$signals.

As described in this section, we used the $K K \pi$ invariant mass and impact parameter distributions to statistically separate the prompt $D_{s}^{ \pm} / D^{ \pm}$components from the secondary and background components in the data. The next step in the analysis 
Table 6.3: Values for prompt $D_{s}^{ \pm}$and prompt $D^{ \pm}$fraction in the data sample in ranges of transverse momentum $p_{T}$ of the reconstructed $K K \pi$ candidates. The systematic uncertainty in the prompt fraction listed in the table is calculated by propagating the statistical uncertainties in the secondary templates that are obtained using Monte Carlo samples of exclusive B decays.

\begin{tabular}{|c|c|c|}
\hline$p_{T}$ range & $\begin{array}{c}\text { Prompt } D_{s}^{ \pm} \\
\text {fraction } \\
\mathrm{GeV} / \mathrm{c}\end{array}$ & $\begin{array}{c}\text { Prompt } D^{ \pm} \\
\text {fraction }\end{array}$ \\
\hline $7-8$ & $0.7347 \pm 0.0042 \pm 0.0020$ & $0.9293 \pm 0.0061 \pm 0.0010$ \\
$8-9$ & $0.7547 \pm 0.0073 \pm 0.0025$ & $0.9433 \pm 0.0095 \pm 0.0019$ \\
$9-10$ & $0.7477 \pm 0.0072 \pm 0.0028$ & $0.9283 \pm 0.0093 \pm 0.0017$ \\
$10-12$ & $0.7574 \pm 0.0088 \pm 0.0010$ & $0.9640 \pm 0.0110 \pm 0.0030$ \\
$12-30$ & $0.7481 \pm 0.0096 \pm 0.0037$ & $0.9644 \pm 0.0130 \pm 0.0036$ \\
\hline
\end{tabular}


is to apply the particle identification techniques described in Chapter 5, in order to measure the fractions of kaons, pions and protons in a sample of charged particles produced in association with $D_{s}^{ \pm} / D^{ \pm}$mesons. 

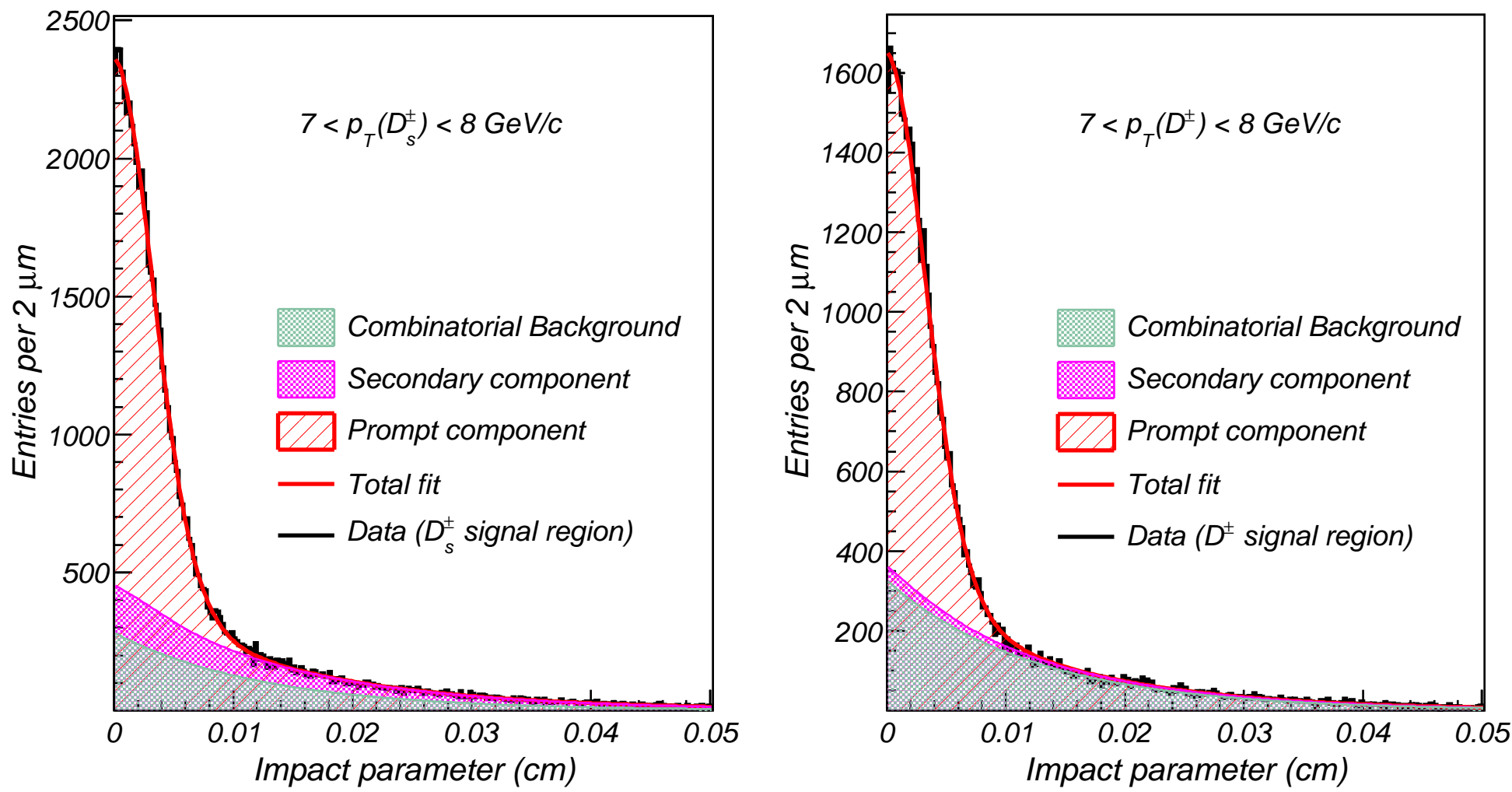

Figure 6.6.: Impact parameter distribution of the $K K \pi$ candidates in the $D_{s}^{ \pm} / D^{ \pm}$signal region and projections from the likelihood fit showing the prompt, secondary and background components. 


\subsection{Measurement of the kaon fraction around prompt $D_{s}^{ \pm} / D^{ \pm}$mesons}

We are primarily interested in measuring the kaon fraction around the prompt $D_{s}^{ \pm}$and prompt $D^{ \pm}$components for studying kaon production in association with the creation of $D_{s}^{ \pm} / D^{ \pm}$mesons via charm quark fragmentation. For the fragmentation study, we select the maximum- $p_{T}$ track in a cone of radius $\Delta R=0.7$ around a $K K \pi$ candidate based on the hypothesis that the maximum- $p_{T}$ is more likely to be correlated with the production of a heavy meson in the fragmentation process. The radius of the cone, $\Delta R$ is defined as:

$$
\Delta R=\sqrt{\Delta \phi^{2}+\Delta \eta^{2}}
$$

where $\Delta \phi$ is the azimuthal angle between the track and the $D$ candidate and $\Delta \eta$ is the difference in their pseudo-rapidity.

For about $77 \%$ of the reconstructed $K K \pi$ candidates, we find only one track per cone that satisfys the selection criteria. For only the remaining $23 \%$ of the reconstructed $K K \pi$ candidates, we pick the maximum- $p_{T}$ track out of two or three tracks found in the cone. In addition, by studying simulated $c \bar{c}$ events generated using PYTHIA, we find that the kinematic properties of the maximum- $p_{T}$ track in the cone are not affected by the underlying event activity as shown in an example plot in Figure 6.7.

\subsubsection{Likelihood fitting procedure for measuring charged particle fractions}

In order to measure the kaon fractions around prompt $D_{s}^{ \pm}$and $D^{ \pm}$, we perform a multidimensional unbinned likelihood fit using the following distributions in data:

1. $K K \pi$ invariant mass distribution: for separating $D_{s}^{ \pm} / D^{ \pm}$signal, wide resonant peak and combinatorial background components.

2. Impact parameter $d_{0}$ distribution for the reconstructed $K K \pi$ candidates: for separating prompt and secondary $D_{s}^{ \pm} / D^{ \pm}$components in the signal peak. 


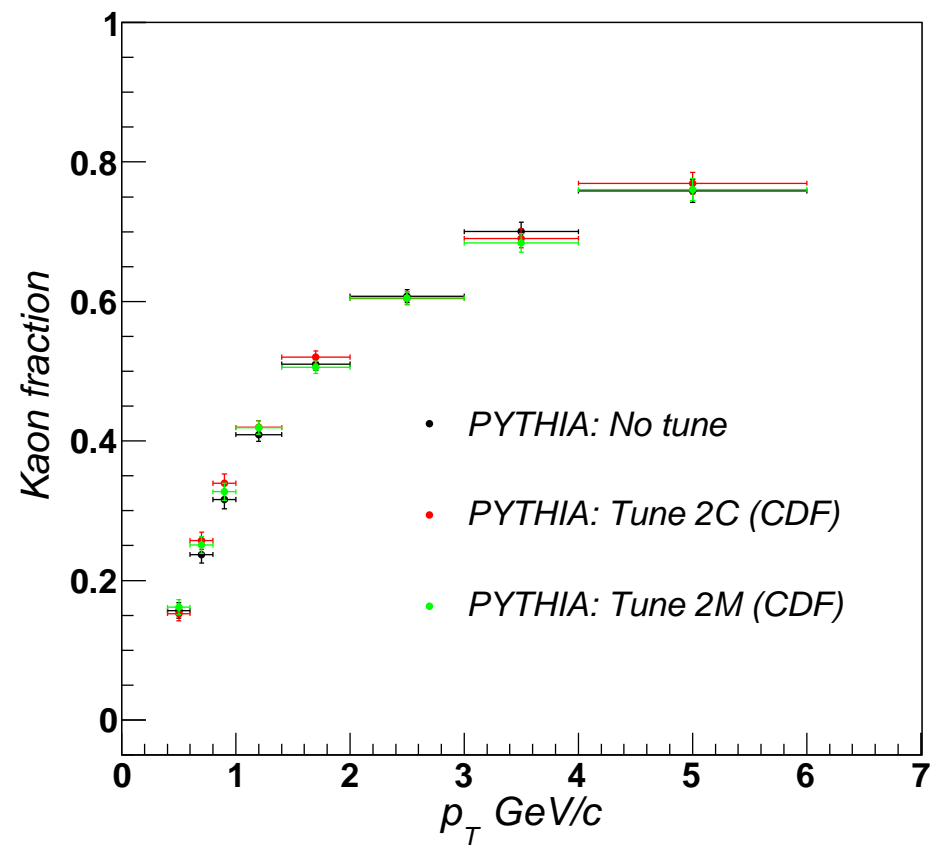

Figure 6.7.: The fraction of kaons in the sample of maximum- $p_{T}$ tracks found in a cone around $D_{s}^{ \pm}$mesons in events generated using PYTHIA. The $p_{T}$ distribution is plotted for PYTHIA samples generated by including and excluding the tuning of the underlying event in the simulation. As we can see in the plot, the tuning does not affect the $p_{T}$ distribution of the maximum- $p_{T}$ tracks found in the cone. The two tunes use different sets of parameters to describe the underlying event.

3. TOF residual distribution of the maximum- $p_{T}$ track: for identifying kaons, pions and protons; with better separation power in lower momentum regime.

4. $Z(d E / d x)$ distribution of the maximum- $p_{T}$ track: for identifying kaons, pions and protons in medium momentum regime, complementing the TOF system.

The underlying principle of the multidimensional fitting procedure is as follows: given the invariant mass and impact parameter of a $K K \pi$ candidate, we can use the probability density functions describing the shape of the various components in the $K K \pi$ invariant mass and impact parameter distributions to calculate the likelihood that the 
particular $K K \pi$ candidate is a prompt $D_{s}^{ \pm} / D^{ \pm}$meson, a secondary $D_{s}^{ \pm} / D^{ \pm}$meson or part of the background component. Using the probability density functions for the TOF and $d E / d x$ distributions, we can calculate the likelihood that the maximum- $p_{T}$ track found in the cone around a reconstructed $K K \pi$ candidate is a kaon, pion or proton.

In the fit, the likelihood per event is formulated as follows:

$$
\begin{aligned}
\mathcal{L}\left(m, d_{0}, t_{r}, z\right)=\left(1-f_{D_{s}^{ \pm}}-f_{D^{ \pm}}\right) F_{B}\left(m, d_{0}, t_{r}, z\right) & +f_{D_{s}^{ \pm}} F_{D_{s}^{ \pm}}\left(m, d_{0}, t_{r}, z\right) \\
& +f_{D^{ \pm}} F_{D^{ \pm}}\left(m, d_{0}, t_{r}, z\right)
\end{aligned}
$$

where $f_{D_{s}^{ \pm}}$and $f_{D^{ \pm}}$is the fraction of all reconstructed $K K \pi$ candidates attributed to the $D_{s}^{ \pm}$and $D^{ \pm}$signals respectively. The variables $m$ and $d_{0}$ represent the $K K \pi$ invariant mass and $K K \pi$ candidate impact parameter. The TOF residual and $Z(d E / d x)$ of the maximum- $p_{T}$ track found in the cone are denoted by $t_{r}$ and $z$ respectively. The notations $F_{D_{s}^{ \pm}}\left(m, d_{0}, t_{r}, z\right)$ and $F_{D^{ \pm}}\left(m, d_{0}, t_{r}, z\right)$ denote the multidimensional probability density functions for the invariant mass, impact parameter and particle identification measurements. The corresponding multi-dimensional probability density function for the background component is denoted by $F_{B}\left(m, d_{0}, t_{r}, z\right)$. The expression for the multi-dimensional probability density function for $D_{s}^{ \pm}$or $D^{ \pm}$ is given by:

$$
F_{D_{i}}\left(m, d_{0}, t_{r}, z\right)=M_{D_{i}}(m)\left[p_{D_{i}} P_{D_{i}}\left(d_{0}, t_{r}, z\right)+\left(1-p_{D_{i}}\right) S_{D_{i}}\left(d_{0}, t_{r}, z\right)\right]
$$

where $M_{D_{i}}(m)$ is the probability density function that describes the shape of the $D_{s}^{ \pm}$or $\left.D^{ \pm}\right\}$signals in the $K K \pi$ invariant mass distributions and $p_{D_{i}}$ is the prompt fraction for $D_{s}^{ \pm}$or $D^{ \pm}$mesons.

$$
P_{D_{i}}\left(d_{0}, t_{r}, z\right)=F_{D_{i}}^{p}\left(d_{0}\right) \sum_{j=\pi, K, p} f_{j}^{p \mid i} T_{j}\left(t_{r}\right) G_{j}(z)
$$

where $F_{D_{i}}^{p}\left(d_{0}\right)$ describes the shape of the prompt component in the impact parameter distribution and $f_{j}^{p \mid i}$ represent the fraction of particle type $j \in\{\pi, K, p\}$ measured around the prompt $D_{i}$ component, for $D_{i} \in\left\{D_{s}^{ \pm}, D^{ \pm}\right\}$. 


$$
S_{D_{i}}\left(d_{0}, t_{r}, z\right)=F_{D_{i}}^{s}\left(d_{0}\right) \sum_{j=\pi, K, p} f_{j}^{s \mid i} T_{j}\left(t_{r}\right) G_{j}(z)
$$

where $F_{D_{i}}^{s}\left(d_{0}\right)$ describes the shape of the secondary component in the impact parameter distribution and $f_{j}^{s \mid i}$ represent the fraction of particle type $j \in\{\pi, K, p\}$ measured around the secondary $D_{i}$ component. In equations 6.3 and $6.4, T_{j}\left(t_{r}\right)$ and $G_{j}(z)$ represent the probability density functions for the TOF residual distribution and the $Z(d E / d x)$ distribution respectively. In Equation 6.1, the multi-dimensional probability density function for the background component is given by:

$$
F_{B}\left(m, d_{0}, t_{r}, z\right)=M_{B}(m) F_{B}\left(d_{0}\right) \sum_{j=\pi, K, p} f_{j}^{B} T_{j}\left(t_{r}\right) G_{j}(z)
$$

where $M_{B}(m)$ and $F_{B}\left(d_{0}\right)$ describe the shape of the background component in the $K K \pi$ invariant mass and impact parameter distribution respectively. The notation $f_{j}^{B}$ represents the fraction of particle type $j \in\{\pi, K, p\}$ measured in the background component.

Although we measure particle fractions around the all components separately in the fitting procedure, we are mainly interested in the kaon fraction around the prompt $D_{s}^{ \pm} / D^{ \pm}$component for a direct comparison with predictions of the fragmentation models used in the Monte Carlo event generators.

\subsubsection{Kaon fraction extracted from the likelihood fitting procedure}

The kaon fraction measured around prompt $D_{s}^{ \pm} / D^{ \pm}$mesons in ranges of transverse momentum $\left(p_{T}\right)$ of the maximum- $p_{T}$ track found in the cone is shown in Figure 6.8. The kaon fraction is measured in two charge scenarios:

1. opposite sign charge scenario, where the track in the cone and the reconstructed $K K \pi$ candidate have opposite charge.

2. same sign charge scenario, where the track in the cone and the reconstructed $K K \pi$ candidate have same charge. 
In addition to measuring the kaon fraction as a function of $p_{T}$, we also measure the kaon fraction in ranges of other kinematic variables. A detailed description and discussion of the results for all the kinematic variables is provided in Chapter 8.

The measured kaon fraction shown in Figure 6.8 has statistical uncertainty that is obtained from the likelihood fitting procedure. The distributions in the figure show fluctuations that do not seem to be consistent within the bounds of the statistical uncertainties that are generally small. Hence, it is important to study possible systematic sources that could lead to these fluctuations in the distributions. In the next chapter, we describe the evaluation of systematic uncertainties in the measured kaon fraction. 

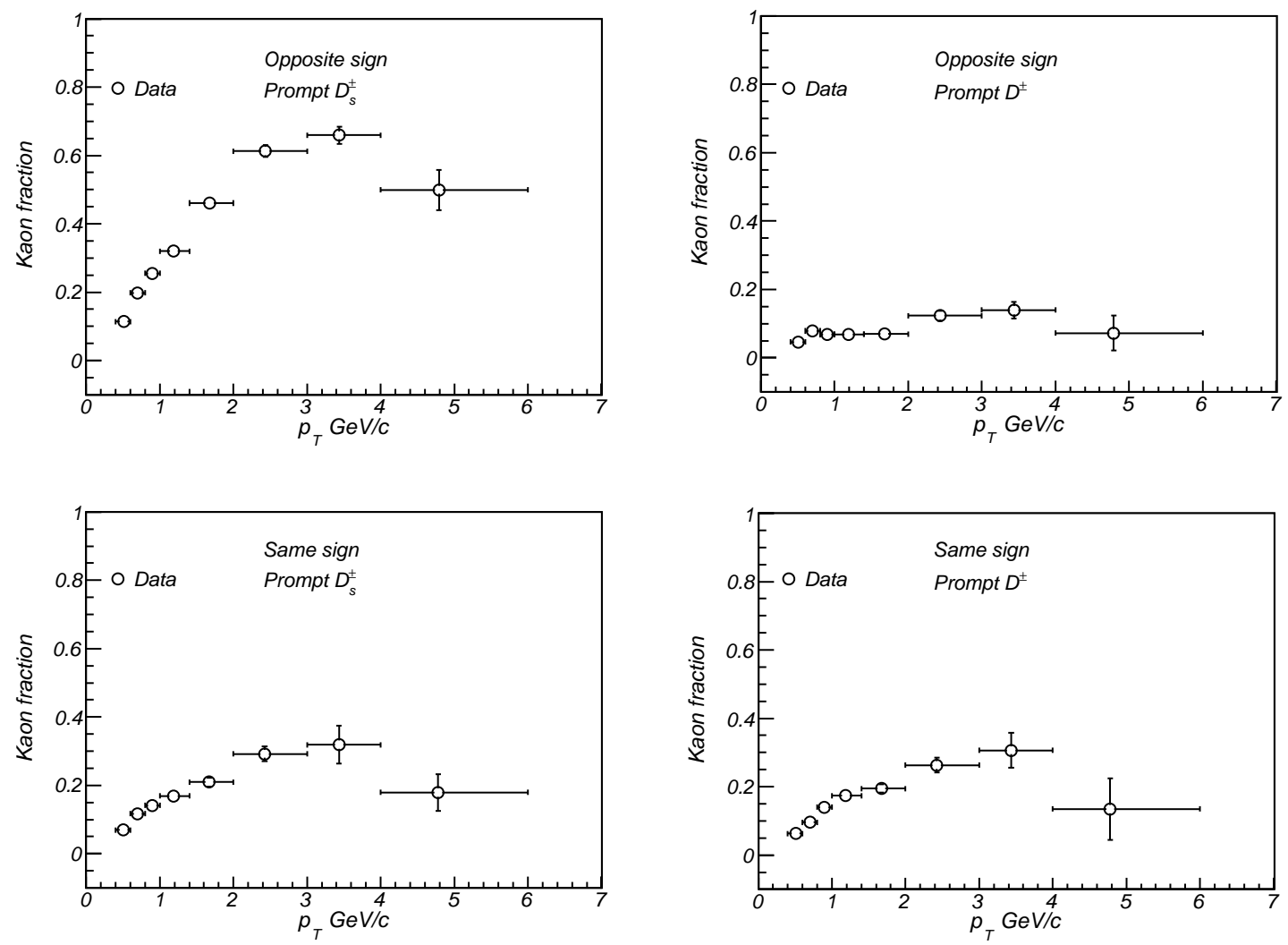

Figure 6.8.: Distribution of kaon fraction measured around prompt $D_{s}^{ \pm} / D^{ \pm}$mesons in ranges of transverse momentum $\left(p_{T}\right)$ of the maximum- $p_{T}$ track found in the cone. The error bars depict only statistical uncertainty obtained from the likelihood fit. 


\section{OVERVIEW OF SYSTEMATIC STUDIES}

Before comparing the results described in the previous chapter with the fragmentation models, we must evaluate the size of any systematic effects that could introduce biases. This chapter describes several potential sources of bias and the procedures used to quantify how large an effect they could have on the measured kaon fractions.

\subsection{Systematic uncertainty due to effect of kaons decaying in flight}

As described in Section 5.6, kaons produced in $p \bar{p}$ collisions can sometimes decay before traversing the CDF tracking systems and can be instead identified as pions. We correct for the loss of kaons decaying in flight to obtain the true kaon fraction, using the following transformation between the measured $\left(f_{i}\right)$ and true $\left(\hat{f}_{i}\right)$ particle fractions in the sample:

$$
\begin{gathered}
f_{K}=\hat{f_{K}}-w\left(p_{T}\right) \hat{f_{K}} \\
f_{\pi}=\hat{f_{\pi}}+w\left(p_{T}\right) \hat{f_{K}}
\end{gathered}
$$

where $w\left(p_{T}\right)$ is the fraction of kaons decaying in flight that are identified as pions, which is parametrized using $w\left(p_{T}\right)=a_{0}+a_{1} p_{T}$, where the values of the parameters $a_{0}, a_{1}$ are listed in Table 5.5.

In order to estimate the systematic uncertainty due to the effect of kaons decaying in flight, we introduce a prior constraint function in the likelihood fit, which constrains the values of $w_{0}$ and $w_{1}$ in the fitting procedure:

$$
\chi^{2}(w)=\sum\left(a_{i}-\hat{a_{i}}\right) V_{i j}^{-1}\left(a_{j}-\hat{a_{j}}\right)
$$

where $a_{i} \in\left\{a_{0}, a_{1}\right\}$ and $\hat{a_{i}}$ denotes the central values of the parameters listed in Table 5.5, and $V$ is the element of the $2 \times 2$ covariance matrix where the off-diagonal element is obtained using the correlation coefficient listed in Table 5.5. The statistical 
uncertainty in $a_{0}, a_{1}$ is propagated in the true kaon fraction using this procedure in the likelihood fit.

\subsection{Systematic error due to mis-identification of particle types}

The results of test studies described in Section 5.4 indicated the presence of a small bias induced in the measured particle fractions that manifests itself as the misidentification of particle types. In Section 5.5, we described a model that is used to account for this bias in the measured particle fractions. Using the model, we propose that the true particle fractions and the measured particle fractions are related using the following linear transformation:

$$
\left(\begin{array}{c}
f_{\pi} \\
f_{k} \\
f_{p}
\end{array}\right)=\left(\begin{array}{ccc}
\alpha_{\pi \pi} & \alpha_{k \pi} & \alpha_{p \pi} \\
\alpha_{\pi k} & \alpha_{k k} & \alpha_{p k} \\
\alpha_{\pi p} & \alpha_{k p} & \alpha_{p p}
\end{array}\right)\left(\begin{array}{c}
\hat{f}_{\pi} \\
\hat{f}_{k} \\
\hat{f}_{p}
\end{array}\right)
$$

where $\hat{f}_{i}$ is the true particle fraction of particle type $i$ and and $f_{i}$ is the measured particle fraction. The parameters $\alpha_{i j}$ give the estimate of the bias induced in the measured fraction of particle type $i$ due to the presence of particle type $j$ in the sample. The values of the bias-matrix elements obtained by studying pure pion and pure kaon samples are listed in tables 5.1 and 5.2.

In the multi-dimensional likelihood fit performed to extract the kaon fraction around the prompt $D_{s}^{ \pm} / D^{ \pm}$components in data, we apply a correction in the true particle fractions $\hat{f}_{\pi}, \hat{f}_{k}$ and $\hat{f}_{p}$ using the bias correction model:

$$
\begin{aligned}
& f_{\pi}=\alpha_{\pi \pi} \hat{f}_{\pi}+\alpha_{k \pi} \hat{f}_{k}+\alpha_{p \pi} \hat{f}_{p} \\
& f_{k}=\alpha_{\pi k} \hat{f}_{\pi}+\alpha_{k k} \hat{f}_{k}+\alpha_{p k} \hat{f}_{p} \\
& f_{p}=\alpha_{\pi p} \hat{f}_{\pi}+\alpha_{k p} \hat{f}_{k}+\alpha_{p p} \hat{f}_{p}
\end{aligned}
$$


We estimate the systematic uncertainty in the true particle fractions that results from propagating the statistical uncertainty in the bias-matrix elements. Ideally, the propagation can be done using a prior constraint function in the likelihood fit for the $\alpha_{i j}$ parameters. Since the parameters are obtained in ranges of $p_{T}$, we obtain a $3 \times 3$ matrix in each momentum range. Hence, we would need to include a prior constraint function in the likelihood fit for each momentum range, which makes the implementation of the procedure very complicated.

Instead, we opt to use a method that provides a reasonable estimate of the systematic uncertainty but is easier to implement. We calculate the systematic uncertainty in the measured kaon fraction by varying each of the $\alpha_{i j}$ parameters by $\pm 1 \sigma$ (statistical error) and perform a likelihood fit in each case. From the set of likelihood fits, we calculate the maximum positive and maximum negative variation in the measured kaon fraction. The maximum positive variation and the maximum negative variation is quoted as a systematic uncertainty.

\subsection{Systematic uncertainty due to TOF and $d E / d x$ parameterization}

We calibrate the TOF and $d E / d x$ response of the detector using control samples as described in Chapter 5. The parameters in the probability density functions used for calibration have statistical errors due to finite size of the control samples. We estimate the systematic uncertainty in the measured kaon fraction that results from propagating the statistical errors in the TOF and $d E / d x$ parameterization.

Ideally, the error propagation can be done using a prior constraint function in the likelihood fit for the parameters in the TOF and $d E / d x$ components. However, the combined particle identification parameterizations have 33 parameters that are obtained in ranges of transverse momentum, which makes the inclusion of a prior constraint function for a $33 \times 33$ matrix cumbersome. 
Instead, we use a method that provides a reasonable estimate of the systematic uncertainty but is easier to implement, which is similar to the procedure that was used to estimate the systematic uncertainty in Section 7.2:

- measure the kaon fraction $\left(f_{K}\right)$ around prompt $D_{s}^{ \pm}, D^{ \pm}$mesons,

- vary the parameters in the TOF and $d E / d x$ probability density functions by $\pm 1 \sigma$ (statistical uncertainty),

- re-measure the kaon fraction $\left(f_{K}^{\prime}\right)$ around prompt $D_{s}^{ \pm}, D^{ \pm}$mesons,

- calculate the variation $\Delta f_{K}$ in the measured kaon fraction for each parameter that is varied by $\pm 1 \sigma: \Delta f_{K}=f_{K}-f_{K}^{\prime}$,

- identify the maximum positive variation for TOF and $d E / d x$ template parameters: $\Delta f_{K}^{T O F \mid+}$ and $\Delta f_{K}^{d E / d x \mid+}$,

- Identify the maximum negative variation for TOF and $d E / d x$ template parameters: $\Delta f_{K}^{T O F \mid-}$ and $\Delta f_{K}^{d E / d x \mid-}$,

- The overall positive/negative variation in the measured kaon fraction is calculated using:

$$
\begin{aligned}
& \Delta f_{K}^{P I D \mid+}=\sqrt{\left(\Delta f_{K}^{T O F \mid+}\right)^{2}+\left(\Delta f_{K}^{d E / d x \mid+}\right)^{2}} \\
& \Delta f_{K}^{P I D \mid-}=\sqrt{\left(\Delta f_{K}^{T O F \mid-}\right)^{2}+\left(\Delta f_{K}^{d E / d x \mid-}\right)^{2}}
\end{aligned}
$$

The overall positive variation $\Delta f_{K}^{P I D \mid+}$ and negative variation $\Delta f_{K}^{P I D \mid-}$ is quoted as a systematic uncertainty in the measured kaon fraction that results from propagating the statistical error in the parametrization of the TOF and $d E / d x$ distributions.

\subsection{Systematic uncertainty due to parametrization of $K K \pi$ mass and $d_{0}$ distributions}

In order to measure particle fractions around prompt $D_{s}^{ \pm}, D^{ \pm}$mesons, we use the $K K \pi$ invariant mass and impact parameter distributions to separate the prompt 
$D_{s}^{ \pm} / D^{ \pm}$, secondary $D_{s}^{ \pm} / D^{ \pm}$and background components. The parameters in the probability distribution functions used to describe the shapes of the various components in these distributions have statistical uncertainties due to finite sizes of the Monte Carlo samples generated.

We estimate the systematic error in the measured kaon fraction that results by propagating the statistical uncertainty in the parameters of the invariant mass and impact parameter probability distribution functions. In this case as well, the inclusion of a prior constraint function in the likelihood fit is cumbersome since we have 35 parameters, from the mass and $d_{0}$ parameterizations in five ranges of $p_{T}(K K \pi)$. Using a similar procedure to the one used to evaluate the systematic uncertainty in Section 7.3, we assess the systematic uncertainty as follows:

- measure the kaon fraction $\left(f_{K}\right)$ around prompt $D_{s}^{ \pm}, D^{ \pm}$mesons,

- arrange the parameters into eight groups according to the components they describe in the invariant mass and impact parameter distributions,

- vary the parameters describing the shapes of the components in each group by $\pm 1 \sigma$ (statistical uncertainty),

- re-measure the kaon fraction $\left(f_{K}^{\prime}\right)$ around prompt $D_{s}^{ \pm}, D^{ \pm}$mesons,

- calculate the variation $\Delta f_{K}$ in the measured kaon fraction for each parameter that is varied by $\pm 1 \sigma: \Delta f_{K}=f_{K}-f_{K}^{\prime}$,

- identify the maximum positive variation $\Delta f_{K}^{K K \pi|+| g}$ for each group $g \in\{1,2 \ldots 8\}$ of parameters,

- identify the maximum negative variation $\Delta f_{K}^{K K \pi|-| g}$ for each group $g \in\{1,2 \ldots 8\}$ of parameters, 
- the maximum variations in all the groups are added in quadrature to calculate the overall positive/negative variation in the measured kaon fraction:

$$
\begin{aligned}
\Delta f_{K}^{K K \pi \mid+} & =\sqrt{\sum_{g}\left(\Delta f_{K}^{K K \pi|+| g}\right)^{2}} \\
\Delta f_{K}^{K K \pi \mid-} & =\sqrt{\sum_{g}\left(\Delta f_{K}^{K K \pi|-| g}\right)^{2}}
\end{aligned}
$$

where $g$ iterates over the eight groups of parameters listed in Table 7.1.

The overall positive $\Delta f_{K}^{K K \pi \mid+}$ and negative $\Delta f_{K}^{K K \pi \mid-}$ variation is quoted as a systematic uncertainty in the measured kaon fraction that results from propagating the statistical error in the parametrization of the $K K \pi$ invariant mass and impact parameter distributions.

Table 7.1: List of the groups in which the parameters describing the various components/features in data are sorted.

\begin{tabular}{|c|c|}
\hline Group & Variable/Component \\
\hline 1 & $D_{s}^{ \pm} / D^{ \pm}$signal peak \\
2 & Wide resonant structure \\
3 & Background in $M(K K \pi)$ distribution \\
4 & Prompt $d_{0}$ resolution \\
5 & Secondary $d_{0}$ distribution \\
6 & Background $d_{0}$ distribution \\
7 & $D_{s}^{ \pm} / D^{ \pm}$fraction in data \\
8 & Prompt fractions for $D_{s}^{ \pm} / D^{ \pm}$ \\
\hline
\end{tabular}

\subsection{Summary of systematic studies}

We studied various sources that can result in a systematic uncertainty in the measured kaon fraction such as: 
- mis-identification of particle types: systematic error is denoted by $\Delta f_{K}^{\text {Bias } \mid+}$ and $\Delta f_{K}^{\text {Bias } \mid-}$

- effect of kaons decaying in flight: systematic error is denoted by $\Delta f_{K}^{\text {decay }}$

- propagation of the statistical error in the parameters describing the TOF and $d E / d x$ probability density functions: systematic error is denoted by $\Delta f_{K}^{P I D \mid+}$ and $\Delta f_{K}^{P I D \mid-}$

- propagation of the statistical error in the parameters describing the shape of the various components in the $K^{+} K^{-} \pi$ invariant mass and impact parameter distributions: systematic error is denoted by $\Delta f_{K}^{K K \pi \mid+}$ and $\Delta f_{K}^{K K \pi \mid-}$

The total positive and negative systematic uncertainty in the measured kaon fraction is calculated by adding the contributions from the above sources in quadrature:

$$
\begin{aligned}
& \Delta f_{\text {syst }}^{+}=\sqrt{\left(\Delta f_{K}^{\text {decay }}\right)^{2}+\left(\Delta f_{K}^{K K \pi \mid+}\right)^{2}+\left(\Delta f_{K}^{P I D \mid+}\right)^{2}+\left(\Delta f_{K}^{\text {Bias } \mid+}\right)^{2}} \\
& \Delta f_{\text {syst }}^{-}=\sqrt{\left(\Delta f_{K}^{\text {decay }}\right)^{2}+\left(\Delta f_{K}^{K K \pi \mid-}\right)^{2}+\left(\Delta f_{K}^{\text {PID } \mid-}\right)^{2}+\left(\Delta f_{K}^{\text {Bias } \mid-}\right)^{2}}
\end{aligned}
$$

The results of the systematic studies indicate that the systematic uncertainties are generally small for low transverse momentum $p_{T}<2.0 \mathrm{GeV} /$ c. For higher momentum range $2.0<p_{T}<6.0 \mathrm{GeV} / \mathrm{c}$, the most significant systematic error is the mis-identification of particle types. The evaluated systematic uncertainties and the results of the analysis are discussed in the next chapter. 


\section{DISCUSSION OF RESULTS}

As described in the previous chapter, we perform a multidimensional likelihood fit to extract the fraction of kaons that are produced in association with prompt $D_{s}^{ \pm} / D^{ \pm}$ mesons. While performing the measurements, we pick the maximum- $p_{T}$ track in the cone around the $D_{s}^{ \pm} / D^{ \pm}$candidates, which is based on the hypothesis that the maximum- $p_{T}$ track is more likely to be correlated with the charm fragmentation process.

In addition, using the sample of maximum $p_{T}$ tracks (one track per $D_{s}^{ \pm} / D^{ \pm}$candidate) reduces the ambiguity in interpreting the results. If we were to include all the tracks found in the cone around a candidate, the measured kaon fraction would depend on the number of other tracks of various types found in the cone. Picking the maximum- $p_{T}$ track solves this problem, since we analyze at most one track per candidate in the cone around each candidate.

We measure the kaon fraction around prompt $D_{s}^{ \pm} / D^{ \pm}$mesons in ranges of three kinematic variables that are described below. The first variable we select is the transverse momentum, $p_{T}$, of the maximum- $p_{T}$ track in the cone. The $D_{s}^{ \pm}$mesons that are produced via the fragmentation of a charm quark have high transverse momentum. When a charm quark combines with a strange/anti-strange quark to produce a $D_{s}^{ \pm}$ meson, an anti-strange/strange quark will be left from the $s \bar{s}$ pair created in the fragmentation process. This remaining anti-strange/strange quark will hadronizes to produce a kaon and is typically expected to have higher $p_{T}$ than other particles produced in the fragmentation process. In general, the $p_{T}$ of the particle produced in the second branching of a heavy quark fragmentation process will be correlated with the $p_{T}$ of the heavy meson that is created in the process. Hence, transverse momentum is a basic kinematic quantity that can be used to study correlations between heavy mesons and particles produced in the second branching. 
The second variable chosen for the study is the invariant mass of the $D_{s}^{ \pm}$or $D^{ \pm}$ candidate and the maximum- $p_{T}$ track in the cone, assuming the kaon mass hypothesis, $m_{D K}$, calculated using:

$$
m_{D K}=\sqrt{\left(E_{D}+E_{K}\right)^{2}-\left|\vec{p}_{D}+\vec{p}_{K}\right|^{2}}
$$

where $E_{D}$ and $\vec{p}_{D}$ are the energy and momentum of the $D$ candidate, and $\vec{p}_{K}$ and $E_{K}$ are the momentum and energy of the maximum- $p_{T}$ track in the cone. The $m_{D K}$ variable is a good choice for the comparative study since it is Lorentz invariant.

And Lastly, we measure the kaon fraction in ranges of the rapidity difference between the $K K \pi$ candidate and the maximum- $p_{T}$ track in the cone, along the candidate momentum axis, $\Delta y=y_{K}-y_{D}$, where $y_{K}$ is the rapidity of the kaon and $y_{D}$ is the rapidity of the $D$ meson candidate:

$$
\begin{aligned}
& y_{D}=\frac{1}{2} \ln \left(\frac{E_{D}+p_{D}}{E_{D}-p_{D}}\right) \\
& y_{K}=\frac{1}{2} \ln \left(\frac{E_{K}+p_{\|}}{E_{K}-p_{\|}}\right)
\end{aligned}
$$

where $p_{\|}$is the component of the track momentum parallel to the direction of the $D$ momentum vector $\hat{p}_{D}$. The difference of $D_{s}^{ \pm}$rapidity and kaon rapidity along the fragmentation axis would have been an ideal variable for comparison, however, in practice it is not possible to extract the direction of the fragmentation axis, hence we use the direction of the $D$ momentum vector as an approximation for the fragmentation axis.

In addition, we measure the kaon fraction separately for $D_{s}^{ \pm} K^{\mp}$ (opposite sign) and $D_{s}^{ \pm} K^{ \pm}$(same sign) combinations. In the opposite sign combination, we expect the kaon production to be enhanced around prompt $D_{s}^{ \pm}$compared to prompt $D^{ \pm}$, since formation of a prompt $D_{s}^{ \pm}$meson requires conservation of strangeness in the first fragmentation branch as shown in Figure 1.1. In the same sign combination, we expect the kaon production to be similar around both $D_{s}^{ \pm} / D^{ \pm}$mesons, since same sign kaons are likely to be produced after the second branch in the fragmentation process as illustrated in Figure 1.1. The fraction of opposite sign kaons around prompt $D^{ \pm}$ may be expected to be similar to the fraction of same sign kaons around prompt $D_{s}^{ \pm}$, 
since both types of kaons are produced later in the fragmentation process and are not directly correlated to the fragmentation of the heavy quark.

In the analysis presented in this dissertation, we compare the results in data with predictions of fragmentation models used in the PYTHIA and HERWIG event generators. The PYTHIA event generator uses the string fragmentation model, whereas the cluster fragmentation model is used in the HERWIG event generator, which are described in Chapter 3. Using PYTHIA version 8.1 [38] and HERWIG version 6.5 [39], we generate $c \bar{c}$ events and require the $D_{s}^{ \pm} / D^{ \pm}$mesons to be stable particles by disabling $D_{s}^{ \pm} / D^{ \pm}$decays in the simulations. In order to extract the kaon fraction around $D_{s}^{ \pm} / D^{ \pm}$mesons in Monte Carlo events, we identify $D_{s}^{ \pm}$or $D^{ \pm}$mesons and then measure the kaon fraction in the maximum- $p_{T}$ track sample selected from tracks in a cone of radius $\Delta R<0.7$ around the $D_{s}^{ \pm}$or $D^{ \pm}$mesons found in the events.

The measured kaon fraction around prompt $D_{s}^{ \pm} / D^{ \pm}$mesons, in ranges of $p_{T}, m_{D K}$ and $\Delta y$ are shown in figures 8.1, 8.2 and 8.3 respectively. In addition, the figures also show the kaon fraction measured in the two charge combinations, separately for both prompt $D_{s}^{ \pm}$and $D^{ \pm}$mesons, and a comparison between the results and predictions of the PYTHIA and HERWIG event generators. Tables 8.1-8.12 list the values of the measured kaon fraction around prompt $D_{s}^{ \pm} / D^{ \pm}$mesons, measured in the two sign scenarios and in ranges of the three kinematic variables.

Comparing the graphs marked $(a)$ and $(b)$ in Figures 8.1, 8.2 and 8.3, we can see that kaon production around prompt $D_{s}^{ \pm}$is enhanced compared to prompt $D^{ \pm}$, in the opposite sign combination. This result is consistent with the naive fragmentation picture, illustrated in Figure 1.1, in which opposite sign kaons are produced in the second branch of the fragmentation process around prompt $D_{s}^{ \pm}$mesons and not around prompt $D^{ \pm}$mesons. Hence, we can conclude that opposite sign kaons produced around prompt $D_{s}^{ \pm}$mesons provide information about the $p_{T}, m_{D K}$ and $\Delta y$ spectrum of hadrons produced in the second fragmentation branching.

Moreover, the results in graph marked (b) show that the production of opposite sign kaons around prompt $D^{ \pm}$mesons is suppressed, as one might expect, since these 
kaons can be produced only after the third branch in the fragmentation process as illustrated in Figure 1.1. The graphs marked $(c)$ and $(d)$ in figures 8.1-8.3 indicate that in the same sign charge combination, the measured kaon fraction around both prompt $D_{s}^{ \pm}$and prompt $D^{ \pm}$mesons is similar, which is expected since same sign kaons are likely to be produced in the third branch around both mesons as shown in Figure 1.1.

As shown in Figure 8.1, a comparison between the results and predictions of the models indicates that the enhanced kaon fraction for the leading opposite sign particle (graph $a$ ), is described by the models. While we observe slightly higher kaon fraction for particles produced in subsequent fragmentation branches, compared to the predictions of the models (graphs $b, c$ and $d$ ). However, in figures 8.2 and 8.3, the measured fraction for kaons produced after the second branching (graphs $b, c$ and $d$ ) seems to be in better qualitative agreement with predictions of the models, compared to the fraction of kaons for particles produced in the second branching (graph $a$ ).

In conclusion, a comparison of the results with predictions of the string fragmentation model (used in PYTHIA) and the cluster fragmentation model (used in HERWIG) shows that the observed enhancement of opposite sign kaons that are likely to be produced in the second fragmentation branch, is also a feature implemented in the models. This enhancement of opposite sign kaons is observed in all three kinematic variables, namely $p_{T}, m_{D K}$ and $\Delta y$. We observe interesting differences in some distributions, however, it is un-obvious whether it would be feasible to tune the parameters in the fragmentation models such that the models provide a description of all the features, at various levels of branching, simultaneously. 

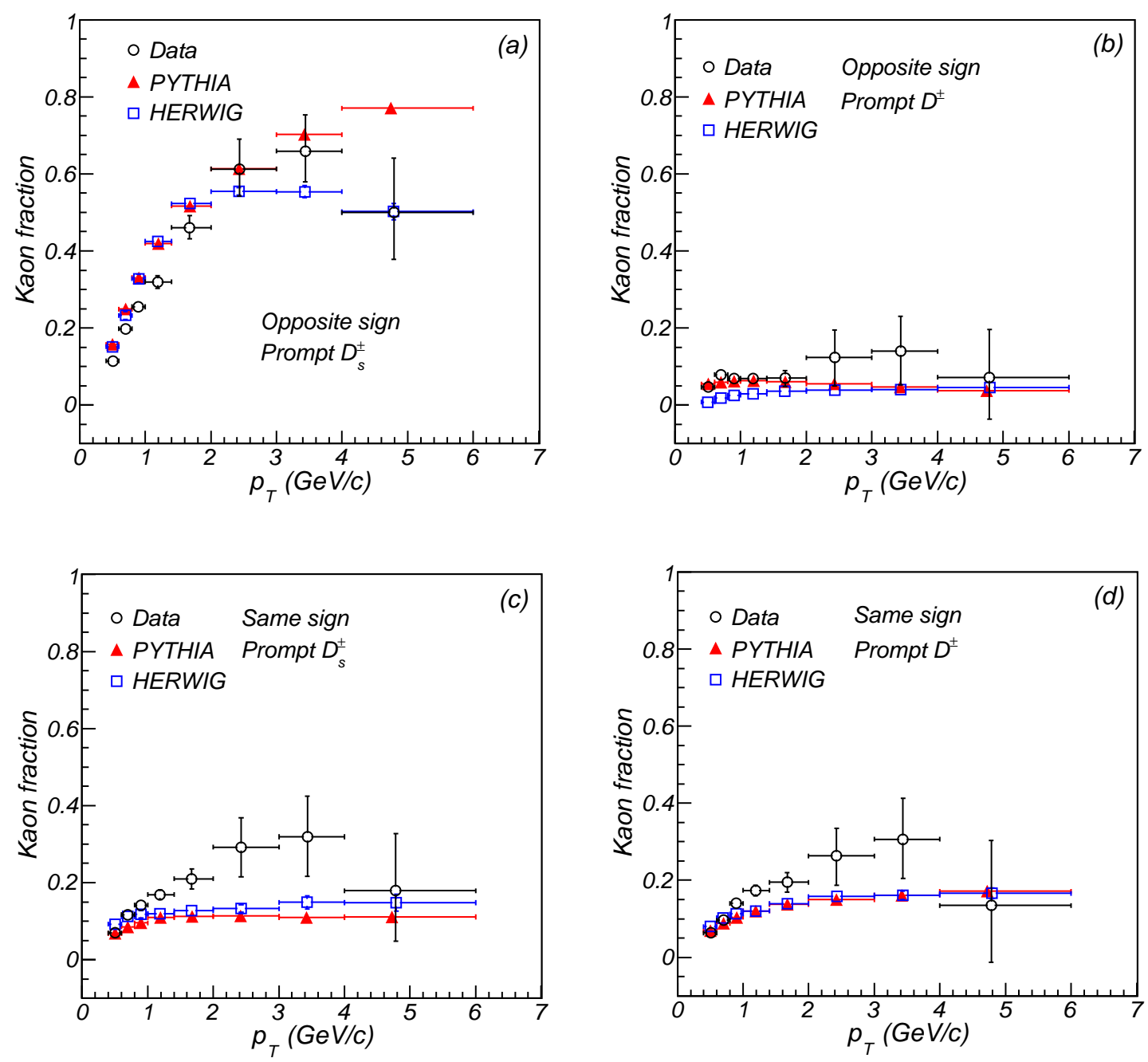

Figure 8.1.: Kaon fraction measured around prompt $D_{s}^{ \pm} / D^{ \pm}$mesons in ranges of transverse momentum of the maximum- $p_{T}$ track found in the cone. 

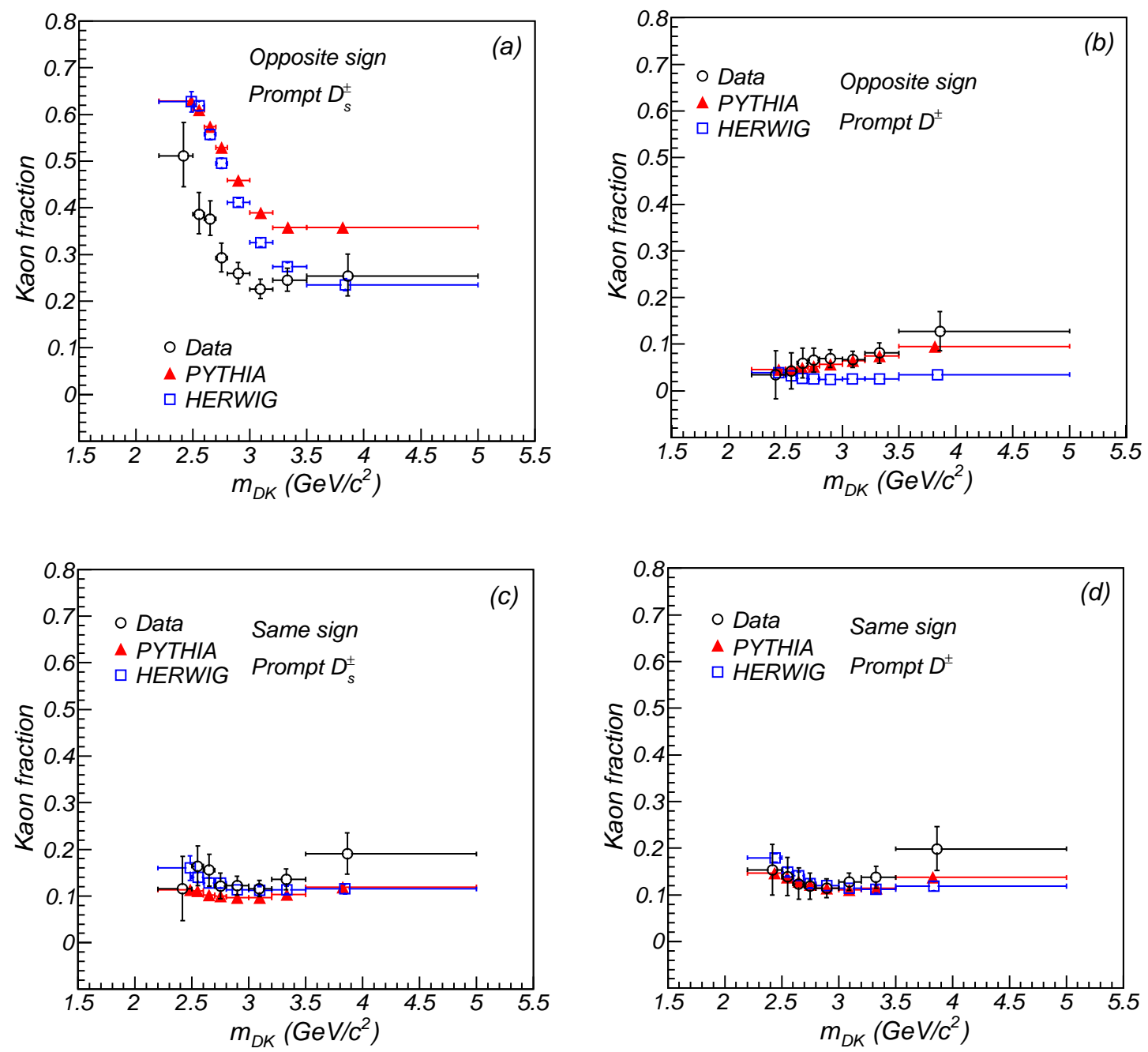

Figure 8.2.: Kaon fraction measured around prompt $D_{s}^{ \pm} / D^{ \pm}$mesons in ranges of invariant mass of the the maximum- $p_{T}$ track in the cone and the $D$ candidate. In graph $(a)$, the kaon fraction peaks around $m_{D K}=2.46 \mathrm{GeV} / \mathrm{c}^{2}$, which corresponds to correct mass hypothesis in cases where the maximum- $p_{T}$ track is likely to be an opposite sign kaon produced in the second branch. 

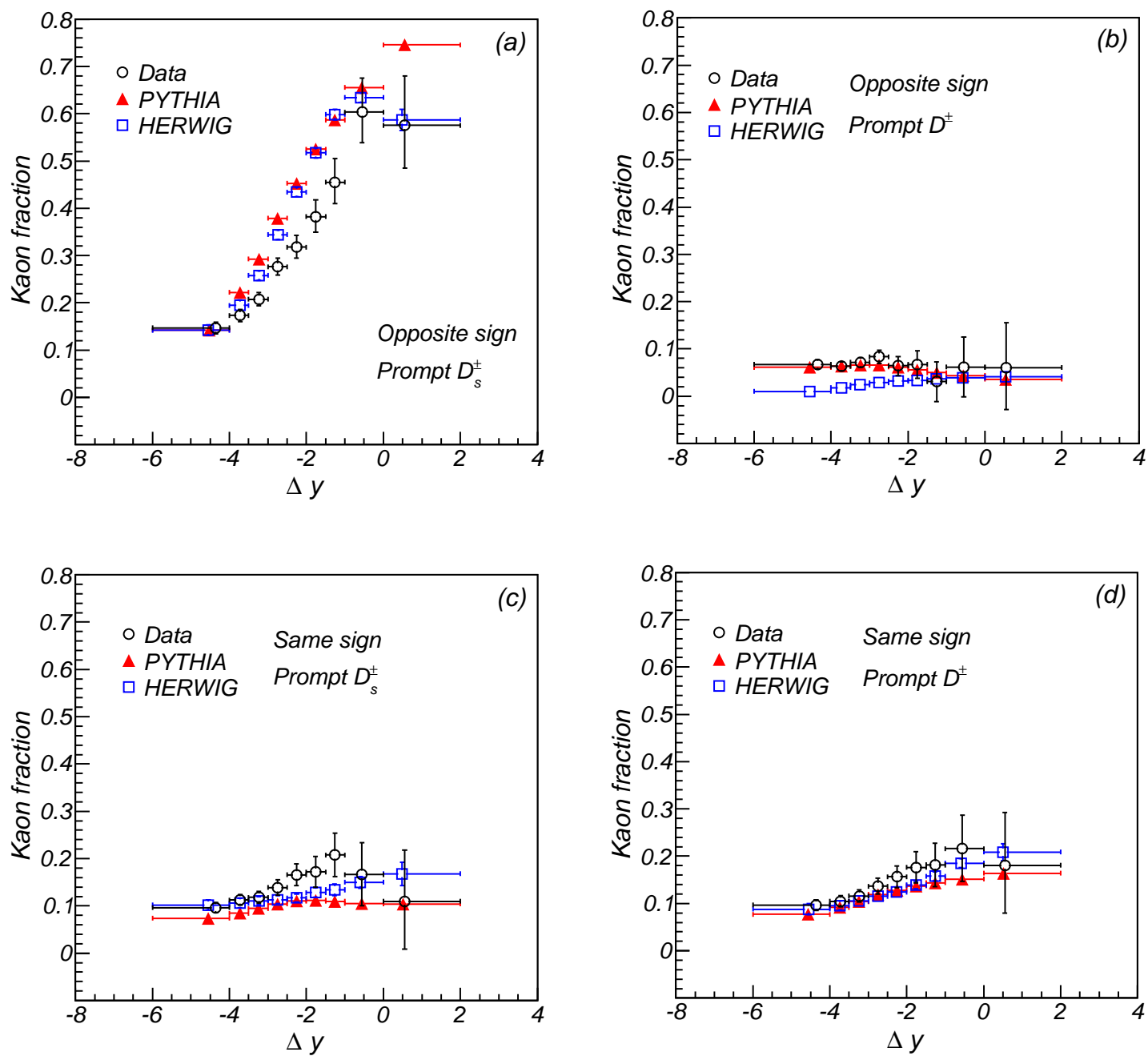

Figure 8.3.: Kaon fraction measured around prompt $D_{s}^{ \pm} / D^{ \pm}$mesons in ranges of rapidity difference between the maximum- $p_{T}$ track and the $D$ candidate along the $D$ momentum axis. In graph $(a)$, the kaon fraction in the opposite sign combination peaks around $\Delta y=0$, which implies that the maximum- $p_{T}$ track with almost similar rapidity as compared to the $D_{s}^{ \pm}$meson is more likely to be a kaon. 
Table 8.1: Measured kaon fraction around prompt $D_{s}^{ \pm}$and prompt $D^{ \pm}$mesons in ranges of transverse momentum $\left(p_{T}\right)$ of the maximum- $p_{T}$ track found in the cone, for the opposite sign charge combination. The first uncertainty is statistical and the second is the combination of all the systematic uncertainties.

\begin{tabular}{|c|c|c|}
\hline $\begin{array}{l}p_{T} \text { range } \\
(\mathrm{GeV} / \mathrm{c})\end{array}$ & $\begin{array}{l}\text { Opposite sign kaon fraction } \\
\text { around prompt } D_{s}^{ \pm}\end{array}$ & $\begin{array}{c}\text { Opposite sign kaon fraction } \\
\text { around prompt } D^{ \pm}\end{array}$ \\
\hline $0.4-0.6$ & $0.1142 \pm 0.0050_{-0.0057}^{+0.0053}$ & $0.0463 \pm 0.0043{ }_{-0.0027}^{+0.0025}$ \\
\hline $0.6-0.8$ & $0.1978 \pm 0.00633_{-0.0021}^{+0.0022}$ & $0.0788 \pm 0.0056_{-0.0014}^{+0.0015}$ \\
\hline $0.8-1.0$ & $0.2550 \pm 0.0079_{-0.0024}^{+0.0025}$ & $0.0685 \pm 0.0067_{-0.0014}^{+0.0015}$ \\
\hline $1.0-1.4$ & $0.320 \pm 0.008_{-0.015}^{+0.014}$ & $0.0687 \pm 0.00633_{-0.0066}^{+0.0060}$ \\
\hline $1.4-2.0$ & $0.461 \pm 0.011_{-0.027}^{+0.028}$ & $0.070 \pm 0.010_{-0.019}^{+0.017}$ \\
\hline $2.0-3.0$ & $0.613 \pm 0.018_{-0.068}^{+0.075}$ & $0.123 \pm 0.015_{-0.072}^{+0.069}$ \\
\hline $3.0-4.0$ & $0.659 \pm 0.025_{-0.076}^{+0.091}$ & $0.139 \pm 0.024_{-0.084}^{+0.087}$ \\
\hline $4.0-6.0$ & $0.50 \pm 0.066_{-0.11}^{+0.13}$ & $0.07 \pm 0.05_{-0.10}^{+0.11}$ \\
\hline
\end{tabular}


Table 8.2: Measured kaon fraction around prompt $D_{s}^{ \pm}$and prompt $D^{ \pm}$mesons in ranges of transverse momentum $\left(p_{T}\right)$ of the maximum- $p_{T}$ track found in the cone, for the same sign charge combination. The first uncertainty is statistical and the second is the combination of all the systematic uncertainties.

\begin{tabular}{|c|c|c|}
\hline $\begin{array}{l}p_{T} \text { range } \\
(\mathrm{GeV} / \mathrm{c})\end{array}$ & $\begin{array}{l}\text { Same sign kaon fraction } \\
\text { around prompt } D_{s}^{ \pm}\end{array}$ & $\begin{array}{c}\text { Same sign kaon fraction } \\
\text { around prompt } D^{ \pm}\end{array}$ \\
\hline $0.4-0.6$ & $0.0695 \pm 0.0047_{-0.0033}^{+0.0031}$ & $0.0632 \pm 0.0057_{-0.0030}^{+0.0027}$ \\
\hline $0.6-0.8$ & $0.1170 \pm 0.0060_{-0.0015}^{+0.0016}$ & $0.0960 \pm 0.00688_{-0.0015}^{+0.0015}$ \\
\hline $0.8-1.0$ & $0.1414 \pm 0.0077_{-0.0017}^{+0.0019}$ & $0.1401 \pm 0.00899_{-0.0019}^{+0.0021}$ \\
\hline $1.0-1.4$ & $0.1692 \pm 0.0081_{-0.0093}^{+0.0089}$ & $0.1736 \pm 0.0091{ }_{-0.0097}^{+0.0093}$ \\
\hline $1.4-2.0$ & $0.210 \pm 0.013_{-0.023}^{+0.022}$ & $0.195 \pm 0.012_{-0.023}^{+0.022}$ \\
\hline $2.0-3.0$ & $0.292 \pm 0.022_{-0.074}^{+0.074}$ & $0.263 \pm 0.022_{-0.073}^{+0.069}$ \\
\hline $3.0-4.0$ & $0.319 \pm 0.055_{-0.086}^{+0.090}$ & $0.306 \pm 0.051_{-0.089}^{+0.094}$ \\
\hline $4.0-6.0$ & $0.18 \pm 0.05_{-0.12}^{+0.14}$ & $0.13 \pm 0.099_{-0.12}^{+0.14}$ \\
\hline
\end{tabular}


Table 8.3: Measured kaon fraction $\left(f_{K}\right)$ around prompt $D_{s}^{ \pm}$and prompt $D^{ \pm}$mesons in ranges of transverse momentum $\left(p_{T}\right)$ of the maximum- $p_{T}$ track found in the cone, for the opposite sign charge combination. The first uncertainty is the statistical error (stat) followed by the systematic uncertainties due to sources such as: Effect of kaons decaying in flight (DIF), probability density functions used to separate prompt and secondary D components (PDF), calibration of Time-of-Flight and $d E / d x$ distributions for particle identification (calib) and mis-identification of particle species (mis-ID).

\begin{tabular}{|c|c|c|c|c|c|c|c|c|c|c|c|c|}
\hline $\begin{array}{l}p_{T} \text { range } \\
(\mathrm{GeV} / \mathrm{c})\end{array}$ & \multicolumn{6}{|c|}{$\begin{array}{l}\text { Opposite sign kaon fraction } \\
\text { around prompt } D_{s}^{ \pm}\end{array}$} & \multicolumn{6}{|c|}{$\begin{array}{l}\text { Opposite sign kaon fraction } \\
\text { around prompt } D^{ \pm}\end{array}$} \\
\hline $0.4-0.6$ & 0.1142 & \pm 0.0050 & \pm 0.0006 & $\begin{array}{l}+0.0004 \\
-0.0004\end{array}$ & $\begin{array}{l}+0.0010 \\
-0.0010\end{array}$ & $\begin{array}{l}+0.0052 \\
-0.0056\end{array}$ & 0.0463 & \pm 0.0043 & \pm 0.0012 & $\begin{array}{l}+0.0003 \\
-0.0003\end{array}$ & $\begin{array}{l}+0.0006 \\
-0.0006\end{array}$ & $\begin{array}{l}+0.0021 \\
-0.0023\end{array}$ \\
\hline $0.6-0.8$ & 0.1978 & \pm 0.0063 & \pm 0.0011 & $\begin{array}{l}+0.0006 \\
-0.0007\end{array}$ & $\begin{array}{l}+0.0017 \\
-0.0015\end{array}$ & $\begin{array}{l}+0.0008 \\
-0.0008\end{array}$ & 0.0788 & \pm 0.0056 & \pm 0.0007 & $\begin{array}{l}+0.0006 \\
-0.0005\end{array}$ & $\begin{array}{l}+0.0011 \\
-0.0010\end{array}$ & $\begin{array}{l}+0.0003 \\
-0.0003\end{array}$ \\
\hline $0.8-1.0$ & 0.2550 & \pm 0.0079 & \pm 0.0014 & $\begin{array}{l}+0.0011 \\
-0.0011\end{array}$ & $\begin{array}{l}+0.0014 \\
-0.0011\end{array}$ & $\begin{array}{l}+0.0010 \\
-0.0010\end{array}$ & 0.0685 & \pm 0.0067 & \pm 0.0001 & $\begin{array}{l}+0.0006 \\
-0.0006\end{array}$ & $\begin{array}{l}+0.0014 \\
-0.0012\end{array}$ & $\begin{array}{l}+0.0003 \\
-0.0004\end{array}$ \\
\hline $1.0-1.4$ & 0.320 & \pm 0.008 & \pm 0.001 & $\begin{array}{l}+0.001 \\
{ }_{-0.001}\end{array}$ & $\begin{array}{l}+0.005 \\
-0.005\end{array}$ & $\begin{array}{l}+0.013 \\
-0.014\end{array}$ & 0.0687 & \pm 0.0063 & \pm 0.0010 & $\begin{array}{l}+0.0005 \\
-0.0011\end{array}$ & $\begin{array}{l}+0.0033 \\
-0.0037\end{array}$ & $\begin{array}{l}+0.0049 \\
-0.0053\end{array}$ \\
\hline $1.4-2.0$ & 0.461 & \pm 0.011 & \pm 0.003 & $\begin{array}{l}+0.002 \\
-0.002\end{array}$ & $\begin{array}{l}+0.028 \\
-0.027\end{array}$ & $\begin{array}{l}+0.004 \\
-0.004\end{array}$ & 0.070 & \pm 0.010 & \pm 0.000 & $\begin{array}{l}+0.002 \\
-0.002\end{array}$ & $\begin{array}{l}+0.017 \\
-0.018\end{array}$ & $\begin{array}{l}+0.003 \\
-0.003\end{array}$ \\
\hline $2.0-3.0$ & 0.613 & \pm 0.018 & \pm 0.002 & $\begin{array}{l}+0.003 \\
-0.003\end{array}$ & $\begin{array}{l}+0.060 \\
-0.055\end{array}$ & $\begin{array}{l}+0.044 \\
-0.040\end{array}$ & 0.123 & \pm 0.015 & \pm 0.003 & $\begin{array}{l}+0.002 \\
-0.002\end{array}$ & $\begin{array}{l}+0.041 \\
-0.050\end{array}$ & $\begin{array}{l}+0.055 \\
-0.051\end{array}$ \\
\hline $3.0-4.0$ & 0.659 & \pm 0.025 & \pm 0.008 & $\begin{array}{l}+0.004 \\
-0.004\end{array}$ & $\begin{array}{l}+0.066 \\
-0.055\end{array}$ & $\begin{array}{l}+0.061 \\
-0.052\end{array}$ & 0.139 & \pm 0.024 & \pm 0.005 & $\begin{array}{l}+0.007 \\
-0.005\end{array}$ & $\begin{array}{l}+0.054 \\
-0.053\end{array}$ & $\begin{array}{l}+0.068 \\
-0.065\end{array}$ \\
\hline $4.0-6.0$ & 0.50 & \pm 0.06 & \pm 0.01 & $\begin{array}{l}+0.00 \\
-0.00 \\
\end{array}$ & $\begin{array}{l}+0.07 \\
-0.05 \\
\end{array}$ & $\begin{array}{l}+0.11 \\
-0.09 \\
\end{array}$ & 0.072 & \pm 0.051 & \pm 0.005 & $\begin{array}{l}+0.007 \\
-0.005 \\
\end{array}$ & $\begin{array}{l}+0.053 \\
-0.053 \\
\end{array}$ & $\begin{array}{l}+0.098 \\
-0.080 \\
\end{array}$ \\
\hline
\end{tabular}


Table 8.4: Measured kaon fraction $\left(f_{K}\right)$ around prompt $D_{s}^{ \pm}$and prompt $D^{ \pm}$mesons in ranges of transverse momentum $\left(p_{T}\right)$ of the maximum- $p_{T}$ track found in the cone, for the same sign charge combination. The first uncertainty is the statistical error (stat) followed by the systematic uncertainties due to sources such as: Effect of kaons decaying in flight (DIF), probability density functions used to separate prompt and secondary D components (PDF), calibration of Time-of-Flight and $d E / d x$ distributions for particle identification (calib) and mis-identification of particle species (mis-ID).

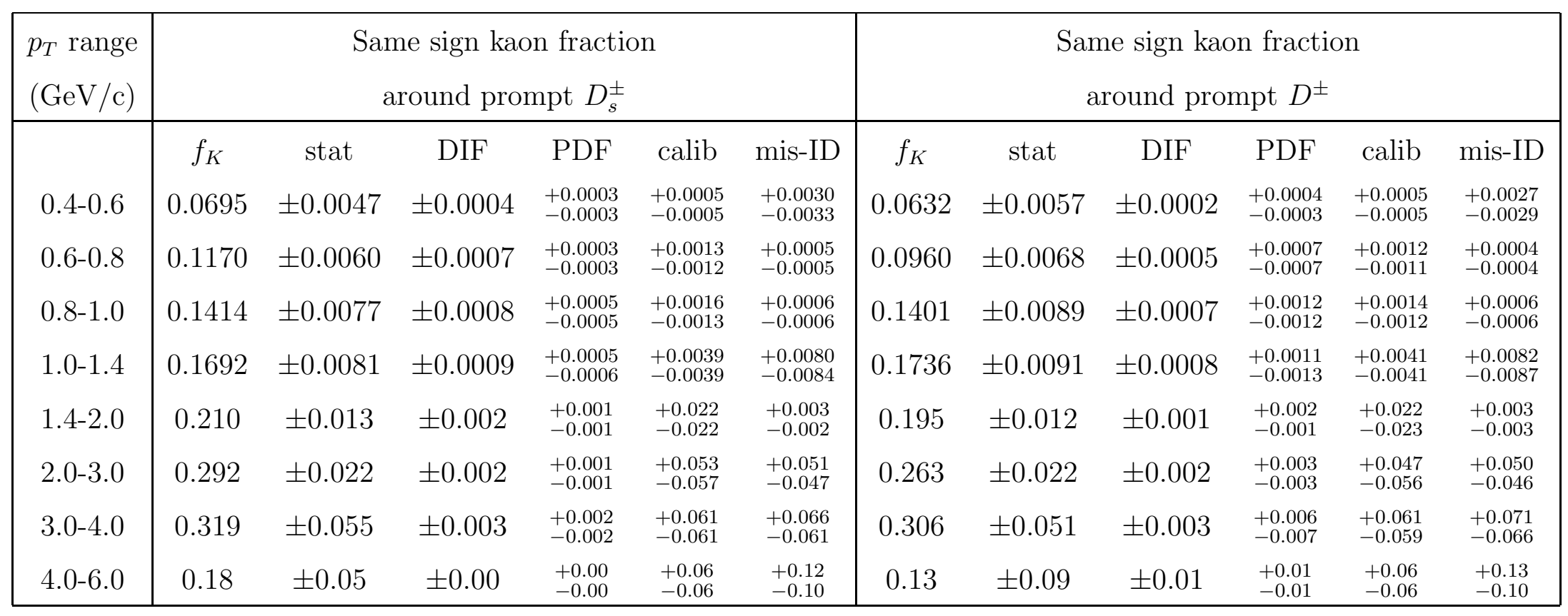


Table 8.5: Measured kaon fraction around prompt $D_{s}^{ \pm}$and prompt $D^{ \pm}$mesons in ranges of the invariant mass of the $\mathrm{D}$ candidate and the maximum- $p_{T}$ track found in the cone $\left(m_{D K}\right)$, for the opposite sign charge combination. The first uncertainty is statistical and the second is the combination of all the systematic uncertainties.

\begin{tabular}{|c|c|c|}
\hline $\begin{array}{c}m_{D K} \text { range } \\
\left(\mathrm{GeV} / \mathrm{c}^{2}\right)\end{array}$ & $\begin{array}{l}\text { Opposite sign kaon fraction } \\
\text { around prompt } D_{s}^{ \pm}\end{array}$ & $\begin{array}{c}\text { Opposite sign kaon fraction } \\
\text { around prompt } D^{ \pm}\end{array}$ \\
\hline $2.2-2.5$ & $0.512 \pm 0.041_{-0.052}^{+0.059}$ & $0.034 \pm 0.011_{-0.050}^{+0.051}$ \\
\hline $2.5-2.6$ & $0.386 \pm 0.015_{-0.040}^{+0.044}$ & $0.043 \pm 0.009_{-0.037}^{+0.038}$ \\
\hline $2.6-2.7$ & $0.376 \pm 0.012{ }_{-0.033}^{+0.037}$ & $0.060 \pm 0.008_{-0.031}^{+0.031}$ \\
\hline $2.7-2.8$ & $0.292 \pm 0.010_{-0.028}^{+0.031}$ & $0.066 \pm 0.007_{-0.025}^{+0.025}$ \\
\hline $2.8-3.0$ & $0.259 \pm 0.007_{-0.021}^{+0.023}$ & $0.069 \pm 0.005_{-0.019}^{+0.019}$ \\
\hline $3.0-3.2$ & $0.225 \pm 0.007_{-0.019}^{+0.020}$ & $0.067 \pm 0.007_{-0.016}^{+0.016}$ \\
\hline $3.2-3.5$ & $0.245 \pm 0.009_{-0.022}^{+0.025}$ & $0.081 \pm 0.010_{-0.020}^{+0.020}$ \\
\hline $3.5-5.0$ & $0.254 \pm 0.013_{-0.040}^{+0.046}$ & $0.128 \pm 0.015_{-0.039}^{+0.040}$ \\
\hline
\end{tabular}


Table 8.6: Measured kaon fraction around prompt $D_{s}^{ \pm}$and prompt $D^{ \pm}$mesons in ranges of the invariant mass of the $\mathrm{D}$ candidate and the maximum- $p_{T}$ track found in the cone $\left(m_{D K}\right)$, for the same sign charge combination. The first uncertainty is statistical and the second is the combination of all the systematic uncertainties.

\begin{tabular}{|c|c|c|}
\hline $\begin{array}{c}m_{D K} \text { range } \\
\left(\mathrm{GeV} / \mathrm{c}^{2}\right)\end{array}$ & $\begin{array}{l}\text { Same sign kaon fraction } \\
\text { around prompt } D_{s}^{ \pm}\end{array}$ & $\begin{array}{c}\text { Same sign kaon fraction } \\
\text { around prompt } D^{ \pm}\end{array}$ \\
\hline $2.2-2.5$ & $0.115 \pm 0.046_{-0.051}^{+0.053}$ & $0.154 \pm 0.017_{-0.051}^{+0.052}$ \\
\hline $2.5-2.6$ & $0.164 \pm 0.017_{-0.039}^{+0.040}$ & $0.139 \pm 0.012_{-0.038}^{+0.039}$ \\
\hline $2.6-2.7$ & $0.155 \pm 0.012_{-0.032}^{+0.032}$ & $0.124 \pm 0.011_{-0.031}^{+0.032}$ \\
\hline $2.7-2.8$ & $0.121 \pm 0.0099_{-0.025}^{+0.026}$ & $0.118 \pm 0.010_{-0.025}^{+0.026}$ \\
\hline $2.8-3.0$ & $0.122 \pm 0.006_{-0.019}^{+0.019}$ & $0.114 \pm 0.007_{-0.019}^{+0.019}$ \\
\hline $3.0-3.2$ & $0.116 \pm 0.006_{-0.016}^{+0.016}$ & $0.128 \pm 0.0099_{-0.016}^{+0.016}$ \\
\hline $3.2-3.5$ & $0.136 \pm 0.008_{-0.020}^{+0.021}$ & $0.138 \pm 0.012_{-0.020}^{+0.020}$ \\
\hline $3.5-5.0$ & $0.190 \pm 0.013_{-0.042}^{+0.043}$ & $0.199 \pm 0.020_{-0.041}^{+0.043}$ \\
\hline
\end{tabular}


Table 8.7: Measured kaon fraction $\left(f_{K}\right)$ around prompt $D_{s}^{ \pm}$and prompt $D^{ \pm}$mesons in ranges of the invariant mass of the $\mathrm{D}$ candidate and the maximum- $p_{T}$ track found in the cone $\left(m_{D K}\right)$, for the opposite sign charge combination. The first uncertainty is the statistical error (stat) followed by the systematic uncertainties due to sources such as: Effect of kaons decaying in flight (DIF), probability density functions used to separate prompt and secondary D components (PDF), calibration of Time-of-Flight and $d E / d x$ distributions for particle identification (calib) and mis-identification of particle species (mis-ID).

\begin{tabular}{|c|c|c|c|c|c|c|c|c|c|c|c|c|}
\hline \multirow[t]{2}{*}{$\begin{array}{c}m_{D K} \text { range } \\
\left(\mathrm{GeV} / \mathrm{c}^{2}\right)\end{array}$} & \multicolumn{6}{|c|}{$\begin{array}{l}\text { Opposite sign kaon fraction } \\
\text { around prompt } D_{s}^{ \pm}\end{array}$} & \multicolumn{6}{|c|}{$\begin{array}{l}\text { Opposite sign kaon fraction } \\
\text { around prompt } D^{ \pm}\end{array}$} \\
\hline & $f_{K}$ & stat & DIF & PDF & calib & mis-ID & $f_{K}$ & stat & DIF & PDF & calib & mis-ID \\
\hline $2.2-2.5$ & 0.512 & \pm 0.041 & \pm 0.010 & $\begin{array}{l}+0.003 \\
{ }_{-0.003}\end{array}$ & ${ }_{-0.039}^{+0.044}$ & $\begin{array}{l}+0.038 \\
{ }_{-0.033}\end{array}$ & 0.034 & \pm 0.011 & \pm 0.005 & ${ }_{-0.002}^{+0.003}$ & ${ }_{-0.035}^{+0.032}$ & $\begin{array}{l}+0.039 \\
{ }_{-0.036}\end{array}$ \\
\hline $2.5-2.6$ & 0.386 & \pm 0.015 & \pm 0.002 & $\begin{array}{l}+0.002 \\
{ }_{-0.002}\end{array}$ & $\begin{array}{l}+0.034 \\
-0.030\end{array}$ & $\begin{array}{l}+0.029 \\
-0.026\end{array}$ & 0.043 & \pm 0.009 & \pm 0.002 & $\begin{array}{l}+0.002 \\
-0.002\end{array}$ & $\begin{array}{l}+0.024 \\
-0.026\end{array}$ & $\begin{array}{l}+0.029 \\
-0.027\end{array}$ \\
\hline $2.6-2.7$ & 0.376 & \pm 0.012 & \pm 0.002 & $\begin{array}{l}{ }_{-0.002}^{+0.002} \\
\end{array}$ & $\begin{array}{l}+0.028 \\
{ }_{-0.025}\end{array}$ & $\begin{array}{l}+0.025 \\
{ }_{-0.022}\end{array}$ & 0.060 & \pm 0.008 & \pm 0.000 & ${ }_{-0.002}^{+0.002}$ & & \\
\hline $2.7-2.8$ & 0.292 & \pm 0.010 & \pm 0.001 & $\begin{array}{l}{ }_{-0.002}^{+0.002} \\
\end{array}$ & $\begin{array}{l}+0.023 \\
{ }_{-0.021}\end{array}$ & $\begin{array}{l}+0.020 \\
-0.018\end{array}$ & 0.066 & \pm 0.007 & \pm 0.001 & $\begin{array}{l}{ }_{-0.002}^{+0.002} \\
-0.00\end{array}$ & $\begin{array}{l}+0.016 \\
-0.018\end{array}$ & \\
\hline 2.8-3.0 & 0.259 & \pm 0.007 & \pm 0.001 & $\begin{array}{l}+0.002 \\
{ }_{-0.002}\end{array}$ & $\begin{array}{l}+0.017 \\
-0.015\end{array}$ & $\begin{array}{l}+0.016 \\
-0.014\end{array}$ & 0.069 & \pm 0.005 & \pm 0.000 & ${ }_{-0.001}^{+0.001}$ & ${ }_{-0.013}^{+0.012}$ & $\begin{array}{l}+0.014 \\
-0.013\end{array}$ \\
\hline $3.0-3.2$ & 0.225 & \pm 0.007 & \pm 0.001 & $\begin{array}{l}+0.001 \\
-0.001\end{array}$ & $\begin{array}{l}+0.015 \\
-0.014\end{array}$ & $\begin{array}{l}+0.014 \\
-0.013\end{array}$ & 0.067 & \pm 0.007 & \pm 0.000 & $\begin{array}{l}+0.001 \\
-0.001\end{array}$ & & \\
\hline $3.2-3.5$ & 0.245 & \pm 0.009 & \pm 0.001 & $\begin{array}{l}+0.002 \\
{ }_{-0.002}\end{array}$ & $\begin{array}{l}+0.019 \\
-0.017\end{array}$ & $\begin{array}{l}+0.016 \\
-0.014\end{array}$ & 0.081 & \pm 0.010 & \pm 0.001 & $\begin{array}{l}+0.001 \\
{ }_{-0.002}\end{array}$ & & \\
\hline $3.5-5.0$ & 0.254 & \pm 0.013 & \pm 0.001 & $\begin{array}{l}+0.002 \\
{ }_{-0.002}\end{array}$ & $\begin{array}{l}+0.034 \\
-0.030\end{array}$ & $\begin{array}{l}+0.030 \\
{ }_{-0.026}\end{array}$ & 0.128 & \pm 0.015 & \pm 0.000 & ${ }_{-0.003}^{+0.002}$ & ${ }_{-0.027}^{+0.025}$ & $\begin{array}{l}+0.031 \\
-0.028\end{array}$ \\
\hline
\end{tabular}


Table 8.8: Measured kaon fraction $\left(f_{K}\right)$ around prompt $D_{s}^{ \pm}$and prompt $D^{ \pm}$mesons in ranges of the invariant mass of the D candidate and the maximum- $p_{T}$ track found in the cone $\left(m_{D K}\right)$, for the same sign charge combination. The first uncertainty is the statistical error (stat) followed by the systematic uncertainties due to sources such as: Effect of kaons decaying in flight (DIF), probability density functions used to separate prompt and secondary D components (PDF), calibration of Time-of-Flight and $d E / d x$ distributions for particle identification (calib) and mis-identification of particle species (mis-ID).

\begin{tabular}{|c|c|c|c|c|c|c|c|c|c|c|c|c|}
\hline \multirow[t]{2}{*}{$\begin{array}{c}m_{D K} \text { range } \\
\left(\mathrm{GeV} / \mathrm{c}^{2}\right) \\
\end{array}$} & \multicolumn{6}{|c|}{$\begin{array}{l}\text { Same sign kaon fraction } \\
\text { around prompt } D_{s}^{ \pm}\end{array}$} & \multicolumn{6}{|c|}{$\begin{array}{l}\text { Same sign kaon fraction } \\
\text { around prompt } D^{ \pm}\end{array}$} \\
\hline & $f_{K}$ & stat & DIF & PDF & calib & mis-ID & $f_{K}$ & stat & DIF & $\mathrm{PDF}$ & calib & mis-ID \\
\hline $2.2-2.5$ & 0.115 & \pm 0.046 & \pm 0.003 & ${ }_{-0.001}^{+0.001}$ & $\begin{array}{l}+0.037 \\
-0.038\end{array}$ & $\begin{array}{l}+0.038 \\
{ }_{-0.034}\end{array}$ & 0.154 & \pm 0.017 & \pm 0.001 & $\begin{array}{l}+0.003 \\
-0.003\end{array}$ & $\begin{array}{l}+0.035 \\
-0.038\end{array}$ & $\begin{array}{l}+0.039 \\
-0.034\end{array}$ \\
\hline $2.5-2.6$ & 0.164 & \pm 0.017 & \pm 0.003 & ${ }_{-0.001}^{+0.001}$ & $\begin{array}{l}+0.028 \\
-0.029\end{array}$ & ${ }_{-0.026}^{+0.028}$ & 0.139 & \pm 0.012 & \pm 0.001 & ${ }_{-0.002}^{+0.002}$ & $\begin{array}{l}+0.027 \\
-0.028\end{array}$ & \\
\hline $2.6-2.7$ & 0.155 & \pm 0.012 & \pm 0.000 & ${ }_{-0.001}^{+0.001}$ & $\begin{array}{l}+0.023 \\
-0.024\end{array}$ & $\begin{array}{l}+0.023 \\
-0.021\end{array}$ & 0.124 & \pm 0.011 & \pm 0.000 & $\begin{array}{l}+0.002 \\
-0.002\end{array}$ & $\begin{array}{l}+0.022 \\
-0.023\end{array}$ & \\
\hline $2.7-2.8$ & 0.121 & \pm 0.009 & \pm 0.001 & $\begin{array}{l}+0.001 \\
-0.001\end{array}$ & $\begin{array}{l}+0.018 \\
-0.019\end{array}$ & $\begin{array}{l}+0.019 \\
-0.017\end{array}$ & 0.118 & \pm 0.010 & \pm 0.001 & $\begin{array}{l}+0.002 \\
-0.002\end{array}$ & $\begin{array}{l}+0.018 \\
-0.019\end{array}$ & $\begin{array}{l}+0.019 \\
-0.017\end{array}$ \\
\hline 2.8-3.0 & 0.122 & \pm 0.006 & \pm 0.001 & $\begin{array}{l}+0.001 \\
-0.001\end{array}$ & $\begin{array}{l}+0.013 \\
{ }_{-0.014}\end{array}$ & $\begin{array}{l}+0.014 \\
{ }_{-0.013}\end{array}$ & 0.114 & \pm 0.007 & \pm 0.000 & $\begin{array}{l}+0.001 \\
{ }_{-0.002}\end{array}$ & $\begin{array}{l}{ }_{-0.014}^{+0.013} \\
\end{array}$ & $\begin{array}{l}+0.014 \\
{ }_{-0.013}\end{array}$ \\
\hline 3.0-3.2 & 0.116 & \pm 0.006 & \pm 0.001 & $\begin{array}{l}+0.001 \\
-0.001\end{array}$ & $\begin{array}{l}+0.012 \\
-0.012\end{array}$ & $\begin{array}{l}+0.012 \\
-0.011\end{array}$ & 0.128 & \pm 0.009 & \pm 0.001 & $\begin{array}{l}+0.001 \\
-0.001\end{array}$ & $\begin{array}{l}+0.011 \\
-0.012\end{array}$ & \\
\hline $3.2-3.5$ & 0.136 & \pm 0.008 & \pm 0.001 & $\begin{array}{l}+0.001 \\
-0.001\end{array}$ & $\begin{array}{l}+0.015 \\
-0.015\end{array}$ & ${ }_{-0.013}^{+0.014}$ & 0.138 & \pm 0.012 & \pm 0.001 & $\begin{array}{l}+0.001 \\
-0.002\end{array}$ & $\begin{array}{l}+0.014 \\
-0.015\end{array}$ & $\begin{array}{l}+0.014 \\
-0.013\end{array}$ \\
\hline $3.5-5.0$ & 0.190 & \pm 0.013 & \pm 0.001 & $\begin{array}{l}+0.001 \\
-0.001 \\
\end{array}$ & $\begin{array}{l}+0.029 \\
-0.030 \\
\end{array}$ & $\begin{array}{l}+0.031 \\
-0.028 \\
\end{array}$ & 0.199 & \pm 0.020 & \pm 0.001 & $\begin{array}{l}+0.002 \\
-0.003 \\
\end{array}$ & $\begin{array}{l}+0.028 \\
-0.030 \\
\end{array}$ & $\begin{array}{l}+0.032 \\
-0.028 \\
\end{array}$ \\
\hline
\end{tabular}


Table 8.9: Measured kaon fraction around prompt $D_{s}^{ \pm}$and prompt $D^{ \pm}$mesons in ranges of the rapidity difference between the $\mathrm{D}$ candidate and the maximum- $p_{T}$ track found in the cone along the direction of the $\mathrm{D}$ momentum axis $(\Delta y)$, for the opposite sign charge combination. The first uncertainty is statistical and the second is the combination of all the systematic uncertainties.

\begin{tabular}{|c|c|c|}
\hline$\Delta y$ range & $\begin{array}{c}\text { Opposite sign kaon fraction } \\
\text { around prompt } D_{s}^{ \pm}\end{array}$ & $\begin{array}{c}\text { Opposite sign kaon fraction } \\
\text { around prompt } D^{ \pm}\end{array}$ \\
\hline$(-6,-4)$ & $0.1470 \pm 0.0089_{-0.0083}^{+0.0086}$ & $0.0674 \pm 0.0071_{-0.0060}^{+0.0058}$ \\
\hline$(-4,-3.5)$ & $0.173 \pm 0.008_{-0.010}^{+0.010}$ & $0.0634 \pm 0.0063_{-0.0072}^{+0.0070}$ \\
\hline$(-3.5,-3)$ & $0.208 \pm 0.0077_{-0.011}^{+0.012}$ & $0.0715 \pm 0.0054_{-0.0090}^{+0.0087}$ \\
\hline$(-3,-2.5)$ & $0.276 \pm 0.007_{-0.015}^{+0.017}$ & $0.083 \pm 0.007_{-0.013}^{+0.012}$ \\
\hline$(-2.5,-2)$ & $0.318 \pm 0.0099_{-0.022}^{+0.024}$ & $0.064 \pm 0.008_{-0.019}^{+0.018}$ \\
\hline$(-2,-1.5)$ & $0.382 \pm 0.012_{-0.031}^{+0.034}$ & $0.067 \pm 0.011_{-0.027}^{+0.027}$ \\
\hline$(-1.5,-1)$ & $0.455 \pm 0.015_{-0.042}^{+0.047}$ & $0.031 \pm 0.014_{-0.040}^{+0.039}$ \\
\hline$(-1,0)$ & $0.603 \pm 0.020{ }_{-0.061}^{+0.069}$ & $0.062 \pm 0.015_{-0.061}^{+0.062}$ \\
\hline$(0,2)$ & $0.575 \pm 0.033_{-0.084}^{+0.100}$ & $0.060 \pm 0.026_{-0.085}^{+0.091}$ \\
\hline
\end{tabular}


Table 8.10: Measured kaon fraction around prompt $D_{s}^{ \pm}$and prompt $D^{ \pm}$mesons in ranges of the rapidity difference between the $\mathrm{D}$ candidate and the maximum- $p_{T}$ track found in the cone along the direction of the $\mathrm{D}$ momentum axis $(\Delta y)$, for the same sign charge combination. The first uncertainty is statistical and the second is the combination of all the systematic uncertainties.

\begin{tabular}{|c|c|c|}
\hline$\Delta y$ range & $\begin{array}{l}\text { Same sign kaon fraction } \\
\text { around prompt } D_{s}^{ \pm}\end{array}$ & $\begin{array}{l}\text { Same sign kaon fraction } \\
\text { around prompt } D^{ \pm}\end{array}$ \\
\hline$(-6,-4)$ & $0.0964 \pm 0.0084_{-0.0070}^{+0.0070}$ & $0.0968 \pm 0.00899_{-0.0069}^{+0.0069}$ \\
\hline$(-4,-3.5)$ & $0.1125 \pm 0.00799_{-0.0082}^{+0.0083}$ & $0.1048 \pm 0.00899_{-0.0082}^{+0.0082}$ \\
\hline$(-3.5,-3)$ & $0.118 \pm 0.007{ }_{-0.010}^{+0.010}$ & $0.116 \pm 0.008_{-0.010}^{+0.010}$ \\
\hline$(-3,-2.5)$ & $0.139 \pm 0.007_{-0.014}^{+0.015}$ & $0.136 \pm 0.0088_{-0.015}^{+0.014}$ \\
\hline$(-2.5,-2)$ & $0.166 \pm 0.0099_{-0.021}^{+0.021}$ & $0.157 \pm 0.010_{-0.021}^{+0.021}$ \\
\hline$(-2,-1.5)$ & $0.172 \pm 0.013_{-0.029}^{+0.030}$ & $0.176 \pm 0.014_{-0.030}^{+0.029}$ \\
\hline$(-1.5,-1)$ & $0.208 \pm 0.020_{-0.042}^{+0.042}$ & $0.182 \pm 0.020_{-0.042}^{+0.041}$ \\
\hline$(-1,0)$ & $0.166 \pm 0.014_{-0.064}^{+0.066}$ & $0.216 \pm 0.0266_{-0.064}^{+0.066}$ \\
\hline$(0,2)$ & $0.11 \pm 0.044_{-0.09}^{+0.10}$ & $0.18 \pm 0.04_{-0.09}^{+0.10}$ \\
\hline
\end{tabular}


Table 8.11: Measured kaon fraction $\left(f_{K}\right)$ around prompt $D_{s}^{ \pm}$and prompt $D^{ \pm}$mesons in ranges of the rapidity difference between the $\mathrm{D}$ candidate and the maximum- $p_{T}$ track found in the cone along the direction of the $\mathrm{D}$ momentum axis $(\Delta y)$, for the opposite sign charge combination. The first uncertainty is the statistical error (stat) followed by the systematic uncertainties due to sources such as: Effect of kaons decaying in flight (DIF), probability density functions used to separate prompt and secondary D components (PDF), calibration of Time-of-Flight and $d E / d x$ distributions for particle identification (calib) and mis-identification of particle species (mis-ID).

\begin{tabular}{|c|c|c|c|c|c|c|c|c|c|c|c|c|}
\hline \multirow[t]{2}{*}{$\Delta y$ range } & \multicolumn{6}{|c|}{$\begin{array}{l}\text { Opposite sign kaon fraction } \\
\text { around prompt } D_{s}^{ \pm}\end{array}$} & \multicolumn{6}{|c|}{$\begin{array}{l}\text { Opposite sign kaon fraction } \\
\text { around prompt } D^{ \pm}\end{array}$} \\
\hline & $f_{K}$ & stat & $\mathrm{DIF}$ & $\mathrm{PDF}$ & calib & mis-ID & $f_{K}$ & stat & $\mathrm{DIF}$ & $\mathrm{PDF}$ & calib & mis-ID \\
\hline$(-6,-4)$ & 0.1470 & \pm 0.0089 & \pm 0.0006 & $\begin{array}{l}+0.0010 \\
-0.0010\end{array}$ & $\begin{array}{l}+0.0055 \\
-0.0051\end{array}$ & $\begin{array}{l}+0.0065 \\
-0.0064\end{array}$ & 0.0674 & \pm 0.0071 & \pm 0.0003 & $\begin{array}{l}+0.0009 \\
-0.0013\end{array}$ & $\begin{array}{l}+0.0038 \\
-0.0040\end{array}$ & $\begin{array}{l}+0.0043 \\
-0.0042\end{array}$ \\
\hline$(-4,-3.5)$ & 0.1731 & \pm 0.0083 & \pm 0.0008 & $\begin{array}{l}+0.0011 \\
-0.0011\end{array}$ & $\begin{array}{l}+0.0070 \\
-0.0064\end{array}$ & $\begin{array}{l}+0.0072 \\
-0.0070\end{array}$ & 0.0634 & \pm 0.0063 & \pm 0.0004 & $\begin{array}{l}+0.0008 \\
-0.0013\end{array}$ & $\begin{array}{l}+0.0047 \\
-0.0051\end{array}$ & $\begin{array}{l}+0.0051 \\
-0.0049\end{array}$ \\
\hline$(-3.5,-3)$ & 0.2075 & \pm 0.0070 & \pm 0.0008 & $\begin{array}{l}+0.0012 \\
-0.0013\end{array}$ & $\begin{array}{l}+0.0089 \\
-0.0082\end{array}$ & $\begin{array}{l}+0.0082 \\
-0.0079\end{array}$ & 0.0715 & \pm 0.0054 & \pm 0.0003 & $\begin{array}{l}+0.0009 \\
-0.0012\end{array}$ & $\begin{array}{l}+0.0061 \\
-0.0066\end{array}$ & $\begin{array}{l}+0.0062 \\
-0.0059\end{array}$ \\
\hline$(-3,-2.5)$ & 0.276 & \pm 0.007 & \pm 0.001 & $\begin{array}{l}+0.001 \\
-0.002\end{array}$ & $\begin{array}{l}+0.013 \\
-0.012\end{array}$ & $\begin{array}{l}+0.011 \\
-0.010\end{array}$ & 0.0833 & \pm 0.0066 & \pm 0.0002 & $\begin{array}{l}+0.0010 \\
-0.0013\end{array}$ & $\begin{array}{l}+0.0087 \\
-0.0095\end{array}$ & $\begin{array}{l}+0.0087 \\
-0.0082\end{array}$ \\
\hline$(-2.5,-2)$ & 0.318 & \pm 0.009 & \pm 0.001 & $\begin{array}{l}+0.002 \\
-0.002\end{array}$ & $\begin{array}{l}+0.019 \\
-0.017\end{array}$ & $\begin{array}{l}+0.015 \\
-0.014\end{array}$ & 0.064 & \pm 0.008 & \pm 0.002 & $\begin{array}{l}+0.001 \\
-0.001\end{array}$ & $\begin{array}{l}+0.013 \\
-0.014\end{array}$ & $\begin{array}{l}+0.013 \\
-0.012\end{array}$ \\
\hline$(-2,-1.5)$ & 0.382 & \pm 0.012 & \pm 0.002 & $\begin{array}{l}+0.002 \\
-0.002\end{array}$ & $\begin{array}{l}+0.027 \\
-0.024\end{array}$ & $\begin{array}{l}+0.020 \\
-0.018\end{array}$ & 0.067 & \pm 0.011 & \pm 0.001 & $\begin{array}{l}+0.002 \\
-0.002\end{array}$ & $\begin{array}{l}+0.018 \\
-0.020\end{array}$ & $\begin{array}{l}+0.019 \\
-0.018\end{array}$ \\
\hline$(-1.5,-1)$ & 0.455 & \pm 0.015 & \pm 0.002 & $\begin{array}{l}+0.002 \\
-0.003\end{array}$ & $\begin{array}{l}+0.038 \\
-0.034\end{array}$ & $\begin{array}{l}+0.028 \\
-0.025\end{array}$ & 0.031 & \pm 0.014 & \pm 0.004 & $\begin{array}{l}+0.002 \\
-0.002\end{array}$ & $\begin{array}{l}+0.026 \\
-0.029\end{array}$ & $\begin{array}{l}+0.029 \\
-0.026\end{array}$ \\
\hline$(-1,0)$ & 0.603 & \pm 0.020 & \pm 0.004 & $\begin{array}{l}+0.003 \\
-0.003\end{array}$ & $\begin{array}{l}+0.053 \\
-0.047\end{array}$ & $\begin{array}{l}+0.045 \\
-0.039\end{array}$ & 0.062 & \pm 0.015 & \pm 0.003 & $\begin{array}{l}+0.003 \\
-0.002\end{array}$ & $\begin{array}{l}+0.038 \\
-0.042\end{array}$ & $\begin{array}{l}+0.049 \\
-0.044\end{array}$ \\
\hline$(0,2)$ & 0.575 & \pm 0.033 & \pm 0.007 & $\begin{array}{l}+0.003 \\
-0.003\end{array}$ & $\begin{array}{l}+0.064 \\
-0.055\end{array}$ & $\begin{array}{l}+0.076 \\
-0.064\end{array}$ & 0.060 & \pm 0.026 & \pm 0.003 & $\begin{array}{l}+0.006 \\
-0.004\end{array}$ & $\begin{array}{l}+0.050 \\
-0.052\end{array}$ & $\begin{array}{l}+0.076 \\
-0.067\end{array}$ \\
\hline
\end{tabular}


Table 8.12: Measured kaon fraction $\left(f_{K}\right)$ around prompt $D_{s}^{ \pm}$and prompt $D^{ \pm}$mesons in ranges of the rapidity difference between the $\mathrm{D}$ candidate and the maximum- $p_{T}$ track found in the cone along the direction of the $\mathrm{D}$ momentum axis $(\Delta y)$, for the same sign charge combination. The first uncertainty is the statistical error (stat) followed by the systematic uncertainties due to sources such as: Effect of kaons decaying in flight (DIF), probability density functions used to separate prompt and secondary D components (PDF), calibration of Time-of-Flight and $d E / d x$ distributions for particle identification (calib) and mis-identification of particle species (mis-ID).

\begin{tabular}{|c|c|c|c|c|c|c|c|c|c|c|c|c|}
\hline \multirow[t]{2}{*}{$\Delta y$ range } & \multicolumn{6}{|c|}{$\begin{array}{l}\text { Same sign kaon fraction } \\
\text { around prompt } D_{s}^{ \pm}\end{array}$} & \multicolumn{6}{|c|}{$\begin{array}{l}\text { Same sign kaon fraction } \\
\text { around prompt } D^{ \pm}\end{array}$} \\
\hline & $f_{K}$ & stat & DIF & $\mathrm{PDF}$ & calib & mis-ID & $f_{K}$ & stat & DIF & $\mathrm{PDF}$ & calib & mis-ID \\
\hline$(-6,-4)$ & 0.0964 & \pm 0.0084 & \pm 0.0002 & $\begin{array}{l}+0.0003 \\
-0.0003\end{array}$ & $\begin{array}{l}+0.0046 \\
-0.0047\end{array}$ & $\begin{array}{l}+0.0052 \\
-0.0051\end{array}$ & 0.0968 & \pm 0.0089 & \pm 0.0005 & $\begin{array}{l}+0.0009 \\
-0.0010\end{array}$ & $\begin{array}{l}+0.0045 \\
-0.0046\end{array}$ & $\begin{array}{l}+0.0051 \\
-0.0049\end{array}$ \\
\hline$(-4,-3.5)$ & 0.1125 & \pm 0.0079 & \pm 0.0003 & $\begin{array}{l}+0.0004 \\
-0.0005\end{array}$ & $\begin{array}{l}+0.0058 \\
-0.0059\end{array}$ & $\begin{array}{l}+0.0059 \\
-0.0057\end{array}$ & 0.1048 & \pm 0.0089 & \pm 0.0006 & $\begin{array}{l}+0.0010 \\
-0.0011\end{array}$ & $\begin{array}{l}+0.0056 \\
-0.0058\end{array}$ & $\begin{array}{l}+0.0059 \\
-0.0056\end{array}$ \\
\hline$(-3.5,-3)$ & 0.1181 & \pm 0.0066 & \pm 0.0007 & $\begin{array}{l}+0.0005 \\
-0.0005\end{array}$ & $\begin{array}{l}+0.0076 \\
-0.0077\end{array}$ & $\begin{array}{l}+0.0071 \\
-0.0068\end{array}$ & 0.1156 & \pm 0.0079 & \pm 0.0007 & $\begin{array}{l}+0.0011 \\
-0.0013\end{array}$ & $\begin{array}{l}+0.0073 \\
-0.0077\end{array}$ & $\begin{array}{l}+0.0071 \\
-0.0067\end{array}$ \\
\hline$(-3,-2.5)$ & 0.139 & \pm 0.007 & \pm 0.001 & $\begin{array}{l}+0.001 \\
-0.001\end{array}$ & $\begin{array}{l}+0.011 \\
-0.011\end{array}$ & $\begin{array}{l}+0.010 \\
-0.009\end{array}$ & 0.136 & \pm 0.008 & \pm 0.001 & $\begin{array}{l}+0.001 \\
-0.002\end{array}$ & $\begin{array}{l}+0.011 \\
-0.011\end{array}$ & $\begin{array}{l}+0.010 \\
-0.009\end{array}$ \\
\hline$(-2.5,-2)$ & 0.166 & \pm 0.009 & \pm 0.001 & $\begin{array}{l}+0.001 \\
-0.001\end{array}$ & $\begin{array}{l}+0.016 \\
-0.016\end{array}$ & $\begin{array}{l}+0.014 \\
-0.013\end{array}$ & 0.157 & \pm 0.010 & \pm 0.001 & $\begin{array}{l}+0.002 \\
-0.002\end{array}$ & $\begin{array}{l}+0.015 \\
-0.016\end{array}$ & $\begin{array}{l}+0.014 \\
-0.013\end{array}$ \\
\hline$(-2,-1.5)$ & 0.172 & \pm 0.013 & \pm 0.001 & $\begin{array}{l}+0.001 \\
-0.001\end{array}$ & $\begin{array}{l}+0.022 \\
-0.023\end{array}$ & $\begin{array}{l}+0.020 \\
-0.018\end{array}$ & 0.176 & \pm 0.014 & \pm 0.003 & $\begin{array}{l}+0.002 \\
-0.002\end{array}$ & $\begin{array}{l}+0.022 \\
-0.023\end{array}$ & $\begin{array}{l}+0.020 \\
-0.018\end{array}$ \\
\hline$(-1.5,-1)$ & 0.208 & \pm 0.020 & \pm 0.001 & $\begin{array}{l}+0.001 \\
-0.001\end{array}$ & $\begin{array}{l}+0.031 \\
-0.033\end{array}$ & $\begin{array}{l}+0.028 \\
-0.026\end{array}$ & 0.182 & \pm 0.020 & \pm 0.001 & $\begin{array}{l}+0.002 \\
-0.003\end{array}$ & $\begin{array}{l}+0.030 \\
-0.033\end{array}$ & $\begin{array}{l}+0.028 \\
-0.026\end{array}$ \\
\hline$(-1,0)$ & 0.166 & \pm 0.014 & \pm 0.000 & $\begin{array}{l}+0.001 \\
-0.001\end{array}$ & $\begin{array}{l}+0.046 \\
-0.048\end{array}$ & $\begin{array}{l}+0.048 \\
-0.043\end{array}$ & 0.216 & \pm 0.026 & \pm 0.005 & $\begin{array}{l}+0.003 \\
-0.003\end{array}$ & $\begin{array}{l}+0.044 \\
-0.047\end{array}$ & $\begin{array}{l}+0.049 \\
-0.043\end{array}$ \\
\hline$(0,2)$ & 0.110 & \pm 0.035 & \pm 0.006 & $\begin{array}{l}+0.002 \\
-0.002 \\
\end{array}$ & $\begin{array}{l}+0.059 \\
-0.059 \\
\end{array}$ & $\begin{array}{l}+0.084 \\
-0.073 \\
\end{array}$ & 0.181 & \pm 0.039 & \pm 0.009 & $\begin{array}{l}+0.004 \\
-0.005 \\
\end{array}$ & $\begin{array}{l}+0.057 \\
-0.058 \\
\end{array}$ & $\begin{array}{l}+0.087 \\
-0.073 \\
\end{array}$ \\
\hline
\end{tabular}




\subsection{Cross check using displaced tracks around secondary $D_{s}^{ \pm}$}

As described in the previous sections, the measurement of kaon fraction around the prompt $D_{s}^{ \pm} / D^{ \pm}$components is done using a multidimensional likelihood fitting procedure. We conduct a cross check of this procedure using a sample of displaced tracks around secondary $D_{s}^{ \pm}$, and compare it to the kaon fraction expected in a sample of tracks that can be modeled with reasonable accuracy.

This study involves comparing the measured fraction of kaons produced in $B$ decays with predictions of the PYTHIA event generator, using a sample of tracks with impact parameter in the range $200 \mu \mathrm{m}<d_{0}<2 \mathrm{~mm}$, found in a cone of radius $\Delta R=0.7$ around the reconstructed $K K \pi$ candidates. This impact parameter selection is the opposite of the criteria used for tracks in the charm fragmentation study in which we require $d_{0}<200 \mu \mathrm{m}$.

In this cross check, we measure the kaon fraction in the displaced track sample around the secondary $D_{s}^{ \pm}$and $D^{ \pm}$components in data. However, we can conduct the cross check more reliably using the secondary $D_{s}^{ \pm}$component, which is about $25 \%$ of the total $D_{s}^{ \pm}$component. In addition, we do not split the sample of displaced tracks into opposite-sign and same-sign charge tracks since we are not particularly interested in studying charge correlations in the displaced track sample.

We compare the measured kaon fraction around secondary $D_{s}^{ \pm}$component obtained from the fitting procedure with the kaon fraction around secondary $D_{s}^{ \pm}$mesons found in a generic $b \bar{b}$ sample generated using the PYTHIA event generator. The kaon fraction measured in ranges of $p_{T}$ is listed in the Table 8.13. The measured fraction in the PYTHIA sample is also shown in the table. Figure 8.4 shows a graph of the distribution of the measured kaon fraction as a function of transverse momentum.

The results of the cross check indicate that the measured kaon fraction is in reasonable agreement with PYTHIA predictions. This implies that we can interpret the measured kaon fraction around secondary $D_{s}^{ \pm}$using the Monte Carlo simulations of $\mathrm{B}$ decay products. Hence, we can conclude that the kaon fractions, extracted using 
our measurement technique, in other samples such as the sample used in the charm fragmentation study, are determined with similar or better accuracy.

Table 8.13: Measured kaon fraction in a sample of displaced tracks found in a cone around secondary $D_{s}^{ \pm}$mesons. The table also shows predictions of the PYTHIA event generator.

\begin{tabular}{|c|c|c|}
\hline $\begin{array}{c}p_{T} \text { range } \\
(\mathrm{GeV} / \mathrm{c})\end{array}$ & $\begin{array}{c}\text { Kaon fraction } \\
\text { around secondary } D_{s}^{ \pm} \\
\text {(Data) }\end{array}$ & $\begin{array}{c}\text { Kaon fraction } \\
\text { around secondary } D_{s}^{ \pm} \\
\text {(PYTHIA) }\end{array}$ \\
\hline $0.4-0.6$ & $0.0440_{-0.0251}^{+0.0247}$ & $0.0628 \pm 0.0013$ \\
\hline $0.6-0.8$ & $0.1177_{-0.0246}^{+0.0246}$ & $0.1228 \pm 0.0020$ \\
\hline $0.8-1.0$ & $0.1611_{-0.0287}^{+0.0287}$ & $0.1908 \pm 0.0027$ \\
\hline $1.0-1.4$ & $0.1783_{-0.0382}^{+0.0377}$ & $0.2336 \pm 0.0024$ \\
\hline $1.4-2.0$ & $0.1770_{-0.0438}^{+0.0441}$ & $0.2292 \pm 0.0024$ \\
\hline $2.0-6.0$ & $0.2897_{-0.1179}^{+0.1313}$ & $0.2016 \pm 0.0018$ \\
\hline
\end{tabular}




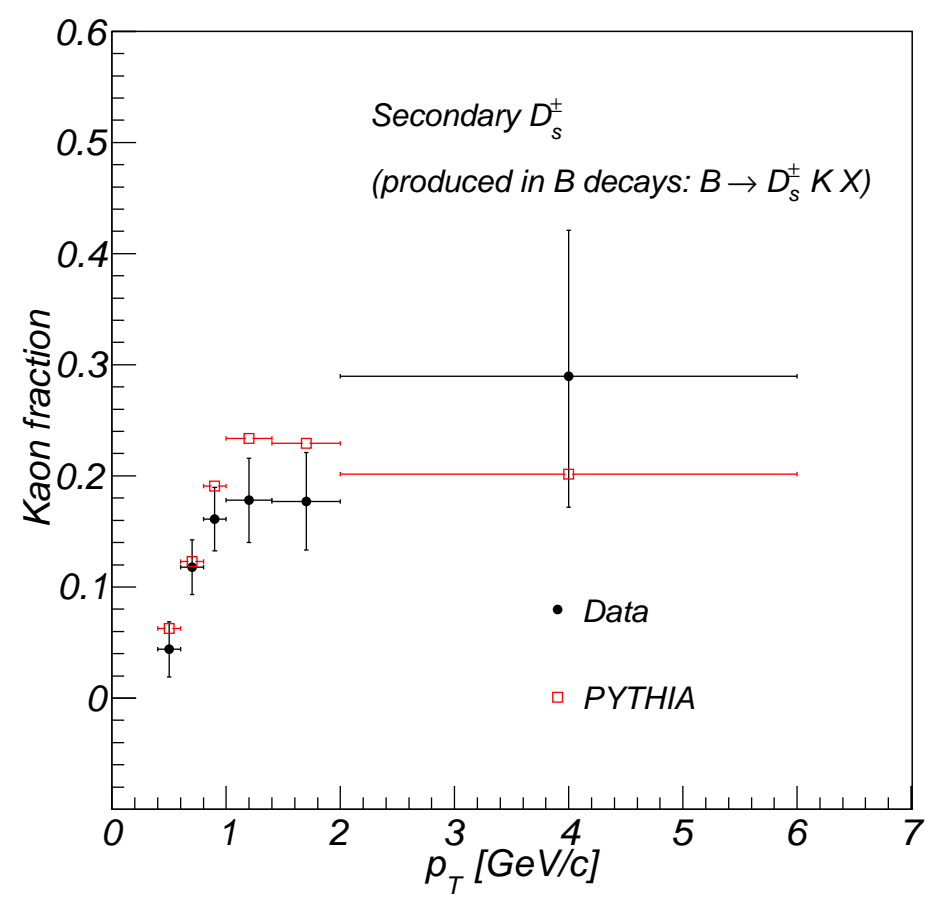

Figure 8.4.: Distribution of kaon fraction measured around secondary $D_{s}^{ \pm}$mesons as a function of the transverse momentum of the maximum- $p_{T}$ displaced track found in the cone. 


\section{SUMMARY}

We conducted an analysis to study some aspects of the non-perturbative quark fragmentation phenomenon by measuring the distribution of kaon fraction around prompt $D_{s}^{ \pm} / D^{ \pm}$mesons produced via the hadronization of charm quarks. The results in data indicate that the in the opposite sign $D_{(s)}^{ \pm} K^{\mp}$ charge combination, kaon production around prompt $D_{s}^{ \pm}$is enhanced compared to production around prompt $D^{ \pm}$.

In the same sign $D_{(s)}^{ \pm} K^{ \pm}$charge combination, kaon production around prompt $D_{s}^{ \pm}$and prompt $D^{ \pm}$is similar. The enhanced production of oppositely charged kaons around prompt $D_{s}^{ \pm}$mesons is a feature of the phenomenological models used to describe the fragmentation process in the PYTHIA and HERWIG Monte Carlo event generators.

We compared various kinematic distribution of the measured kaon fraction around prompt $D_{s}^{ \pm} / D^{ \pm}$mesons with predictions of the string fragmentation model used in the PYTHIA event generator and the cluster fragmentation model used in the HERWIG event generator. The results of the comparative study indicate that the $p_{T}$ distributions for early fragmentation kaons produced around prompt $D_{s}^{ \pm}$are in better qualitative agreement with predictions of fragmentation models compared to same sign kaons that are produced in later fragmentation branches, for which the models underestimate the fraction of kaons.

Conversely, the distribution of the measured kaon fraction as a function of the $D_{s}^{ \pm}$kaon invariant mass and $D_{s}^{ \pm}$-kaon rapidity-difference indicate that the fragmentation models overestimate the fraction of kaons produced in early stages of the fragmentation process, compared to the fraction of same sign kaons that are produced in later branches, for which the predictions of the models are in better qualitative agreement with the distribution in data. 
The charm fragmentation study quantifies enhanced kaon production around heavy mesons containing a strange quark, which has been observed previously in the B system. However, this is the first study to directly measure the charge correlation between kaons and heavy strange mesons, which formed the basis for the same side kaon tagging technique used in the $B_{s}^{0}$ mixing analysis to infer the initial flavor of the $b$ quark in the $B_{s}^{0}$ mixing analysis. By specifically measuring the kinematic distributions of opposite sign kaons produced in association with $D_{s}^{ \pm}$mesons, we probe the second branching in the fragmentation process that has never been studied before. 
LIST OF REFERENCES 


\section{LIST OF REFERENCES}

[1] K. Nakamura et al. (Particle Data Group), J. Phys. G 37, 075021 (2010).

[2] L.B. Okun, Particle Physics: The Quest for the Substance of Substance, Harwood Academic Publishers, 1985.

[3] D. H. Perkins Introduction to High Energy Physics, Cambridge University Press, 2000.

[4] V. Barger and R. Phillips, Collider Physics, Addison-Wesley Publishing Company, 1997.

[5] G. Aad et al (The ATLAS collaboration), Phys. Lett. B 710, 49-66 (2012).

[6] S. Chatrchyan et al (The CMS collaboration), Phys. Lett. B 710, 26-48 (2012).

[7] L. L. Chau and W. Y. Keung, Phys. Rev. Lett. 53, 1802 (1984).

[8] L. Wolfenstein, Phys. Rev. Lett. 51, 1945 (1983).

[9] F. Halzen and A. Martin, Quarks \&3 Leptons: An Introductory Course in Modern Particle Physics, John Wiley \& Sons Inc, 1984.

[10] R. D. Field and R. P. Feynman, Nucl. Phys. B 136, 1 (1978).

[11] R. D. Field, Applications of Perturbative QCD, Addison-Wesley Publishing Company, The Advanced Book Program, 1989.

[12] B. Andersson et al, Phys. Rev. Lett. 97, 31 (1983).

[13] T. Sjöstrand, Nuclear Physics B 248, 469 (1984).

[14] C. Peterson et al, Phys. Rev. D 27, 105 (1983).

[15] D. Amati and G. Veneziano, Phys. Lett. B 83, 87 (1979).

[16] http://www-bdnew.fnal.gov/operations/rookie_books/rbooks.html

[17] R. C. Fernow, Introduction to experimental particle physics, Cambridge University Press, 1986.

[18] R. Moore, Tevatron Run II series at the Enrico Fermi Laboratory (USA), Atomic Energy, Vol. 93, No. 6, pp. 416-420, December 2002.

[19] The CDF Collaboration, The CDF II Detector, Technical Design Report FERMILAB-Pub-96/390-E.

[20] T. Affolder et al, CDF Central Outer Tracker Nuclear Instruments and Methods in Physics Research A, Volume 526, Pages 249-299, July 2004. 
[21] D. Acosta et al, A Time-of-Flight detector in CDF-II Nuclear Instruments and Methods in Physics Research A, Volume 518, Pages 605-608, February 2004.

[22] E. J. Thomson et al, Online Track Processor for the CDF Upgrade IEEE Transactions on Nuclear Science, Volume 49, No. 3, Page 1063, June 2002.

[23] B. Ashmanskas et al., Nucl. Instrum. Methods Phys. Res. A 518, 532 (2004);

[24] A. Belloni et al, Event Builder and Level 3 at the CDF experiment Nuclear Instruments and Methods in Physics Research A, Volume 518, Pages 522-524, February 2004.

[25] A. Belloni et al, Multibody Trigger Paths in the Two Track Trigger Data, CDF Note 6526, July 28, 2003.

[26] D. Acosta et al (The CDF collaboration) Phys. Rev. Lett. 95, 031801 (2005).

[27] T. Aaltonen et al (The CDF collaboration) Phys. Rev. Lett. 103, 191802 (2009).

[28] T. Aaltonen et al (The CDF collaboration) Phys. Rev. D81, 031105 (2010).

[29] S. Cabrera et al, The CDF-II Time-of-Flight Detector, CDF Note 6258, February 3 (2003).

[30] M. Jones, Initial Performance Studies of the CDF-II Time-of-Flight Detector, CDF Note 5930, May 22 (2002).

[31] N. Ranjan and M. Jones, Parameterization of the Tails in TOF Residual Distribution Using Soft Pions from D* decays, CDF Note 10209, July 2 (2010).

[32] C. Grupen and B. Shwartz, Particle Detectors, Cambridge University Press, 2008.

[33] N. Ranjan and M. Jones, COT Energy Loss Calibration for Low $p_{T}$ Unbiased Tracks, CDF Note 10272, August 24, 2010.

[34] D. Usynin, M. Jones and J. Kroll, Study of Charged Particle Species Produced in Association with $B^{0}, B^{+}$and $B_{s}$ Mesons, CDF Note 7810, October 15, 2005.

[35] C. Chen, R. Oldeman and J. Kroll, A Method to Measure the Direct and Secondary Charm Fraction in Two Track Data, CDF Note 5996, July 1, 2002.

[36] D. Acosta et al., The CDF Collaboration, Phys. Rev. Lett. 91, 241804 (2003).

[37] N. Ranjan and M. Jones, Parameterization of Secondary $D_{s}^{ \pm} / D^{ \pm}$Impact Parameter Distributions, CDF Note 9749, January 29, 2010.

[38] T. Sjostrand, S. Mrenna, P. Skands, A Brief Introduction to PYTHIA 8.1, LU TP 07-28, October 2007.

[39] G. Corcella, I.G. Knowles, G. Marchesini, S. Moretti, K. Odagiri, P. Richardson, M.H. Seymour and B.R. Webber, HERWIG 6.5 Release Note, hep-ph/0210213.

[40] N. Ranjan and M. Jones, A Study of Quark Fragmentation using Kaons Produced in Association with $D_{s}^{+} / D^{+}$Mesons, CDF Note 10662, September 28, 2011. 
[41] D. Usynin, M. Jones, J. Kroll, Study of Charged Particle Species Produced in Association with $B^{0}, B^{-}$and $B_{s}$ Mesons, CDF Note 8137, April 13, 2006.

[42] M. Casarsa, P. Catastini, M.A. Ciocci, G. Punzi, L. Ristori and A.M. Zanetti, A Method to Disentangle Distributions of Kinematical Variables of Different Particle Species, CDF Note 8453, August 11, 2006.

[43] M. Casarsa and P. Catastini, Comparison of the Kinematics of each Particle Species Produced around B Meson in Pythia and Data, CDF Note 8856, June 11, 2007.

[44] http://www-cdf.fnal.gov/internal/physics/bottom/bmontecarlo/instructions_2009/

[45] P. Squillacioti, et al, Particle identitication by combining TOF and $d E / d x$ information, CDF Note 7488, March 22, 2005. 
VITA 


\section{VITA}

Niharika Ranjan Singh was a Ph.D. candidate in the Department of Physics at Purdue University, West Lafayette, Indiana. Her research interests included experimental particle physics, nuclear physics and astrophysics. She joined the Physics department in Fall 2003 and was a teaching assistant until Spring 2005.

She started her doctoral thesis work under the guidance of Prof. Matthew Jones in Spring 2005. Niharika was affiliated with the CDF collaboration at Fermilab during the course of her doctoral degree. She was based at Fermilab for two years, from Summer 2005 to Summer 2007. Niharika graduated from Purdue university in May 2012 with a Ph.D. in Experimental Particle Physics.

Prior to joining Purdue University in 2003, Niharika obtained her Master of Science (M.Sc.) in Physics from Indian Institute of Technology Madras, Chennai, India. During the summer of 2002, she worked as a summer intern at the Saha Institute of Nuclear Physics, Kolkata, India. She completed her Bachelor's degree in Science (B.Sc.) in 2001 from University of Rajasthan, Jaipur, India in Physics, Chemistry and Mathematics. 